JOURNAL OF THE

AMERICAN MATHEMATICAL SOCIETY

Volume 13, Number 2, Pages 243-294

S 0894-0347(00)00325-8

Article electronically published on January 31, 2000

\title{
INTERPOLATING HEREDITARILY INDECOMPOSABLE BANACH SPACES
}

\author{
S. A. ARGYROS AND V. FELOUZIS
}

\section{INTRODUCTION}

A Banach space $X$ is said to be Hereditarily Indecomposable (H.I.) if for any pair of closed subspaces $Y, Z$ of $X$ with $Y \cap Z=\{0\}, Y+Z$ is not a closed subspace. (Throughout this section by the term "subspace" we mean a closed infinite-dimensional subspace of $X$.) The H.I. spaces form a new and, as we believe, fundamental class of Banach spaces. The celebrated example of a Banach space with no unconditional basic sequence, due to W. Gowers and B. Maurey ([GM]), is the first construction of a H.I. space. It is easily seen that every H.I. space does not contain any unconditional basic sequence. Actually, the concept of H.I. spaces came after W. Johnson's observation that this was a property of the Gowers-Maurey example. To describe even further the peculiar structure of a H.I. space, we recall an alternative definition of such a space. A Banach space $X$ is a H.I. space if and only if for every pair of subspaces $Y, Z$ and $\varepsilon>0$ there exist $y \in Y, z \in Z$ with $\|y\|=\|z\|=1$ and $\|y-z\|<\varepsilon$. Thus, H.I. spaces are structurally irrelevant to classical Banach spaces, in particular to Hilbert spaces. Other constructions of H.I. spaces already exist. We mention Argyros and Deliyanni's construction of H.I. spaces which are asymptotic $\ell^{1}$ spaces ([AD2]), V. Ferenczi's example of a uniformly convex H.I. space (F2]) and H.I. modified asymptotic $\ell^{1}$ spaces contained in ADKM. Other examples of Banach spaces which are H.I. or which have a H.I. subspace are given in [G1, [H], OS1].

The construction of such a space requires several steps and it uses two fundamental ideas. The first is Tsirelson's recursive definition of saturated norms ([Ts]) and the second is Maurey-Rosenthal's construction of weakly null sequences without unconditional basic subsequences $([\mathrm{MR}]$ ). An important ingredient in the GowersMaurey construction is the Schlumprecht space. This is a Tsirelson type Banach space which is arbitrarily distortable and has been used in the solution of important problems. Thus beyond its use in the constructions of H.I. spaces it plays a central role in the solution of the distortion problem for Hilbert spaces ([OS]). The essential difference between Schlumprecht and Tsirelson spaces became more transparent in [AD2] where the mixed Tsirelson spaces were introduced. It is natural to expect that H.I. spaces share special and interesting properties not found in the previously known Banach spaces. Indeed, the following theorem is proven in

Received by the editors April 14, 1998 and, in revised form, June 8, 1999.

2000 Mathematics Subject Classification. Primary 46B20, 46B70; Secondary 46B03, 52A07, $03 \mathrm{E} 05$.

Key words and phrases. Interpolation methods, hereditarily indecomposable spaces, thin convex sets, Schreier families, summability methods.

(C)2000 American Mathematical Society 
GM]: Every bounded linear operator from a complex H.I. space $X$ to itself is of the form $\lambda I+S$, where $I$ denotes the identity operator and $S$ is a strictly singular operator. The same property of $\mathcal{L}(X, X)$ remains valid for certain real H.I. spaces like Gowers-Maurey space and Argyros-Deliyanni space. We recall that an operator is strictly singular if its restriction to any subspace is not an isomorphism. As a consequence of this theorem, every H.I. space (real or complex) is not isomorphic to any of its proper subspaces. Thus any H.I. space settles in the negative the famous Hyperplane Problem. Later on, Gowers proved a new dichotomy for Banach spaces $([\mathrm{G}])$. His theorem states that every Banach space either contains an unconditional basic sequence or it contains a H.I. subspace. We will use this result to prove our dichotomy related to quotients of H.I. spaces. Let us mention that Gowers' dichotomy leads to a solution of the homogeneous space problem. Also N. Tomczak-Jaegermann solved the distortion problem for H.I. spaces by showing that every H.I. space is arbitrarily distortable ([To]).

In the present paper we demonstrate that in spite of the fact that the structure of H.I. spaces is irrelevant to that of spaces with an unconditional basis, still, there are ways to connect these two classes. Thus we show that the class of Banach spaces which are quotients of H.I. spaces is extensive and, further on, large classes of operators between Banach spaces are factorized through H.I. spaces.

The structure of the quotients of H.I. spaces has also been studied by Ferenczi in [F1], who showed that every quotient of the Gowers-Maurey space is a H.I. space and, at the same time, there exists a quotient of a H.I. space which is not a H.I. space. The answer to the general question of whether every separable Banach space is a quotient of a H.I. space is negative, due to the lifting property of $\ell^{1}(\mathbb{N})$. Indeed, as is well known, every Banach space that has $\ell^{1}(\mathbb{N})$ as a quotient has a complemented subspace isomorphic to $\ell^{1}(\mathbb{N})$ and hence is not a H.I. space. The following result shows that $\ell^{1}(\mathbb{N})$ is somehow the only exception. To be more precise we prove the following dichotomy:

Theorem 1.1. Every Banach space $X$ either contains a subspace isomorphic to $\ell^{1}(\mathbb{N})$ or it has a subspace which is a quotient of a H.I. Banach space.

Further on, every member in the family of the so-called, classical Banach spaces not containing $\ell^{1}(\mathbb{N})$ is actually a quotient of a H.I. space. For example, we show that separable Hilbert spaces, or even $L^{p}(\lambda), 1<p<\infty, c_{0}(\mathbb{N})$, are quotients of H.I. Banach spaces. Let us observe that, by standard duality arguments, Theorem 1.1 gives that the dual of a H.I. space could contain isomorphically $L^{p}(\lambda), 1<p<$ $\infty$, or $\ell^{1}(\mathbb{N})$ and thus, in general, is not a H.I. space.

The proof of these results is rather extended and based upon the following two ingredients. The first is a general construction of H.I. interpolation spaces and the second is the geometric concept of a thin or an a-thin norming set. We will be more detailed about these shortly. We now give the definition of an a-thin set, suggested to us by B. Maurey, in order to present the factorization results of this paper.

For a null sequence $\mathbf{a}=\left(a_{n}\right)_{n \in \mathbb{N}}$ of positive real numbers and a bounded convex symmetric subset $W$ of a Banach space $X$ we say that $W$ is an a-thin set if the sequence $\left(\|\|_{n}\right)_{n}$ of equivalent norms on $X$, defined by the Minkowski gauges of the sets $2^{n} W+a_{n} B_{X}$, is not uniformly bounded on the unit ball of every subspace of $X$.

The second result of this paper concerns factorization of operators and it is the following: 
Theorem 1.2. Let $T: X \rightarrow Y$ be a bounded linear operator between Banach spaces such that $T\left[B_{X}\right]$ is an $\mathbf{a}$-thin set. Then there exist a H.I. space $Z$ and bounded linear operators $F_{1}: X \rightarrow Z, F_{2}: Z \rightarrow Y$ such that $T=F_{2} \circ F_{1}$ (i.e. $T$ is factorized through a H.I. space).

As a consequence of this theorem we show that every $T \in \mathcal{L}\left(\ell^{p}, \ell^{q}\right)$, where $p \neq q, p, q \in[1, \infty)$, is factorized through a H.I. space. Additionally, the identity map $I: L^{\infty}(\lambda) \rightarrow L^{1}(\lambda)$ is also factorized through a H.I. space and so is every strictly singular operator $T \in \mathcal{L}\left(\ell^{p}, \ell^{p}\right)$.

The next result of the paper refers to the structure of the H.I. spaces. As we mentioned in the beginning, Gowers and Maurey have shown that for $X$ a complex H.I. space, every $T$ in $\mathcal{L}(X, X)$ is of the form $\lambda I+S$ where $S$ is strictly singular. Further the same holds for some of the existing examples like GM], [AD2. It is not known whether a Banach space $X$ such that every $T$ in $\mathcal{L}(X, X)$ is of the form $\lambda I+K$, where $K$ is a compact operator, does exist. In general, the construction of strictly singular but not compact operators on a H.I. space does not seem easy. What is already known is a construction, due to T. Gowers (G2]), of an operator $T$ from a subspace $Y$ of Gowers-Maurey space $X$ to the whole space which is strictly singular and not compact. It is shown here that H.I. spaces with many strictly singular non-compact operators do exist. More precisely we have the following result:

Theorem 1.3. There exists a H.I. space $X$ with the property that for every subspace $Y$ of $X$ there exists a strictly singular non-compact operator $T \in \mathcal{L}(X, X)$ with the range of $T$ contained in $Y$.

The last result of the paper that we would like to mention concerns $\ell^{p}$-saturated Banach spaces. We recall that a Banach space $X$ is said to be $\ell^{p}$-saturated, for $p \in[1, \infty)$, if every subspace of $X$ contains a further subspace which is isomorphic to $\ell^{p}$. (The class of $c_{0}$-saturated Banach spaces is similarly defined.)

Theorem 1.4. Every reflexive Banach space $X$ with an unconditional basis contains a subspace $Y$ with the following property: for every $p \in[1, \infty)($ resp. $p=0)$ there exists an $\ell_{p}$-saturated (resp. $c_{0}$-saturated) space $Z$ which has $Y$ as a quotient.

We also show that when $X$ is some $L^{q}(\lambda), 1<q<\infty$, the subspace $Y$ coincides with the whole space. Probably, the most interesting case of the above theorem is that of the quotients of $c_{0}$-saturated spaces and we mention that D.H. Leung ([Le] has shown that separable Hilbert spaces are quotients of $c_{0}$-saturated spaces. Our methods are different from his.

We now move on to describe how the paper is organized and also to explain briefly the basic ideas of the proofs of the theorems mentioned above.

As the title of the paper indicates, the general scheme that we follow is interpolation methods. It is convenient for us to use the Davis, Figiel, Johnson and Pelczynski method ([DFJP $)$ which we extend for cases where the external norm is not necessarily unconditional. Thus in the second section we introduce the $d$ product norm of the space $\Omega_{00}=\left(\prod_{n=1}^{\infty} X_{n}\right)_{00}$, where $\left(X_{n}\right)_{n}$ is a sequence of Banach spaces. Then for a sequence $\left(\|\|_{n}\right)_{n}$ of equivalent norms on a given Banach space $X$ and a $d$-product norm on $\Omega_{00}=\left(\prod_{n=1}^{\infty}\left(X,\|\|_{n}\right)\right)_{00}$ we consider the diagonal space 
$\Delta \tilde{X}$, in the completion $\tilde{X}$ of $\Omega_{00}$, consisting of the vectors of the form $\bar{x}=(x, x, \ldots)$ such that $x \in X$ and $\bar{x}$ belongs to $\tilde{X}$. The spaces $\Delta \tilde{X}$ are the interpolation spaces that we are going to use.

In the third section we define and study the key notions of thin and a-thin sets. The concept of thin sets was introduced in Neidinger's Ph.D. thesis $([\mathbb{N}])$ and it is defined as follows.

A convex bounded symmetric set $W$ in a space $X$ is called a thin set if for every subspace $Z$ of $X$ there exists $\varepsilon>0$ such that for every real $\lambda$ the set $B_{Z}$ is not contained in the set $\lambda W+\varepsilon B_{X}$. In a previous version of the paper we exclusively used the notion of a thin set. Then B. Maurey made the important observation that everything works if we use the concept of an a-thin set, introduced above, instead of a thin set. To explain the difference between these two notions we notice that if $W$ is a thin set, then it is an a-thin set for every null sequence $\mathbf{a}=\left(a_{n}\right)_{n}$ of positive real numbers. Let us point out that in most of the results, concerning thinness of sets, we are only able to establish a-thinness. The free choice of the sequence $\mathbf{a}$ is an essential advantage when we are dealing with such problems. Further on, B. Maurey showed us how to prove that $B_{L^{\infty}(\lambda)}$ is an a-thin subset of $L^{1}(\lambda)$ for an appropriate sequence $\mathbf{a}$. Notice that Rosenthal had observed that $B_{L^{\infty}(\lambda)}$ is not a thin subset of $L^{1}(\lambda)\left([\mathbb{N}\right.$, $\mathbb{N 1})$. Therefore $B_{L^{\infty}(\lambda)}$ separates these two classes. We use the a-thin sets in the following manner: Start with a set $W$ which for a sequence $\mathbf{a}=\left(a_{n}\right)_{n}$ is an a-thin set. Denote also by \|\|$_{n}$ the equivalent norm defined by the set $2^{n} W+a_{n} B_{X}$ and suppose that there exists a $d$-product norm on $\Omega_{00}=\left(\prod_{n=1}^{\infty}\left(X,\|\|_{n}\right)\right)_{00}$ which is block H.I. (Definition 3.3). Then the diagonal space $\Delta \tilde{X}$ is a H.I. space. Therefore the use of thin or a-thin sets is the essential tool to construct H.I. interpolation spaces.

The next three sections are devoted to the construction of a-thin (or thin) norming sets. We recall that a bounded set $W$ in a Banach space norms a subspace $Y$ of $X^{*}$ if there exists a constant $C>0$ such that for every $x^{*}$ in $Y$, $\left\|x^{*}\right\| \leq C \sup \left\{\left|x^{*}(w)\right|: w \in W\right\}$. Our goal is, given a member $A$ of a certain class of separable Banach spaces, to construct a corresponding space $X_{A}$ and $W$ an a-thin subset of $X_{A}$ which norms a subspace $Y$ of $X_{A}^{*} w^{*}$-isometric to $A^{*}$. It does not seem obvious how to construct such a $W$ even for concrete Banach spaces. However there were indications in the literature for the existence of such sets. We mention a result due to J. Bourgain in Radon-Nikodym theory that describes a similar phenomenon. It is proven in $[\mathrm{Bo} 2$ that if $K$ is a bounded convex set in a Banach space and $\varepsilon>0$ such that the convex combinations of slices of $K$ have diameter greater than $\varepsilon$, then the set $K$ norms a subspace of $X^{*}$ isomorphic to $\ell^{1}(\mathbb{N})$. It was somehow expected that one can construct such sets $K$, with the additional property that they be thin sets. Our construction concerns spaces $A$ with an unconditional basis. For such a space $A$ we consider an auxiliary space $X_{A}$ which is of the form $\left(\sum \oplus \ell^{1}\left(k_{n}\right)\right)_{A}$ and we "spread" the positive part of the unit ball of $A$ on the branches of an appropriate tree in the ball of $X_{A}$. Thus after defining such a set $K$, the set $W$ is the set $\overline{c o}(K \cup-K)$. As a result of this construction it easily follows that $W$ norms a subspace of $X_{A}^{*}$ isometric to $A^{*}$. The difficult part is to show that $W$ is an a-thin set. This is not always true. For example if $A$ contains $\ell^{1}(\mathbb{N})$, then $W$ is not an a-thin set. 
In the fourth section of the paper we prove that $W$ is an a-thin set for some special classes of reflexive Banach spaces with an unconditional basis, which include the classical spaces $L^{p}(\lambda), 1<p<\infty$.

In the fifth section we show that every reflexive space $A$ with an unconditional basis contains a block subspace $B$ such that the set $W$ in $X_{B}$ is an a-)thin set. This is the most general result that follows from our constructions. The proof depends on some earlier results from [AMT] on the summability of weakly null sequences.

To explain the role of a-thin (or thin) norming sets, let us assume that for a sequence $\left(X_{n}\right)_{n \in \mathbb{N}}$ of separable Banach spaces there exists a $d$-product norm on $\Omega_{00}=\left(\prod_{n=1}^{\infty} X_{n}\right)_{00}$ which is block-H.I. In that case, as we mentioned earlier, the diagonal space $\Delta \tilde{X}_{A}$ defined by the a-thin set $W$ in $X_{A}$ and the block-H.I. $d$-product norm is a H.I. space. Further on, since $W$ norms a subspace of $X_{A}^{*} w^{*}$-isometric to $A^{*}$ we get that $A^{*}$ is $w^{*}$-isometric to a subspace of $\left(\Delta \tilde{X}_{A}\right)^{*}$. This implies that $A$ is a quotient of $\Delta \tilde{X}_{A}$.

The sixth section contains the proof of the fact that the set $W$, defined for the space $c_{0}(\mathbb{N})$, is a thin set.

The seventh section is devoted to the general construction of block-H.I. $d$-product norms. In particular we prove that for every sequence $\left(X_{n}\right)_{n \in \mathbb{N}}$ of separable Banach spaces there exists a $d$-product norm on the space $\Omega_{00}=\left(\prod_{n=1}^{\infty} X_{n}\right)_{00}$ which is block-H.I. This means that for every block normalized sequence $\left(\tilde{x}_{n}\right)_{n \in \mathbb{N}}$ in $\Omega_{00}$ the Banach space $\overline{\operatorname{span}}\left[\left(\tilde{x}_{n}\right)_{n \in \mathbb{N}}\right]$ is a H.I. space. The construction of such a norm is very similar to the corresponding constructions in the already existing examples. For somebody experienced in such constructions it will be clear that it is possible to use either the Gowers-Maurey scheme ([GM]) or the asymptotic $\ell^{1}$ scheme ([AD2] ) and obtain variations of block-H.I. $d$-product norms. We follow the scheme presented in the construction of H.I. spaces by Gowers and Maurey with few modifications in the definition of the norm.

In the eight section the final results, including the results mentioned above, are presented.

\section{Direct PRoducts-Diagonal SPACES}

In this section we present a generalization of Davis-Figiel-Johnson-Pelczynski's interpolation method, by extending this to cases where the connecting external norm is not necessarily unconditional. We will use this in the next sections to construct interpolation spaces which are Hereditarily Indecomposable.

Notation. Let $\left(X_{n},\|\|_{n}\right)_{n \in \mathbb{N}}$ be a sequence of Banach spaces, and let $\Omega=\prod_{n=1}^{\infty} X_{n}$ be their Cartesian product. The support of a vector $\tilde{x} \in \Omega$, denoted by $\operatorname{supp}(\tilde{x})$, is the set of all $n \in \mathbb{N}$ such that $\tilde{x}(n) \neq 0$. We set $\Omega_{00}=\{\tilde{x} \in \Omega:|\operatorname{supp}(\tilde{x})|<\infty\}$. The range of a vector $\tilde{x} \in \Omega$, denoted by range $(\tilde{x})$, is the interval of integers [minsupp $\tilde{x}$, maxsupp $\tilde{x}]$. For any $A \subseteq \mathbb{N}$ we define a linear transformation $P_{A}: \Omega \rightarrow \Omega$ by $P_{A}(\tilde{x})(n)=\left\{\begin{array}{ll}\tilde{x}(n) & \text { if } n \in A \\ 0 & \text { if } n \notin A\end{array}\right.$. We also set $P_{n}=P_{\{1, \ldots, n\}}, \quad P_{n^{c}}=P_{\{n+1, \ldots\}}$, 
$\pi_{n}=P_{\{n\}}, E \tilde{x}=P_{E}(\tilde{x})$. For any $k \in \mathbb{N}$ we denote by $i_{k}: X_{k} \rightarrow \Omega_{00}$ the natural embedding defined by $i_{k}(x)(n)=\left\{\begin{array}{ll}x & \text { if } n=k \\ 0 & \text { if } n \neq k\end{array}\right.$.

Definition 2.1. A Banach space $(\tilde{X},\|\|)$ is called a d-product of the sequence $\left(X_{n},\|\|_{n}\right)_{n \in \mathbb{N}}$ if the following conditions are satisfied.

1. $\Omega_{00} \subseteq \tilde{X} \subseteq \Omega$ and $\Omega_{00}$ is a dense subset of $\tilde{X}$.

2. $\|x\|_{n}=\left\|i_{n}(x)\right\|$, for every $n \in \mathbb{N}$ and $x \in X_{n}$.

3. For any $n \in \mathbb{N}$ and $\tilde{x} \in \tilde{X}, P_{n}: \tilde{X} \rightarrow \tilde{X}$ is a bounded transformation and $\tilde{x}=\lim _{n \rightarrow \infty} P_{n} \tilde{x}$.

If $\left(X_{n},\|\|_{n}\right)_{n \in \mathbb{N}}$ is a sequence of Banach spaces and $A$ is a Banach space with 1-unconditional basis $\left(e_{n}\right)_{n \in \mathbb{N}}$, then

$$
\left(\prod_{n=1}^{\infty} X_{n}\right)_{A}=\left\{\left(x_{n}\right)_{n \in \mathbb{N}} \in \prod_{n=1}^{\infty} X_{n}:\left\|\sum_{n=1}^{\infty}\right\| x_{n}\left\|_{n} e_{n}\right\|_{A}<\infty\right\}
$$

with norm $\left\|\left(x_{n}\right)_{n \in \mathbb{N}}\right\|=\left\|\sum_{n=1}^{\infty}\right\| x_{n}\left\|_{n} e_{n}\right\|_{A}$ is an example of a $d$-product of the sequence $\left(X_{n}\right)_{n \in \mathbb{N}}$.

If $\tilde{X}$ is a $d$-product of $\left(X_{n},\|\|_{n}\right)_{n \in \mathbb{N}}$, then by the uniform boundedness principle there exists $C>0$ such that $\left\|P_{n} \tilde{x}\right\| \leq C\|\tilde{x}\|$, for any $n \in \mathbb{N}$ and $\tilde{x} \in \tilde{X}$. Therefore the sequence $\left(X_{n}\right)_{n \in \mathbb{N}}$ is a Schauder decomposition of the space $\tilde{X}$ (for a detailed study of this notion see [LT], pp. 47-52).

The family $\left(P_{n}\right)_{n=1}^{\infty}$ satisfies the following properties:

(P1) $P_{n} \circ P_{m}=P_{\min \{m, n\}},\left(\right.$ P2) $\sup _{n \in \mathbf{N}}\left\|P_{n}\right\|<\infty,(\mathbf{P 3})$ For every $x \in X, x=$ $\lim _{n \rightarrow \infty} P_{n} x$, or equivalently, $(\mathbf{P 3})^{\prime} \quad X=\overline{n \in \mathbf{N}}$

Conversely, if a Banach space $X$ admits a family $\left(P_{n}: X \rightarrow X\right)_{n=1}^{\infty}$ of linear operators satisfying $(\mathbf{P 1}),(\mathbf{P 2}),(\mathbf{P 3})$, then the space $X$ is the $d$-product of the family $\left(\left(P_{n}-P_{n-1}\right)(X)\right)_{n=1}^{\infty}$ where $P_{0}=0$. The norm \|\| of the space $\tilde{X}$ is called:

(a) C-bimonotone if there is a $C>0$ such that $\left\|P_{A}(\tilde{x})\right\| \leq C\|\tilde{x}\|$ for any interval $A \subseteq \mathbb{N}$ and any $\tilde{x} \in \tilde{X}$. It follows from the definition that every $d$-product norm is $C$-bimonotone for some $C$;

(b) unconditional if there is a $C>0$ such that $\left\|P_{A}(\tilde{x})\right\| \leq C\|\tilde{x}\|$ for any $A \subseteq \mathbb{N}$ and any $\tilde{x} \in \tilde{X}$;

(c) boundedly complete if for any $\tilde{x} \in \Omega, \sup _{n \in \mathbb{N}}\left\|P_{n} \tilde{x}\right\|<\infty$ implies that $\tilde{x} \in \tilde{X}$;

(d) shrinking if for any $\tilde{x}^{*} \in \tilde{X}^{*}, \tilde{x}^{*}=\lim _{n \rightarrow \infty} P_{n}^{*} \tilde{x}^{*}$, where $P_{n}^{*} \tilde{x}^{*}(x)=\sum_{i=1}^{n} \tilde{x}^{*}\left(\pi_{i}(\tilde{x})\right)$ $=\tilde{x}^{*}\left(P_{n} \tilde{x}\right)$.

Since $\left(P_{n}^{*}\right)_{n=1}^{\infty}$ satisfies $(\mathbf{P 1})$ and $(\mathbf{P} 2)$ the norm of $\tilde{X}$ is shrinking if and only if $\tilde{X}^{*}$ is a $d$-product of the sequence $\left(X_{n}^{*}\right)_{n=1}^{\infty}$. In the sequel we shall assume that \|\| is always 1-bimonotone and therefore $\|\tilde{x}\|_{\infty} \leq\|\tilde{x}\| \leq\|\tilde{x}\|_{1}$, where $\|\tilde{x}\|_{\infty}=$ $\sup _{n \in \mathbb{N}}\|\tilde{x}(n)\|_{n},\|\tilde{x}\|_{1}=\sum_{n=1}^{\infty}\|\tilde{x}(n)\|_{n}$. We notice that the space $\tilde{X}$ is reflexive if and only if each $X_{n}$ is reflexive and the norm of $\tilde{X}$ is boundedly complete and shrinking. (The proof is similar to that of the corresponding James' theorem for spaces with Schauder basis [LT.) 
Definition 2.2. Let $\left(X_{n},\|\|_{n}\right)_{n \in \mathbb{N}}$ be a sequence of Banach spaces such that $X_{n}=$ $X_{1}$ and \|\|$_{n}$ is equivalent to \|\|$_{1}$ for any $n \in \mathbb{N}$. Let $\tilde{X}$ be any $d$-product of the sequence $\left(X_{n},\|\|_{n}\right)_{n \in \mathbb{N}}$. The diagonal space $\Delta \tilde{X}$ of $\tilde{X}$ is the (closed) subspace of $\tilde{X}$ consisting of all $\tilde{x} \in \tilde{X}$ such that $\tilde{x}(n)=\tilde{x}(1)$, for any $n \in \mathbb{N}$.

Define $J: \Delta \tilde{X} \rightarrow X_{1}, I: \Delta \tilde{X} \rightarrow \tilde{X}, \pi_{1}: \tilde{X} \rightarrow X_{1}$ by $J(\tilde{x})=\tilde{x}(1), I(\tilde{x})=$ $\tilde{x}, \pi_{1}(\tilde{x})=\tilde{x}(1)$. Then $J=\pi_{1} \circ I$ and, moreover, it is a $1-1$, linear, continuous transformation.

If the norm of $\tilde{X}$ is boundedly complete and shrinking, then $J$ has the interesting property that $J^{* *}$ is $1-1$ and it is a Tauberian operator. The latter means that $\left(J^{* *}\right)^{-1}\left(X_{1}\right) \subseteq \Delta \tilde{X}$. We can easily prove that if $T: X \rightarrow Y$ is a Tauberian operator, then $T(W)$ is a relatively weakly compact subset of $Y$ iff $W$ is a relatively weakly compact subset of $X([\mathbf{N}])$. The notion of a Tauberian operator is introduced in [KW] and for a study and characterizations of Tauberian operators see [N], [N1, [NR.

Notation. a) For $A, B$ finite non-empty subsets of $\mathbb{N}$ we write $A<B$ iff $\max A<$ $\min B$.

b) If $\tilde{x}, \tilde{y} \in\left(\prod_{n=1}^{\infty} X_{n}\right)_{00}$, we write $\tilde{x}<\tilde{y}$ iff $\operatorname{supp}(\tilde{x})<\operatorname{supp}(\tilde{y})$.

c) A sequence $\left(\tilde{x}_{n}\right)_{n \in \mathbb{N}}$ of non-zero vectors of $\left(\prod_{n=1}^{\infty} X_{n}\right)_{00}$ is said to be a block sequence if $\tilde{x}_{n}<\tilde{x}_{n+1}$ for any $n \in \mathbb{N}$.

d) If $\tilde{X}$ is any $d$-product of a sequence $\left(X_{n},\|\|_{n}\right)_{n \in \mathbb{N}}$ of Banach spaces, then a block subspace of $\tilde{X}$ is the closed linear span of a block sequence $\left(\tilde{x}_{k}\right)_{k \in \mathbb{N}}$.

Definition 2.3. a) A Banach space $X$ is called a hereditarily indecomposable space or H.I. space if there is no subspace of $X$ that can be decomposed as a linear topological direct sum of two infinite-dimensional subspaces, or equivalently for any pair of infinite-dimensional subspaces $Y, Z$ of $X$ and any $\varepsilon>0$ there exist $y \in Y, z \in Z$ such that $\|y-z\|<\varepsilon\|y+z\|$.

b) A $d$-product $\tilde{X}$ is called a block-H.I. space if any block subspace of $\tilde{X}$ is an H.I. space.

Proposition 2.1. Let $\tilde{X}$ be any d-product of a sequence $\left(X_{n},\|\|_{n}\right)_{n \in \mathbb{N}}$ of Banach spaces such that $X_{n}=X_{1}$ and \|\|$_{n}$ is equivalent to \|\|$_{1}$ for any $n \in \mathbb{N}$. If $\tilde{X}$ is a block-H.I. space and the operator $J: \Delta \tilde{X} \rightarrow X_{1}$ is strictly singular, then $\Delta \tilde{X}$ is a H.I. space.

Proof. We recall that the space $\tilde{X}$ is endowed with 1-bimonotone norm and let us suppose that $\Delta \tilde{X}$ is not a H.I. space. Then there exist $Y_{1}, Y_{2}$ infinite-dimensional closed subspaces of $\Delta \tilde{X}$ such that $Y_{1} \cap Y_{2}=\{0\}$ and $Y_{1}+Y_{2}$ is also closed. This is equivalent to the existence of some $\delta>0$ such that for every $\tilde{y}_{1} \in Y_{1}, \tilde{y}_{2} \in Y_{2}$, $\frac{1}{2}<\left\|\tilde{y}_{i}\right\| \leq \frac{3}{2}, i=1,2$, we have that $\left\|\tilde{y}_{1}-\tilde{y}_{2}\right\|>\delta$. We may also assume that $\delta<\frac{1}{4}$.

Claim. There exists a block sequence $\left(\tilde{z}_{i}\right)_{i=1}^{\infty}$ in $\tilde{X}$ such that setting

$$
Z_{1}=\overline{\operatorname{span}}\left[\left(\tilde{z}_{2 j}\right)_{j=1}^{\infty}\right], \quad Z_{2}=\overline{\operatorname{span}}\left[\left(\tilde{z}_{2 j-1}\right)_{j=1}^{\infty}\right]
$$

then for every $\tilde{w}_{1} \in S_{Z_{1}}, \tilde{w}_{2} \in S_{Z_{2}}$ there are $\tilde{u}_{1} \in Y_{1}, \tilde{u}_{2} \in Y_{2}$ satisfying $\left\|\tilde{w}_{i}-\tilde{u}_{i}\right\|<$ $\frac{\delta}{4}$ for $i=1,2$. Hence we get $\left\|\tilde{w}_{1}-\tilde{w}_{2}\right\|>\frac{\delta}{4}$. 
If such a sequence $\left(\tilde{z}_{i}\right)_{i=1}^{\infty}$ existed, then for the block subspace $Z=\overline{\operatorname{span}}\left[\left(\tilde{z}_{i}\right)_{i=1}^{\infty}\right]$ we would have that $Z=Z_{1} \oplus Z_{2}$, contradicting the assumption that $\tilde{X}$ is a block H.I. space.

To prove the claim we begin with the following two observations:

(1) Since \|\|$_{1},\|\|_{n}$ are equivalent, for every $\varepsilon>0$ and $n \in \mathbb{N}$ there exists $\varepsilon^{\prime}>0$ such that for every $\tilde{y}=(y, y, \ldots)$ in $\Delta \tilde{X}$ if $\|y\|_{1}<\varepsilon^{\prime}$, then $\sum_{k=1}^{n}\|y\|_{k}<\varepsilon$ and hence $\left\|P_{n}(\tilde{y})\right\|<\varepsilon$.

(2) Since $J$ is a strictly singular operator it follows that for every $\tilde{Y}$ infinitedimensional subspace of $\Delta \tilde{X}$ and every $\varepsilon^{\prime}>0$ there exists $\tilde{y} \in \tilde{Y},\|\tilde{y}\|=1$ and $\|J(\tilde{y})\|_{1}<\varepsilon^{\prime}$.

These two observations permit us to apply the standard sliding hump argument and obtain sequences $\left(\tilde{y}_{i}\right)_{i=1}^{\infty},\left(\tilde{z}_{i}\right)_{i=1}^{\infty}$ so that the following properties are satisfied:

- $\tilde{y}_{2 j-1} \in Y_{1}, \tilde{y}_{2 j} \in Y_{2}$ for $j=1,2, \ldots$ and $\left\|\tilde{y}_{i}\right\|=1$, for all $i \in \mathbb{N}$.

- $\left(\tilde{z}_{i}\right)_{i=1}^{\infty}$ is a block sequence of $\tilde{X}$ and $\left\|\tilde{y}_{i}-\tilde{z}_{i}\right\|<\frac{\delta}{2^{2+4}}$, for all $i \in \mathbb{N}$.

We set $Z_{1}=\overline{\operatorname{span}}\left[\left(\tilde{z}_{2 j-1}\right)_{j=1}^{\infty}\right], Z_{2}=\overline{\operatorname{span}}\left[\left(\tilde{z}_{2 j}\right)_{j=1}^{\infty}\right]$. Let $\tilde{w}_{1} \in Z_{1},\left\|\tilde{w}_{1}\right\|=1$. Then $\tilde{w}_{1}=\sum_{j=1}^{\infty} \alpha_{j} \tilde{z}_{2 j-1}$ and we set $\tilde{u}_{1}=\sum_{j=1}^{\infty} \alpha_{j} \tilde{y}_{2 j-1}$. We observe that $\left\|\tilde{u}_{1}-\tilde{w}_{1}\right\| \leq$ $\sum_{j=1}^{\infty}\left|\alpha_{j}\right| \frac{\delta}{2^{2 j+3}}<\frac{\delta}{4}$. The last inequality holds from the monotonicity of the norm of $\tilde{X}$. Therefore $\tilde{u}_{1} \in Y_{1}$ and $1-\frac{\delta}{4}<\left\|\tilde{u}_{1}\right\|<1+\frac{\delta}{4}$. For the same reason for $\tilde{w}_{2} \in S_{Z_{2}}$ there exists $\tilde{u}_{2} \in Y_{2}$ with $1-\frac{\delta}{4}<\left\|\tilde{u}_{2}\right\|<1+\frac{\delta}{4}$ and $\left\|\tilde{u}_{2}-\tilde{w}_{2}\right\|<\frac{\delta}{4}$. This proves the claim and as we have already mentioned it leads to a contradiction.

Definition 2.4. Let $W$ be a bounded convex symmetric non-empty subset of a Banach space $(X,||||||)$, and $\mathbf{a}=\left(a_{n}\right)_{n=1}^{\infty}$ a null sequence of positive numbers. We set $W_{n}=2^{n} W+a_{n} B_{X}$ and denote by \|\|$_{n}$ the gauge of $W_{n}$. Then for every $d$-product norm of the sequence $\left(X,\|\|_{n}\right)_{n \in \mathbb{N}}$ we shall call the diagonal space $\Delta \tilde{X}$ an $(\mathbf{a}, W, \tilde{X})$-diagonal space or simply an $(\mathbf{a}, W, \tilde{X})$-space.

In case $a_{n}=2^{-n}$ for every $n$ and $\tilde{X}=\left(\sum_{n=1}^{\infty} \oplus X_{n}\right)_{\ell^{2}}$, then the $(\mathbf{a}, W, \tilde{X})$ diagonal space is the interpolation space introduced in [DFJP].

The following proposition can be readily proven.

Proposition 2.2. Let $\Delta \tilde{X}$ be an $(\mathbf{a}, W, \tilde{X})$-diagonal space of the sequence $\left(X,\|\|_{n}\right)_{n \in \mathbb{N}}$. Then the following hold:

(a) $W \subseteq J B_{\Delta \tilde{X}}$.

(b) For every $\varepsilon>0$ there exists $\lambda>0$ such that $J B_{\Delta \tilde{X}} \subseteq \lambda W+\varepsilon B_{X}$.

Definition 2.5. Let $(X,\|\|)$ be a Banach space, $A, B \subseteq X$ and $\varepsilon>0$. We say that

(i) the set $A \varepsilon$-absorbs $B$ if there exists a $\lambda>0$ such that $B \subseteq \lambda A+\varepsilon B_{X}$;

(ii) the set $A$ almost absorbs $B$ if $A \varepsilon$-absorbs $B$ for any $\varepsilon>0$.

The following lemma is due to A. Grothendieck $(\overline{\mathrm{Gr}}])$ and for a proof we refer to [D]. We are going to use this lemma in the proof of the last proposition of this section. Although we will not use these results we present them to illustrate that the factorization results in DFJP can be extended in the case of not necessarily unconditional $d$-products. 
Lemma 2.3. Let $K$ be a weakly closed subset of the Banach space $X$. Suppose that for each $\varepsilon>0$ there exists a weakly compact set $K_{\varepsilon}$ in $X$ such that $K \subset K_{\varepsilon}+\varepsilon B_{X}$. Then $K$ is weakly compact.

Proposition 2.4. Let $\Delta \tilde{X}$ be an $(\mathbf{a}, W, \tilde{X})$-diagonal space such that the norm of $\tilde{X}$ is boundedly complete and shrinking. Then $\Delta \tilde{X}$ is reflexive if and only if $W$ is a relatively weakly compact subset of $X$.

Proof. If $W$ is a relatively weakly compact subset of $X$, then by Proposition 2.2 (b) $W$ almost absorbs the set $J B_{\Delta \tilde{X}}$ and hence from Lemma 2.3 $J B_{\Delta \tilde{X}}$ is a relatively weakly compact set. Since $J$ is a Tauberian operator, we get that $B_{\Delta \tilde{X}}$ is a relatively weakly compact subset of $\Delta \tilde{X}$.

\section{THIN SETS}

In this section we initiate the study of thin and a-thin sets that we will continue in the next two sections. The notion of a thin set was introduced in [N], [N1], and that of an a-thin set, which is weaker, was suggested to us by B. Maurey who also showed us how to prove that the unit ball of $L^{\infty}(\lambda)$ is an a-thin subset of $L^{1}(\lambda)$. It was observed by Rosenthal ([N], [N1]) that this set is not a thin subset of $L^{1}(\lambda)$.

Thin or a-thin sets are important for our considerations since the interpolation diagonal space defined by an a-thin set (and hence by a thin set) and an appropriate external norm is, as we will show in Proposition 3.1, an H.I. space.

Definition 3.1. a) A bounded convex symmetric non-empty subset $W$ of a Banach space $X$ is said to be a thin set if $W$ does not almost-absorb the unit ball $B_{Y}$ of any infinite-dimensional closed subspace $Y$ of $X$ (i.e. for any $Y$, infinite-dimensional closed subspace of $X$, there exists $\varepsilon>0$ such that for all $\left.\lambda>0, B_{Y} \nsubseteq \lambda W+\varepsilon B_{X}\right)$.

b) An operator $T: Z \rightarrow X$ is called thin if $T B_{Z}$ is a thin subset of $X$.

Remark 3.1. (a) There are several examples of thin sets. The closed absolutely convex hull of the basis $\left(e_{n}\right)_{n=1}^{\infty}$ in any $\ell^{p}(\mathbb{N})$, for $1<p<\infty$, or in $c_{0}(\mathbb{N})$ is a thin set.

(b) Every thin operator is always strictly singular. The converse is not true. Indeed, as shown in $\left[\mathbb{N}\right.$ and mentioned above, the set $B_{L^{\infty}(\lambda)}$ in $L^{1}(\lambda)$ is not a thin set while the identity operator $I: L^{\infty}(\lambda) \rightarrow L^{1}(\lambda)$ is a strictly singular operator.

(c) It follows easily from the definition that if $W$ is a thin subset of a Banach space $X$ and $\mathbf{a}=\left(a_{n}\right)_{n \in \mathbb{N}}$ is a null sequence of positive numbers, then the sequence of equivalent norms $\left(\|\|_{n}\right)_{n \in \mathbb{N}}$ on $X$ defined by the Minkowski gauges $\left(2^{n} W+a_{n} B_{X}\right)_{n \in \mathbb{N}}$ is not uniformly bounded on $B_{Z}$, for every infinite-dimensional closed subspace $Z$ of $X$.

Definition 3.2. a) Let $\mathbf{a}=\left(a_{n}\right)_{n=1}^{\infty}$ be a null sequence of positive numbers. The set $W$ is said to be an a-thin subset of $X$ if for any $Y$, infinite-dimensional closed subspace of $X, \sup _{n \in \mathbb{N}}\left\{\sup _{y \in B_{Y}}\|y\|_{n}\right\}=\infty$, where \|\|$_{n}$ is the gauge of $W_{n}=$ $2^{n} W+a_{n} B_{X}$. Equivalently, a set $W$ is an a-thin subset of $X$ if for any $Y$, infinitedimensional closed subspace of $X$, and every $\lambda>0$ there exists an $n \in \mathbb{N}$ such that $B_{Y} \not \lambda\left(2^{n} W+a_{n} B_{X}\right)$.

b) An operator $T: Z \rightarrow X$ is called a-thin if $T B_{Z}$ is an a-thin subset of $X$.

Remark 3.2. Every thin subset of $X$ is a-thin for every null positive sequence a and every thin operator is a-thin for every null positive sequence a. An a-thin operator is strictly singular. In particular if $W$ is an a-thin set, then the operator 
$J: \Delta \tilde{X} \rightarrow X$ is strictly singular. Combining these observations with Proposition 2.1 we get

Proposition 3.1. Let $\Delta \tilde{X}$ be the $(\mathbf{a}, W, \tilde{X})$-diagonal space defined by an $\mathbf{a}$-thin set $W$ of a Banach space $X$ and such that $\tilde{X}$ is a block-H.I. space. Then $\Delta \tilde{X}$ is a H.I. space.

The next two results, due to B. Maurey, will enable us to show that $B_{L^{\infty}(\mu)}$ is an a-thin subset of $L^{1}(\mu)$ for every probability measure $\mu$.

Lemma 3.2. Let $(\Omega, \mu)$ be a probability space. Let $Z$ be an infinite-dimensional subspace of $L^{2}(\mu)$ such that $Z \subset L^{p}(\mu)$ for every $p<\infty$ and let $C_{p}(Z)=$ $\sup \left\{\|f\|_{p}: f \in Z\right.$ and $\left.\|f\|_{2} \leq 1\right\}$. Then $\liminf _{p \rightarrow+\infty} \frac{C_{p}(Z)}{\sqrt{p}} \geq e^{-\frac{1}{2}}$.

Proof. Let us choose an orthonormal basis $\mathbf{f}=\left(f_{1}, \ldots, f_{n}, \ldots\right)$ for our subspace $Z$ of $L^{2}(\mu)$ and let us fix $p>2$. By assumption,we have $Z \subset \overline{\operatorname{span}}\left[f_{j}\right]_{j=1}^{\infty} \subset L^{p}(\mu)$ and $C_{p}(Z)$ is equal to the smallest constant $K$ such that

$$
\left\|\sum_{j=1}^{n} c_{j} f_{j}\right\|_{p} \leq K\left\|\sum_{j=1}^{n} c_{j} f_{j}\right\|_{2}=K\left(\sum_{j=1}^{n}\left|c_{j}\right|^{2}\right)^{\frac{1}{2}}
$$

for all $n \geq 1$ and for all real scalars $\left(c_{j}\right)$ (if no such constant exists, we let $C_{p}(Z)=$ $\infty)$.

Let $\mathbf{g}=\left(g_{1}, \ldots, g_{n}, \ldots\right)$ be a sequence of independent Gaussian random variables on $(\Omega, \mu)$ with common distribution $(2 \pi)^{-\frac{1}{2}} e^{-\frac{x^{2}}{2}} d x$ and let $G \subset L^{2}(\mu)$ be the closed subspace spanned by $\mathbf{g}$. We know that all linear combinations $\sum_{j} c_{j} g_{j}$ such that $\sum_{j}\left|c_{j}\right|^{2}=1$ have the same distribution, namely that of $g_{1}$; therefore

$$
\left\|\sum_{j=1}^{n} c_{j} g_{j}\right\|_{p}=C_{p}(G)\left(\sum_{j=1}^{n}\left|c_{j}\right|^{2}\right)^{\frac{1}{2}}
$$

for all real scalars and

$$
C_{p}(G)=\left\|g_{1}\right\|_{p}=\left(\int_{-\infty}^{+\infty}|x|^{p} e^{-\frac{x^{2}}{2}} \frac{d x}{\sqrt{2 \pi}}\right)^{\frac{1}{p}}=\left(\frac{2^{\frac{p}{2}}}{\sqrt{\pi}} \Gamma\left(\frac{p+1}{2}\right)\right)^{\frac{1}{p}} \sim \sqrt{\frac{p}{e}}
$$

as $p \rightarrow \infty$, using Stirling's formula.

Assume that $C_{p}(Z)<\infty$. Let us consider $\sum_{j=1}^{n} f_{j}(s) g_{j}(t)$. Then for $p>2$ by (1) we get that

$$
\begin{aligned}
\iint\left|\sum_{j=1}^{n} f_{j}(s) g_{j}(t)\right|^{p} d s d t & =\left(C_{p}(G)\right)^{p} \int\left(\sum_{j=1}^{n}\left|f_{j}(s)\right|^{2}\right)^{\frac{p}{2}} d s \\
& \geq\left(C_{p}(G)\right)^{p}\left(\int\left(\sum_{j=1}^{n}\left|f_{j}(s)\right|^{2}\right) d s\right)^{\frac{p}{2}}=n^{\frac{p}{2}}\left(C_{p}(G)\right)^{p} .
\end{aligned}
$$


On the other hand,

$$
\iint\left|\sum_{j=1}^{n} f_{j}(s) g_{j}(t)\right|^{p} d s d t \leq\left(C_{p}(Z)\right)^{p} \int\left(\sum_{j=1}^{n}\left|g_{j}(t)\right|^{2}\right)^{\frac{p}{2}} d t
$$

and

$$
\varphi(p, n)=\int\left(\sum_{j=1}^{n}\left|g_{j}(t)\right|^{2}\right)^{\frac{p}{2}} d t=\int_{\mathbb{R}^{n}}\|x\|^{p} d \gamma_{n}(x)=v_{n-1} \int_{0}^{+\infty} r^{p} e^{-\frac{r^{2}}{2}} r^{n-1} \frac{d r}{(2 \pi)^{\frac{n}{2}}}
$$

where $v_{n-1}$ is the surface area of the unit sphere in $\mathbb{R}^{n}$. By change of variable,

$$
\varphi(p, n)=v_{n-1} \frac{2^{\frac{p}{2}+\frac{n}{2}-1}}{(2 \pi)^{\frac{n}{2}}} \int_{0}^{+\infty} u^{\frac{p}{2}+\frac{n}{2}-1} e^{-u} d u=v_{n-1} \frac{2^{\frac{p}{2}-1}}{\pi^{\frac{n}{2}}} \Gamma\left(\frac{n+p}{2}\right) .
$$

Taking $p=2$ gives $n=\varphi(2, n)=\frac{v_{n-1}}{\pi^{\frac{n}{2}}} \Gamma\left(\frac{n}{2}+1\right), \frac{\varphi(p, n)}{n}=\frac{\varphi(p, n)}{\varphi(2, n)}=2^{\frac{p}{2}-1} \frac{\Gamma\left(\frac{n}{2}+\frac{p}{2}\right)}{\Gamma\left(\frac{n}{2}+1\right)}$ so that $\varphi(p, n)=2^{\frac{p}{2}} \frac{\Gamma\left(\frac{n}{2}+\frac{p}{2}\right)}{\Gamma\left(\frac{n}{2}\right)}$.

Summing up our information, $n^{\frac{1}{2}} C_{p}(G) \leq C_{p}(Z)(\varphi(p, n))^{\frac{1}{p}}$ or $C_{p}(G) \leq$ $\sqrt{\frac{2}{n}}\left(\frac{\Gamma\left(\frac{n}{2}+\frac{p}{2}\right)}{\Gamma\left(\frac{n}{2}\right)}\right)^{\frac{1}{p}} C_{p}(Z)$

We know by Stirling's formula that for any fixed $x>0$ we have that $\Gamma(t+x) \sim$ $t^{x} \Gamma(t)$ when $t$ tends to $+\infty$. Applying this for $x=\frac{p}{2}$ we get $\lim _{n \rightarrow+\infty} \sqrt{\frac{2}{n}}\left(\frac{\Gamma\left(\frac{n}{2}+\frac{p}{2}\right)}{\Gamma\left(\frac{n}{2}\right)}\right)^{\frac{1}{p}}$ $=1$ and it follows that $C_{p}(G) \leq C_{p}(Z)$.

This says that the Gaussian case is the best possible inequality between the $L^{2}$ and $L^{p}$ norms. By $(2)$ we already know that $C_{p}(G)$ is equivalent to $\sqrt{\frac{p}{e}}$ and this concludes the proof.

Proposition 3.3. Let $(\Omega, \mu)$ be a probability space. The unit ball $B_{L^{\infty}(\mu)}$ is an a-thin subset of $L^{1}(\mu)$ for $\mathbf{a}=\left(a_{n}\right)_{n}$ with $a_{n}=\exp \left(-16^{n}\right)$.

Proof. Assume the contrary; then there exists an infinite-dimensional closed subspace $Z$ of $L^{1}(\mu)$ and $M>0$ such that $\frac{1}{M} B_{Z} \subset 2^{n} B_{L^{\infty}}+a_{n} B_{L^{1}}$, for every $n \in \mathbb{N}$. So, every $z \in Z$ with $\|z\|_{1} \leq \frac{1}{M}$ has a decomposition $z=z_{0}+z_{1}$ with $\left\|z_{0}\right\|_{\infty} \leq 2^{n}$ and $\left\|z_{1}\right\|_{1} \leq a_{n}$. This implies that $\mu\left(s \in \Omega:|z(s)|>2^{n+1}\right) \leq$ $\mu\left(s \in \Omega:\left|z_{1}(s)\right|>2^{n}\right) \leq 2^{-n} a_{n}$.

Thus for every $\lambda>2$ choosing $n \geq 0$ such that $2^{n+1}<\lambda \leq 2^{n+2}$ the last inequality implies that

$$
\mu(s \in \Omega:|z(s)|>\lambda) \leq 2^{-n} a_{n} \leq \frac{4}{\lambda} \exp \left(\frac{-\lambda^{4}}{4^{4}}\right) .
$$

Therefore, using the definition of the Gamma function we get

$$
\|z\|_{p}^{p} \leq 2^{p}+\int_{2}^{+\infty} p \lambda^{p-1} \frac{4}{\lambda} \exp \left(\frac{-\lambda^{4}}{4^{4}}\right) d \mu \leq 2^{p}+p 4^{p-1} \Gamma\left(\frac{p-1}{4}\right) .
$$


Applying Stirling's formula we have that $\|z\|_{p} \leq O\left(p^{\frac{1}{4}}\right)$, when $p \rightarrow+\infty$, uniformly for $z$ in the unit ball of $Z$. So $Z$ is a closed subspace of $L^{p}(\mu)$, for every $1 \leq p<\infty$, and $C_{p}(Z) \leq O\left(p^{\frac{1}{4}}\right)$, contradicting Lemma 3.2.

Definition 3.3. A bounded subset $W$ of a Banach space $X$ is called a C-norming set for a subspace $Z$ of $X^{*}, C>0$, if $\|z\| \leq C \sup _{w \in W}|z(w)|$, for any $z \in Z$. The set $W$ is called a norming set for the subspace $Z$ if it is $C$-norming for $Z$ for some $C>0$.

Proposition 3.4. (a) Let $\Delta \tilde{X}$ be an $(\mathbf{a}, W, \tilde{X})$-diagonal space, and let $W$ be a $C$-norming set for a subspace $Z$ of $X^{*}$. Then $Z$ is isomorphic to a closed subspace of $\Delta \tilde{X}^{*}$.

(b) Let $X, W$ be as before, and let $A$ be a Banach space such that $A^{*}$ is $w^{*}$ isomorphic to $Z$. Then $A$ is a quotient of $\Delta \tilde{X}$.

Proof. (a) Denote by $J^{*}$ the adjoint of the operator $J$. By our assumption for any $z \in Z$ there exists $w_{z} \in W$ such that $\|z\| \leq 2 C\left|z\left(w_{z}\right)\right|$. Since $W \subseteq B_{\Delta \tilde{X}}$, ||$J^{*} z|| \geq\left|J^{*} z\left(J^{-1}\left(w_{z}\right)\right)\right|=\left|z\left(w_{z}\right)\right| \geq \frac{1}{2 C}|| z||$. Therefore $\left.J^{*}\right|_{Z}$ is an isomorphism onto a subspace of $\Delta \tilde{X}^{*}$.

(b) Let $S: A^{*} \rightarrow Z$ be a $w^{*}$-continuous isomorphism. Then $J^{*} \circ S$ is also a $w^{*}$-continuous isomorphism; hence $\left(J^{*} \circ S\right)^{*}$ maps the space $\Delta \tilde{X}$ onto the space $A$.

\section{Thin norming Sets I (SPecial Reflexive Cases)}

This is the first section devoted to the a-thin (thin) norming sets. For a reflexive Banach space $A$ with an unconditional basis we shall define a space $X_{A}$ which is of the form $\left(\sum_{n=1}^{\infty} \oplus \ell^{1}\left(k_{n}\right)\right)_{A}$ and a symmetric convex closed subset $W$ of the unit ball of $X_{A}$ which norms a subspace of $X_{A}^{*}$ isometric to $A^{*}$, and for certain spaces $A$ we shall show that the set $W$ is an a-thin subset of $X_{A}$. The set $W$ is of the form $\overline{c o}(K \cup-K)$ where $K$ forms a tree in the branches of which we have "spread" in a regular way a dense subset of the positive part of the unit ball of $A$. The fact that such a $W$ norms $A^{*}$ is quite easy and it is true for all reflexive Banach spaces $A$ with an unconditional basis. To show that $W$ is an a-thin set is more difficult. For this we use two combinatorial results that we prove in this section. The first, Proposition 4.4, "the finite version", will be applied in the results of the next section, and the second, Proposition 4.6] "the infinite version", will be used in the present section. Property $(P)$ given in Definition 4.1, suggested by B. Maurey, is the main tool to show that for certain classes of reflexive spaces the corresponding set $W$ is an a-thin set. Thus for example we are able to prove that for spaces like $L^{p}(\lambda), \ell^{p}(\mathbb{N}), 1<p<\infty$, and others, the corresponding set $W$ is an a-thin set. Finally we prove a similar result for spaces with many complemented subspaces, suggested to us by N. Tomczak-Jaegermann.

The space $\mathbf{X}_{A}$. We begin with the definition of the space $X_{A}$ and the subspace $Y$ of $X_{A}^{*}$.

Consider a tree $\mathfrak{D}$ of height $\omega$ with a least element $\rho$ such that every $\delta$ in $\mathfrak{D}$ with height equal to $n$ has $3^{4(n+1)}+1$ immediate successors. We identify $\mathfrak{D}$ with the set of all finite sequences $\left(k_{1}, \ldots, k_{n}\right), n \in \mathbb{N}$, of natural numbers such that $0 \leq k_{i} \leq 3^{4 i}$ 
and with least element the empty sequence. Consider the usual partial order $\prec$ in $\mathfrak{D}$, i.e. $\left(k_{1}, \ldots, k_{n}\right) \prec\left(k_{1}^{\prime}, \ldots, k_{\ell}^{\prime}\right)$ iff $\left(k_{1}, \ldots, k_{n}\right)$ is an initial segment of $\left(k_{1}^{\prime}, \ldots, k_{\ell}^{\prime}\right)$.

A segment is a subset of $\mathfrak{D}$ of the form $d=\left[\delta_{1}, \delta_{2}\right]=\left\{\delta \in \mathfrak{D}: \delta_{1} \prec \delta \prec \delta_{2}\right\}$. A segment $d$ of the form $d=[\rho, \delta]$ is called an initial segment of $\mathfrak{D}$. For every segment $d=\left[\delta_{1}, \delta_{2}\right]$ we define $\operatorname{ext}(d)=\left[\rho, \delta_{2}\right]$, that is, the minimal initial segment containing the segment $d$.

A branch is a maximal linearly ordered subset of $\mathfrak{D}$. A branch is identified to an infinite sequence $\left(k_{i}\right)_{i \in \mathbb{N}}$ of natural numbers, where $0 \leq k_{i} \leq 3^{4 i}$.

The height of $\delta=\left(k_{1}, \ldots, k_{n}\right)$ is denoted $|\delta|$ and it is equal to $n$. We set $|\rho|=0$.

We denote by $c_{00}(\mathfrak{D})$ the linear space of all functions $f: \mathfrak{D} \rightarrow \mathbb{R}$ such that $\operatorname{supp}(f)=\{\delta \in \mathfrak{D}: f(\delta) \neq 0\}$ is a finite set. Also, denote by $e_{\delta}$ the characteristic function of $\{\delta\}, \delta \in \mathfrak{D}$. The vectors $\left(e_{\delta}\right)_{\delta \in \mathfrak{D}}$ form a Hamel basis for $c_{00}(\mathfrak{D})$. If $A, B$ are finite subsets of $\mathfrak{D}$ we write $A<B$ iff $\max \{|\alpha|: \alpha \in A\}<\min \{|\beta|: \beta \in B\}$, and $A \prec B$ iff $\alpha \prec \beta$ for any $\alpha \in A$ and any $\beta \in B$. If $f \in c_{00}(\mathfrak{D})$, we set range $(f)=\{\delta \in \mathfrak{D}: m \leq|\delta| \leq M\}$, where $m=\min \{|\delta|: \delta \in \operatorname{supp}(f)\}$ and $M=\max \{|\delta|: \delta \in \operatorname{supp}(f)\}$. If $f, g \in c_{00}(\mathfrak{D})$ we write $f<g$ iff $\operatorname{supp}(f)<\operatorname{supp}(g)$ and $f \prec g$ iff $\operatorname{supp}(f) \prec \operatorname{supp}(g)$.

For a given Banach space $A$ with an unconditional basis $\left(e_{n}\right)_{n \in \mathbb{N}}$ we define the following norm of $c_{00}(\mathfrak{D})$ :

For $f=\sum_{\delta \in \mathfrak{D}} \lambda_{\delta} e_{\delta} \in c_{00}(\mathfrak{D})$ we set $\|f\|=\left\|\sum_{n=0}^{\infty}\left(\sum_{|\delta|=n}\left|\lambda_{\delta}\right|\right) e_{n}\right\|_{A}$.

The space $X_{A}$ is the completion of $c_{00}(\mathfrak{D})$ with the above defined norm. It is clear that $X_{A}$ is isometric to $\left(\sum_{n=0}^{\infty} \oplus \ell^{1}\left(k_{n}\right)\right)_{A}$ for $k_{n}=\prod_{i=1}^{n}\left(3^{4 i}+1\right)$ and if $A$ has a shrinking basis, then $X_{A}^{*}=\left(\sum_{n=0}^{\infty} \oplus \ell^{\infty}\left(k_{n}\right)\right)_{A^{*}}^{A}$. If $A$ is a reflexive Banach space, then $X_{A}$ is also reflexive.

Set $y_{n}^{*}=\sum_{|\delta|=n} e_{\delta}^{*}$ and $Z=\overline{\operatorname{span}}\left[\left(y_{n}^{*}\right)_{n \in \mathbb{N}}\right]$. Then $\left\|y_{n}^{*}\right\|=1$ and it is easily verified that the map $T\left(y_{n}^{*}\right)=e_{n}^{*}$ extends to an isometry between the spaces $Z$ and $A^{*}$. Throughout this section we will denote by $A$ a reflexive space with a 1 -unconditional basis $\left(e_{n}\right)_{n \in \mathbb{N}}$.

The set W. For every $\delta=\left(k_{1}, \ldots, k_{n}\right)$ we set $a_{\delta}=\frac{k_{n}}{3^{4 n}}$.

For any infinite branch $\gamma$ of $\mathfrak{D}$ we denote by $x_{\gamma}$ the formal series $x_{\gamma}=\sum_{n \in \mathbb{N}} a_{\delta_{n}} e_{\delta_{n}}$, where $\delta_{n}=\left(k_{1}, \ldots, k_{n}\right) \in \mathfrak{D}$ is the initial segment of $\gamma$ with height equal to $n$. Also we set $\left\|\mid x_{\gamma}\right\|=\left\|\sum_{n=0}^{\infty} \frac{k_{n}}{3^{4 n}} e_{n}\right\|_{A}$. It follows from the definitions that if $\left\|\left|x_{\gamma} \|\right|<\infty\right.$, then $x_{\gamma} \in X_{A}$ and $\left\|x_{\gamma}\right\|=\|\| x_{\gamma} \| \mid$. We set $K=\left\{x_{\gamma}:\left\|x_{\gamma}\right\| \leq 1\right\}, W_{0}=\operatorname{co}(K \cup-K)$ and $W=\overline{W_{0}}$. The set $W$ is a closed bounded symmetric convex subset of $B_{X_{A}}$.

Lemma 4.1. The set $K$ (and hence the set $W$ ) is a $\frac{1}{4}$-norming set for the space $Z \subseteq X_{A}^{*}$.

Proof. Let $y^{*}=\sum_{k=1}^{\infty} \lambda_{k} y_{k}^{*} \in S_{Z}$; then $\left\|y^{*}\right\|=\left\|\sum_{k=1}^{\infty} \lambda_{k} e_{k}^{*}\right\|_{A^{*}}=1$. Define $z^{*}=$ $\sum_{k=1}^{\infty} \lambda_{k} e_{k}^{*} \in A^{*}$ and notice that $\left\|z^{*}\right\|_{A^{*}}=\left\|y^{*}\right\|=1$. Consider a vector $z=\sum_{k=1}^{\infty} \mu_{k} e_{k} \in$ 
$S_{A}$ such that $\left|z^{*}(z)\right|=1=\left|\sum_{k=1}^{\infty} \lambda_{k} \mu_{k}\right|$. Since $\left(e_{n}\right)_{n \in \mathbb{N}}$ is 1-unconditional and bimonotone, $1=\left|\sum_{k=1}^{\infty} \lambda_{k} \mu_{k}\right| \leq\left|\sum_{\mu_{k}>0} \lambda_{k} \mu_{k}\right|+\left|\sum_{\mu_{k}<0} \lambda_{k} \mu_{k}\right|$.

Assume that $\left|\sum_{\mu_{k}>0} \lambda_{k} \mu_{k}\right| \geq \frac{1}{2}$.

For any $k \in \mathbb{N}$ we define $n_{k}=0$ if $\mu_{k} \leq 0$, while if $\mu_{k}>0$, then $n_{k}$ is defined to be the unique natural number with the property $\frac{n_{k}}{3^{4 k}} \leq \mu_{k}<\frac{n_{k}+1}{3^{4 k}}$. Since $\left(e_{n}\right)_{n \in \mathbb{N}}$ is bimonotone, $\mu_{k} \leq 1$ for any $k \in \mathbb{N}$ and therefore $n_{k} \leq 3^{4 k}$ for any $k \in \mathbb{N}$.

Let $\gamma=\left(n_{1}, n_{2}, \ldots\right)$ be an infinite branch of $\mathfrak{D}$. Then $\left\|x_{\gamma}\right\|=\left\|\sum_{k=1}^{\infty} \frac{n_{k}}{34 k} e_{k}\right\|_{A} \leq$ $\left\|\sum_{k=1}^{\infty} \mu_{k} e_{k}\right\|_{A} \leq 1$, i.e. $\quad x_{\gamma} \in K$. Also, $\left|y^{*}\left(x_{\gamma}\right)\right|=\left|\sum_{k=1}^{\infty} \lambda_{k} \frac{n_{k}}{3^{4 k}}\right|=\left|\sum_{\mu_{k}>0} \lambda_{k} \frac{n_{k}}{3^{4 k}}\right| \geq$ $\left|\sum_{\mu_{k}>0} \lambda_{k} \mu_{k}\right|-\left|\sum_{\mu_{k}>0} \lambda_{k}\left(\mu_{k}-\frac{n_{k}}{3^{4 k}}\right)\right| \geq \frac{1}{2}-\sum_{k=1}^{\infty} \frac{1}{3^{4 k}}>\frac{1}{4}=\frac{1}{4}\left\|y^{*}\right\|$, and the proof is complete.

In the following lemma $\phi$ will denote a function on $X_{A}$ which is either

(i) $\phi(x)=y^{*}(x)$ for some non-negative functional $y^{*}$ or

(ii) $\phi(x)=\|x\|$.

In fact we may consider any real valued bounded sublinear function $\phi$ on $X_{A}$ such that for any two segments $d_{1}, d_{2}$ of $\mathfrak{D}$ with $d_{1} \subset d_{2}$ we have that $0 \leq \phi\left(x_{d_{1}}\right) \leq$ $\phi\left(x_{d_{2}}\right)$, where $d$ is a segment of $\mathfrak{D}$. Here, by $x_{d}$ we denote the vector $\sum_{\delta \in d} a_{\delta} e_{\delta}$.

Lemma 4.2. Let $E$ be a subset of $\mathfrak{D}$ of the form $E=\{\delta \in \mathfrak{D}: m \leq|\delta| \leq M\}, \phi$ a function on $X_{A}$ as before and $\varepsilon>0$. There exists a decomposition of $E$ into two disjoint subsets $E^{\prime}, E^{\prime \prime}$ such that

(1) $\left|\phi\left(E^{\prime} w\right)\right|<\varepsilon$ for every $w \in W_{0}$, and

(2) if $d=\left[\delta_{1}, \delta_{2}\right]$ is a segment of the tree such that $\left|\delta_{1}\right| \leq m,\left|\delta_{2}\right| \geq M$ and $d \cap E^{\prime \prime} \neq \emptyset$, then $\left|\phi\left(E x_{d}\right)\right| \geq \varepsilon$.

Proof. For every $\delta \in E$ let $\operatorname{ext}_{E}(\delta)=\operatorname{ext}(\delta) \cap E=\left[\delta^{E}, \delta\right]$, where $\delta^{E}$ is the unique element of $E$ such that $\delta^{E} \prec \delta$ and $\left|\delta^{E}\right|=m$. We set

$$
E^{\prime \prime}=\left\{\delta \in E:\left\|x_{\operatorname{ext}(\delta)}\right\| \leq 1 \text { and } \phi\left(x_{e^{x t_{E}}(\delta)}\right) \geq \varepsilon\right\}, E^{\prime}=E \backslash E^{\prime \prime} .
$$

Let $w \in W_{0}$; then $E w$ can be represented as $E w=\sum_{d \in L} \lambda_{d} x_{d}$, where $L$ is a subset of segments of $E$ such that $\left\|x_{e x t(d)}\right\| \leq 1$ for every $d \in L$ and $\sum_{d \in L}\left|\lambda_{d}\right| \leq 1$. For every $d \in L$ the set $d^{\prime}=d \cap E^{\prime}$ is either empty or a segment of $E$ and $\phi\left(x_{d^{\prime}}\right)<\varepsilon$. So $\left|\phi\left(E^{\prime} w\right)\right| \leq \sum_{d \in L}\left|\lambda_{d}\right|\left|\phi\left(x_{d^{\prime}}\right)\right|<\varepsilon$, which proves property (1). Property (2) of $E^{\prime \prime}$ is obvious.

The following two propositions are of combinatorial nature and we will use them to show that the set $W$ is an a-thin set. Both are related to the existence of incomparably supported elements of the set $W$. The first (Proposition 4.4) concerns finite families and the second (Proposition 4.6) is the infinite analog of Proposition 4.4. We begin with the following lemma. 
Lemma 4.3. Let $(\Omega, \mu)$ be a measure space with $\mu(\Omega) \leq 1$ and let $B_{1}, \ldots, B_{N}$ be measurable subsets of $\Omega$ satisfying $\mu\left(B_{i}\right) \geq \varepsilon$ for $i=1, \ldots, N$. Then for $k<N \varepsilon$ there exist $1 \leq i_{1}<\ldots<i_{k} \leq N$ such that $\bigcap_{j=1}^{k} B_{i_{j}} \neq \emptyset$.

Proof. If not, then the function $\sum_{i=1}^{N} \chi_{B_{i}}$ is bounded by $k$ on $\Omega$ and therefore $N \varepsilon \leq$ $\int_{\Omega}\left(\sum_{i=1}^{N} \chi_{B_{i}}\right) d \mu \leq k$, a contradiction.

Next we prove the first incomparability result related to finite families of elements of the set $W$.

Proposition 4.4. Let $\left(E_{i}\right)_{i=1}^{n}$ be a finite sequence of subsets of $\mathfrak{D}$ of the form $E_{i}=\left\{\delta \in \mathfrak{D}: m_{i} \leq|\delta| \leq M_{i}\right\}, m_{i}, M_{i} \in \mathbb{N}, M_{i}<m_{i+1}$ for $i=1, \ldots, n-1$. Then for every $\varepsilon>0, x^{*} \in B_{X_{A}^{*}}^{+}$and $\left(w_{i}\right)_{i=1}^{n}$ a finite sequence of vectors of $W_{0}$ there exists a partition $D_{1}, \ldots, D_{N}$ of $\{1,2, \ldots, n\}$ and $F_{i} \subset E_{i}$ for $i=1, \ldots, n$ such that

(1) If $r, s$ belong to the same set $D_{i}$ and $r<s$, then $\operatorname{ext}(\delta) \cap F_{r}=\emptyset$ for every $\delta \in F_{s}$ (i.e. the sets $\left(F_{r}\right)_{r \in D_{i}}$ are pairwise incomparable for every $\left.i=1, \ldots, N\right)$.

(2) $x^{*}\left(\left(E_{i} \backslash F_{i}\right) w_{i}\right)<\varepsilon$ for every $i=1, \ldots, n$.

(3) The number $N$ of the members of the partition is less than $\frac{5}{\varepsilon^{2}}$.

Proof. Applying Lemma 4.2 we find for every $i=1, \ldots, n$ a decomposition of $E_{i}$ into two disjoint subsets $E_{i}^{\prime}, E_{i}^{\prime \prime}$ such that

(a) $\left|x^{*}\left(E_{i}^{\prime} w_{i}\right)\right|<\frac{\varepsilon}{2}$.

(b) If $d=\left[\delta_{1}, \delta_{2}\right]$ is a segment such that $\left|\delta_{1}\right| \leq m_{i},\left|\delta_{2}\right| \geq M_{i}$ and $d \cap E_{i}^{\prime \prime} \neq \emptyset$, then $x^{*}\left(E_{i} x_{d}\right)>\frac{\varepsilon}{2}$.

For every $i=1,2, \ldots, n$ the vector $E_{i} w_{i}$ has a representation $E_{i} w_{i}=\sum_{d \in L_{i}} \lambda_{d} x_{d}$, where $L_{i}$ is a set of segments of $E_{i}$ with $\left\|x_{e x t(d)}\right\| \leq 1$ for every $d \in L_{i}$ and $\sum_{d \in L_{i}}\left|\lambda_{d}\right| \leq 1$

This representation defines a positive measure $\mu_{i}$ on the powerset of $\operatorname{seg}\left(E_{i}\right)=$ $\left\{d: d\right.$ is a segment of $E_{i}$ and $\left.\left\|x_{e x t(d)}\right\| \leq 1\right\}$, with $\left\|\mu_{i}\right\| \leq 1$, as follows: $\mu_{i}(A)=$ $\sum_{d \in A \cap L_{i}}\left|\lambda_{d}\right|$, for every $A \subset \operatorname{seg}\left(E_{i}\right)$.

For every $S \subset\{1, \ldots, n\}$ and every $i \in\{1, \ldots, n\}$ we set

$$
A_{S}^{i}=\left\{d \in \operatorname{seg}\left(E_{i}\right): \operatorname{ext}(d) \cap\left(\bigcup_{s \in S \backslash\{i\}} E_{s}^{\prime \prime}\right) \neq \emptyset\right\} .
$$

Given any non-empty subset $J$ of $\{1, \ldots, n\}$ we inductively define a non-empty subset $D(J)$ of $J$ in the following manner:

Suppose that $J=\left\{i_{1}<\ldots<i_{m}\right\}$. We let $i_{1}=\min J \in D(J)$. If $2 \leq k \leq m$ and we have selected all the elements among $\left\{i_{1}, \ldots, i_{k-1}\right\}$ that belong to $D(J)$, and denote them by $\left\{j_{1}, \ldots, j_{r}\right\}$ (so that in particular $j_{1}=i_{1}$ ), then $i_{k} \in D(J)$ if and only if $\mu_{i_{k}}\left(A_{\left\{j_{1}, \ldots, j_{r}\right\}}^{i_{k}}\right)<\frac{\varepsilon}{2}$.

We also set $F_{j}=\left\{\delta \in E_{j}^{\prime \prime}: \operatorname{ext}(\delta) \cap \bigcup\left\{E_{s}^{\prime \prime}: s<j, s \in D(J)\right\}=\emptyset\right\}$, for every $j \in D(J)$.

Observe that the sets $\left(F_{j}\right)_{j \in D(J)}$ are pairwise incomparable by definition. We show that $\left|x^{*}\left(E_{j} \backslash F_{j}\right) w_{j}\right|<\varepsilon$ for every $j \in D(J)$. Indeed, for $d \in L_{j}$ we set 
$d^{\prime}=E_{j}^{\prime} \cap d, d_{a}^{\prime \prime}=F_{j} \cap d$ and $d_{b}^{\prime \prime}=\left(E_{j}^{\prime \prime} \backslash F_{j}\right) \cap d$. It is clear that $d_{b}^{\prime \prime} \in A_{D(J)}^{j}$ for every $d \in L_{j}$, and since $\mu_{j}\left(A_{D(J)}^{j}\right)<\frac{\varepsilon}{2}$ we get that $\left|x^{*}\left(\left(E_{j} \backslash F_{j}\right) w_{j}\right)\right| \leq\left|x^{*}\left(E_{j}^{\prime} w_{j}\right)\right|+$ $\left|x^{*}\left(\sum_{d \in L_{j}} \lambda_{d} x_{d_{b}^{\prime \prime}}\right)\right| \leq \frac{\varepsilon}{2}+\sum_{d \in A_{D(J)}^{j}}\left|\lambda_{d}\right|<\varepsilon$. We define the partition $D_{1}, \ldots, D_{N}$ of $\{1,2, \ldots, n\}$ as follows: $J_{1}=\{1, \ldots, n\}, D_{1}=D\left(J_{1}\right), J_{2}=J_{1} \backslash D_{1}, D_{2}=D\left(J_{2}\right)$ and we continue this process until the set $J_{N+1}$ equals the empty set.

It remains to show that $N<\frac{5}{\varepsilon^{2}}$.

Indeed, choose $r \in J_{N}$. Then $\mu_{r}\left(A_{D_{j}}^{r}\right) \geq \frac{\varepsilon}{2}$ for $j=1, \ldots, N-1$.

If $N>\frac{5}{\varepsilon^{2}}$, then $(N-1) \frac{\varepsilon}{2}>\frac{2}{\varepsilon}$. By Lemma 4.3 we can select $D_{j_{1}}, \ldots, D_{j_{k}}$ with $j_{1}<\ldots<j_{k}$ and $\bigcap_{s=1}^{k} A_{D_{j_{s}}}^{r} \neq \emptyset$ for some $k$ such that $\frac{\varepsilon}{2} k>1$. Let $d \in \bigcap_{s=1}^{k} A_{D_{j_{s}}}^{r}$. Then for every $s=1, \ldots, k$ there exists $r_{s} \in D_{j_{s}}$ such that $d \cap E_{r_{s}}^{\prime \prime} \neq \emptyset$ so $x^{*}\left(E_{r_{s}} x_{e x t(d)}\right) \geq \frac{\varepsilon}{2}$ and $x^{*}\left(x_{e x t(d)}\right) \geq \frac{\varepsilon}{2} k>1$ which leads to a contradiction since $\left\|x^{*}\right\| \leq 1$.

Before passing to the proof of the infinite analog of Proposition 4.4 we give the statement and the proof of a known auxiliary result.

Lemma 4.5. Let $(\Omega, \mu)$ be a finite measure space, $\varepsilon>0$ and $\left(B_{n}\right)_{n=1}^{\infty}$ a sequence of measurable subsets of $\Omega$ such that $\mu\left(B_{n}\right) \geq \varepsilon$ for every $n \in \mathbb{N}$. There exists an infinite subset $M$ of $\mathbb{N}$ such that $\bigcap_{n \in M} B_{n} \neq \emptyset$.

Proof. Since $\mu(\Omega)<\infty$, it follows that

$$
\mu\left(\limsup B_{n}\right)=\mu\left(\bigcap_{n=1}^{\infty} \bigcup_{m=n}^{\infty} B_{n}\right) \geq \liminf \mu\left(B_{n}\right) \geq \varepsilon .
$$

Hence $\lim \sup B_{n} \neq \emptyset$ and for $\omega \in \lim \sup B_{n}$ there exists an infinite subset $M$ of $\mathbb{N}$ such that $\omega \in \bigcap_{n \in M} B_{n}$.

Proposition 4.6. Let $\left(E_{n}\right)_{n=1}^{\infty}$ be a sequence of subsets of $\mathfrak{D}$ of the form $E_{n}=$ $\left\{\delta \in \mathfrak{D}: m_{n} \leq|\delta| \leq M_{n}\right\}$, with $m_{n+1}>M_{n}$, for every $n \in \mathbb{N}$. Then for every sequence $\left(w_{n}\right)_{n=1}^{\infty}$ of vectors in $W_{0}$ and $\varepsilon>0$ there exist an infinite subset $I$ of $\mathbb{N}$ and sets $\left(F_{n}\right)_{n \in I}$ with $F_{n} \subset E_{n}$, for all $n \in I$, so that:

(1) The sets $\left(F_{n}\right)_{n \in I}$ are pairwise incomparable.

(2) For every $n \in I,\left\|\left(E_{n} \backslash F_{n}\right) w_{n}\right\|<\varepsilon$.

Proof. We apply Lemma 4.2 to find a decomposition of each $E_{n}$ into two disjoint sets $E_{n}^{\prime}, E_{n}^{\prime \prime}$ such that

(1) $\left\|E_{n}^{\prime} w_{n}\right\|<\frac{\varepsilon}{2}$.

(2) If $d=\left[\delta_{1}, \delta_{2}\right]$ is a segment with $\left|\delta_{1}\right| \leq m_{n},\left|\delta_{2}\right| \geq M_{n}$ and $d \cap E_{n}^{\prime \prime} \neq \emptyset$, then $\left\|E_{n} x_{d}\right\| \geq \frac{\varepsilon}{2}$.

For every $n \in \mathbb{N}$ the vector $E_{n} w_{n}$ has a representation as $E_{n} w_{n}=\sum_{d \in L_{n}} \lambda_{d} x_{d}$, where $L_{n} \subseteq \operatorname{seg}\left(E_{n}\right)=\left\{d: d \subset E_{n}\right.$ and $\left.\left\|x_{e x t(d)}\right\| \leq 1\right\}$ and $\sum_{d \in L_{n}}\left|\lambda_{d}\right| \leq 1$.

This representation defines a positive measure $\mu_{n}$ on $\operatorname{seg}\left(E_{n}\right)$ as in the previous proposition: $\mu_{n}(A)=\sum_{d \in A \cap L_{n}}\left|\lambda_{d}\right|$, for every $A \subset \operatorname{seg}\left(E_{n}\right)$.

Consider any probability measure $\nu$ on the compact metrisable space $\Gamma$ of all infinite branches of $\mathfrak{D}$ such that $\nu\left(W_{d}\right) \neq \emptyset$ for every $d$ segment of the tree, where 
$W_{d}$ is the basic clopen subset of $\Gamma$ consisting of all branches that contain $d$ (for instance $\nu$ is the natural measure defined by $\nu\left(W_{\text {ext }(\delta)}\right)=\frac{1}{k_{|\delta|}}$ for every $\delta \in \mathfrak{D}$, where $\left.k_{n}=\#\{\delta \in \mathfrak{D}:|\delta|=n\}\right)$. We define a measure $\mu$ on $\Gamma$ as follows: For every clopen subset $B$ of $\Gamma, \mu(B)=\lim _{n \rightarrow \mathfrak{U}_{d \in L_{n}}} \sum_{d}\left|\lambda_{d}\right| \frac{\nu\left(W_{d} \cap B\right)}{\nu\left(W_{d}\right)}$, where the limit is taken with respect to some non-trivial ultrafilter $\mathfrak{U}$ on $\mathbb{N}$.

Using a diagonal argument we may actually assume that this limit is an ordinary limit, by passing to an appropriate subsequence. We shall also assume, without loss of generality, that this subsequence is the whole sequence of natural numbers.

For every $n, s \in \mathbb{N}$ with $n<s$ we define $B_{n}=\left\{\gamma \in \Gamma: \gamma \cap E_{n}^{\prime \prime} \neq \emptyset\right\}, A_{n}^{s}=\{d \in$ $\left.\operatorname{seg}\left(E_{s}\right): \operatorname{ext}(d) \cap E_{n}^{\prime \prime} \neq \emptyset\right\}$.

It is easy to check that for every $n \in \mathbb{N}, \mu\left(B_{n}\right)=\lim _{s \rightarrow \infty} \mu_{s}\left(A_{n}^{s}\right)$.

Claim. For every infinite subset $I$ of $\mathbb{N}$ and $\theta>0$ the set $I_{\theta}=\left\{n \in I: \mu\left(B_{n}\right)<\theta\right\}$ is infinite.

Indeed, otherwise there exists an infinite subset $J$ of $I$ such that $\mu\left(B_{n}\right) \geq \theta$ for every $n \in J$ and by Lemma 4.5 there exists an infinite subset $J^{\prime}$ of $J$ and $\gamma \in \bigcap_{n \in J^{\prime}} B_{n}$. For every $n \in J^{\prime}, \gamma \cap E_{n}^{\prime \prime} \neq \emptyset$ and so $\left\|E_{n}^{\prime \prime} x_{\gamma}\right\| \geq \frac{\varepsilon}{2}$ for infinitely many values of $n$. Select $\delta_{n} \in E_{n}^{\prime \prime} \cap \gamma$. Then $\left\|x_{\operatorname{ext}\left(\delta_{n}\right)}\right\| \leq 1$ and so $\left\|x_{\gamma}\right\| \leq 1$ which contradicts the fact that the basis of $X_{A}$ is boundedly complete.

We define $I_{0}=\mathbb{N}$ and inductively for $k \geq 0$,

$$
\begin{gathered}
\tilde{I}_{k+1}=\left\{n \in I_{k}: \mu\left(B_{n}\right)<\frac{\varepsilon}{2^{k+2}}\right\}, \quad n_{k+1}=\min \tilde{I}_{k+1}, \\
I_{k+1}=\left\{s \in \tilde{I}_{k+1}: \mu_{n_{k+1}}\left(A_{n_{k+1}}^{s}\right)<\frac{\varepsilon}{2^{k+2}}\right\}
\end{gathered}
$$

and

$$
F_{n_{k+1}}=\left\{\delta \in E_{n_{k+1}}^{\prime \prime}: \operatorname{ext}(\delta) \cap\left(\bigcup_{i=1}^{k} E_{n_{i}}^{\prime \prime}\right)=\emptyset\right\} .
$$

By the previous claim the sets $\tilde{I}_{k+1}, I_{k+1}$ are infinite subsets of $I_{k}$ and since $n_{k+1} \in I_{k}, \mu_{n_{k+1}}\left(A_{n_{k}}^{n_{k+1}}\right)<\frac{\varepsilon}{2^{k+1}}$.

We set $I=\left\{n_{1}, n_{2}, \ldots\right\}$ and we observe that the sets $\left(F_{n_{j}}\right)_{j=1}^{\infty}$ are pairwise incomparable by their own definition. We show that for every $k,\left\|\left(E_{n_{k}} \backslash F_{n_{k}}\right) w_{n_{k}}\right\|<$ $\varepsilon$. If $k=1$ this follows from the fact that $F_{n_{1}}=E_{n_{1}}^{\prime \prime}$.

Let $k \in \mathbb{N}$ and set $r=n_{k+1}$. Consider the set: $A_{r}=A_{n_{1}}^{r} \cup \ldots \cup A_{n_{k}}^{r}$. Then, $\mu_{r}\left(A_{r}\right) \leq \frac{\varepsilon}{2^{2}}+\ldots+\frac{\varepsilon}{2^{k+1}}<\frac{\varepsilon}{2}$. Let $d \notin A_{r}$; then for every $\delta \in d$ and $i=1, \ldots, k$, $\operatorname{ext}(\delta) \cap E_{n_{i}}^{\prime \prime}=\emptyset$ so $d \subset F_{r}$. Conversely if $d \in A_{r}$, then $d \cap F_{r}=\emptyset$. So $\left\|\left(E_{r}^{\prime \prime} \backslash F_{r}\right) w_{r}\right\| \leq \sum_{d \in L_{s} \cap A_{r}}\left|\lambda_{d}\right|\left\|\left(E_{r}^{\prime \prime} \backslash F_{r}\right) x_{d}\right\| \leq \mu_{r}\left(A_{r}\right)<\frac{\varepsilon}{2}$.

Finally, we have $\left\|\left(E_{r} \backslash F_{r}\right) w_{r}\right\| \leq\left\|\left(E_{r}^{\prime}\right) w_{r}\right\|+\left\|\left(E_{r}^{\prime \prime} \backslash F_{r}\right) w_{r}\right\|<\frac{\varepsilon}{2}+\frac{\varepsilon}{2}=\varepsilon$.

The present form of Propositions 4.4 and 4.6 is a variation of our original results suggested by B. Maurey.

Definition 4.1. Let $\left(A,\|\|_{A}\right)$ be a reflexive Banach space with an unconditional basis $\left(e_{n}\right)_{n=1}^{\infty}$, and let $\left(C_{k}\right)_{k=1}^{\infty}$ be a sequence of positive real numbers. We say that $A$ satisfies property $(P)$ for the sequence $\left(C_{k}\right)_{k=1}^{\infty}$ if for every block sequence $\left(x_{n}\right)_{n=1}^{\infty}$ of $\left(e_{n}\right)_{n=1}^{\infty}$ and every $k \in \mathbb{N}$ there exists a normalized block sequence 
$\left(y_{n}\right)_{n=1}^{\infty}$ of $\left(x_{n}\right)_{n=1}^{\infty}$ satisfying the following condition: For every infinite subset $M$ of $\mathbb{N}$ there exist $E \subset M$ and $\left(\lambda_{n}\right)_{n \in E} \subseteq \mathbb{R}^{+},\left(y_{n}^{*}\right)_{n \in E} \subseteq B_{A^{*}}$ such that

(i) $\sum_{n \in E} \lambda_{n}=1,\left\|\sum_{n \in E} \lambda_{n} y_{n}\right\|_{A}<\frac{1}{k}$,

(ii) $\operatorname{suppy}_{n}^{*} \subseteq \operatorname{suppy}_{n}$ and $y_{n}^{*}\left(y_{n}\right)>\frac{1}{2}$ for every $n \in E$ and

(iii) for every $x \in B_{A},\left\|\sum_{n \in E} y_{n}^{*}(x) y_{n}\right\|_{A} \leqslant C_{k}$.

Remark 4.1. Condition (iii) in the above definition is equivalent to the fact that the norm of the operator $P: X \rightarrow \overline{\left\langle\left(y_{n}\right)_{n \in E}\right\rangle}$ defined by the relation $P(x)=\sum_{n \in E} y_{n}^{*}(x) y_{n}$ is dominated by $C_{k}$. There are other conditions which imply condition (iii) and in some cases they are more convenient to be checked. We give two of them for later use:

$\left(\bar{P}_{1}\right):\left\|\sum_{n \in E} y_{n}^{*}\right\| \leq C_{k}, \quad\left(\bar{P}_{2}\right):|E| \leq C_{k}$.

In the sequel by property $\left(P_{i}\right), i=1,2$, we will mean property $(P)$ where condition (iii) has been replaced by $\left(P_{i}\right), i=1,2$.

To see that $\left(P_{i}\right), i=1,2$, imply $(P)$ we observe that $\left(P_{2}\right) \Rightarrow\left(P_{1}\right)$ is obvious, while the implication $\left(P_{1}\right) \Rightarrow(P)$ follows from the unconditionality of the basis $\left(e_{n}\right)_{n}$.

Lemma 4.7. If the Banach space $\left(A,\|\|_{A}\right)$ satisfies property $(P)$ for the sequence $\left(C_{k}\right)_{k=1}^{\infty}$, then the same remains valid for the space $\left(X_{A},\|\|\right)$ and the same sequence $\left(C_{k}\right)_{k=1}^{\infty}$.

Proof. For every $x=\sum_{\delta \in \mathfrak{D}} \lambda_{\delta} e_{\delta}$ we set $\bar{x}=\sum_{i=1}^{\infty}\left(\sum_{|\delta|=i}\left|\lambda_{\delta}\right|\right) e_{i}$. It follows from the definition of the space $X_{A}$ that $\|x\|=\|\bar{x}\|$.

Let $\left(x_{n}\right)_{n=1}^{\infty}=\left(\sum_{|\delta| \in E_{n}} \lambda_{\delta} e_{\delta}\right)_{n \in \mathbb{N}}$ be a block sequence in $X_{A}$. By passing to a subsequence of $\left(x_{n}\right)_{n=1}^{\infty}$, if necessary, we may assume that $E_{1}<E_{2}<\ldots$ are successive subsets of $\mathbb{N}$ and so the corresponding $\left(\bar{x}_{n}\right)_{n=1}^{\infty}$ is a block sequence in $A$ such that $\left\|x_{n}\right\|=\left\|\bar{x}_{n}\right\|_{A}$.

Since the space $A$ satisfies property $(P)$ it follows that for the sequence $\left(\bar{x}_{n}\right)_{n=1}^{\infty}$ and a given $k>0$ there exists a normalized block $\left(\bar{y}_{n}\right)_{n=1}^{\infty}$ of $\left(\bar{x}_{n}\right)$ such that conditions (i), (ii), (iii) of Definition 4.1 are fulfilled.

Each $\bar{y}_{n}=\sum_{i \in F_{n}} \lambda_{i} \bar{x}_{i}$ with $F_{1}<F_{2}<\ldots$. We set $y_{n}=\sum_{i \in F_{n}} \lambda_{i} x_{i}$ which clearly is a normalized block subsequence of $\left(x_{n}\right)_{n=1}^{\infty}$ in the space $X_{A}$. Let $M$ be an infinite subset of $\mathbb{N}$. By our assumptions there exist $E \subset M,\left(\theta_{n}\right)_{n \in E} \subset \mathbb{R}^{+}$, and $\left(\bar{y}_{n}^{*}\right)_{n \in E} \subset B_{A^{*}}$ such that

(a) $\sum_{n \in E} \theta_{n}=1$ and $\left\|\sum_{n \in E} \theta_{n} \bar{y}_{n}\right\|_{A}<\frac{1}{k}$.

(b) For all $n \in E$, supp $\bar{y}_{n}^{*} \subset \operatorname{supp} \bar{y}_{n}$ and $\bar{y}_{n}^{*}\left(\bar{y}_{n}\right)>\frac{1}{2}$.

(c) For every $x \in B_{A},\left\|\sum_{n \in E} \bar{y}_{n}^{*}(x) \bar{y}_{n}\right\|_{A} \leqslant C_{k}$. 
Since $\operatorname{supp} \bar{y}_{n}^{*} \subset \operatorname{supp} \bar{y}_{n}$ it follows that $\bar{y}_{n}^{*}=\sum_{i \in F_{n}}\left(\sum_{j \in E_{i}} \alpha_{j} e_{j}^{*}\right)$. We define $y_{n}^{*}=$ $\sum_{i \in F_{n}} \sum_{j \in E_{i}} \sum_{|\delta|=j}\left(\operatorname{sgn} \lambda_{\delta}\right) \alpha_{j} e_{\delta}^{*}$, where $\operatorname{sgn} \lambda_{\delta}=0$ if $\lambda_{\delta}=0$ and $\lambda_{\delta}\left(\operatorname{sgn} \lambda_{\delta}\right)=\left|\lambda_{\delta}\right|$ otherwise. It is easily checked that $\left(y_{n}\right)_{n \in E},\left(y_{n}^{*}\right)_{n \in E},\left(\theta_{n}\right)_{n \in E}$ satisfy the conditions corresponding to (a), (b), (c) in the space $X_{A}$ and this completes the proof.

Remark 4.2. It can be shown that the analogous results for properties $\left(P_{i}\right), i=1,2$, also hold. The proof of them follows similar steps and is left to the reader.

The following result is well known $[\mathrm{N}]$ and says that if a closed set almost absorbs the unit ball $B_{X}$ of the whole space, then it actually absorbs $B_{X}$. We will state it in the following form:

Lemma 4.8. Let $W$ be a convex subset of a Banach space $X$ such that $B_{X} \subseteq$ $\lambda W+\varepsilon B_{X}$, for some $\lambda>0$ and $\varepsilon<1$. Then $B_{X} \subset \frac{\lambda}{1-\varepsilon} \bar{W}$.

Theorem 4.9. Let $\left(A,\|\|_{A}\right)$ be a reflexive Banach space with an unconditional basis which satisfies property $(P)$ for a sequence $\left(C_{k}\right)_{k=1}^{\infty}$. We set $\mathbf{a}=\left(a_{n}\right)_{n=1}^{\infty}=$ $\left(\frac{1}{n C_{n 2^{n}}}\right)_{n=1}^{\infty}$. Then the set $W$ is an $\mathbf{a}$-thin subset of the space $X_{A}$.

Proof. Suppose that $W$ is not an a-thin subset of $X_{A}$. Then there exists a normalized sequence $\left(x_{n}\right)_{n=1}^{\infty}$ of successive blocks of $\left(e_{\delta}\right)_{\delta \in \mathfrak{D}}$ and $\lambda>0$ such that for every $n \in \mathbb{N}$,

$$
B_{X} \subseteq \lambda\left(2^{n} W+a_{n} B_{X_{A}}\right)
$$

where $X=\overline{\operatorname{span}}\left(x_{n}\right)_{n=1}^{\infty}$. Fix $k \in \mathbb{N}$ with $k>16 \lambda$.

The space $X_{A}$ also satisfies property $(P)$ for the same sequence $\left(C_{k}\right)_{k}$, by Lemma 4.7. Hence there exists a normalized block sequence $\left(y_{n}\right)_{n=1}^{\infty}$ of $\left(x_{n}\right)_{n=1}^{\infty}$ such that for every infinite subset $M$ of $\mathbb{N}$ we can find $E \subset M,\left(\theta_{n}\right)_{n \in E} \subseteq \mathbb{R}^{+},\left(y_{n}^{*}\right)_{n \in E} \subseteq B_{X_{A}^{*}}$ such that

$$
\begin{aligned}
& \sum_{n \in E} \theta_{n}=1, \quad\left\|\sum_{n \in E} \theta_{n} y_{n}\right\|<\frac{1}{k 2^{k}}, \\
& \operatorname{supp}_{n}^{*} \subset \operatorname{supp}_{n}, \quad y_{n}^{*}\left(y_{n}\right)>\frac{1}{2}
\end{aligned}
$$

and

$$
\|P\| \leqslant C_{k 2^{k}}
$$

where $P(x)=\sum_{n \in E} y_{n}^{*}(x) y_{n}$.

Let $w_{n} \in W$ be such that $\left\|y_{n}-\lambda 2^{k} w_{n}\right\|<\lambda a_{k}$ and set $E_{n}=s u p p y_{n}$.

By Proposition 4.6 there exists an infinite subset $M$ of $\mathbb{N}$ and a family $\left(F_{n}\right)_{n \in M}$ of pairwise incomparable subsets of $\mathfrak{D}$ such that for every $n \in \mathbb{N}$

$$
F_{n} \subset E_{n} \text { and }\left\|E_{n} w_{n}-F_{n} w_{n}\right\|<\frac{a_{k}}{2^{k} \lambda} .
$$

Let $E \subset M,\left(\theta_{n}\right)_{n \in E} \subset \mathbb{R}^{+},\left(y_{n}^{*}\right)_{n \in E} \subset B_{X_{A}^{*}}$ satisfy the above conditions and set $z_{n}^{*}=\left.y_{n}^{*}\right|_{F_{n}}, R(x)=\sum_{n \in E} z_{n}^{*}(x) y_{n}$.

Since the basis of $X_{A}$ is 1-unconditional it follows that

$$
\|R\| \leq\|P\| \leq C_{k 2^{k}}
$$


and

$$
\left|z_{n}^{*}\left(y_{n}\right)\right|>\frac{1}{4}
$$

Set $Y_{E}=\operatorname{span}\left[\left(y_{n}\right)_{n \in E}\right]$. Then (1) yields that

$$
R\left(B_{Y_{E}}\right) \subset \lambda 2^{k} R(W)+\lambda a_{k} R\left(B_{X_{A}}\right)
$$

and by (5) we get that $\lambda a_{k} R\left(B_{X_{A}}\right) \subset \lambda a_{k} C_{k 2^{k}} B_{Y_{E}} \subset \frac{\lambda}{\kappa} B_{Y_{E}} \subset \frac{1}{16} B_{Y_{E}}$.

Also (6) implies that $\frac{1}{4} B_{Y_{E}} \subset R\left(B_{Y_{E}}\right)$. Substituting in (7) we obtain that $B_{Y_{E}} \subset 4 \lambda 2^{k} R(W)+\frac{1}{4} B_{Y_{E}}$ and from Lemma 4.8 we deduce that

$$
B_{Y_{E}} \subset 8 \lambda 2^{k} R(W) .
$$

Since the sets $\left(\operatorname{supp} z_{n}^{*}\right)_{n \in E}$ are pairwise incomparable we get that $R(W) \subset$ $c o\left[\left( \pm y_{i}\right)_{i \in E}\right]$.

Therefore $B_{Y_{E}} \subset 8 \lambda 2^{k} c o\left[\left( \pm y_{i}\right)_{i \in E}\right]$ and since $k 2^{k}\left(\sum_{n \in E} \theta_{n} y_{n}\right) \in B_{Y_{E}}$ we conclude that $k \leq 8 \lambda$, which derives a contradiction since we had selected $k>16 \lambda$.

Remark 4.3. Assuming that the sequence $\left(C_{k}\right)_{k=1}^{\infty}$ in the above theorem is bounded or even if $C_{k} \leq C k$ and $\left(P_{1}\right)$ holds, then our arguments can show that the set $W$ is actually a thin subset of $X_{A}$.

Next we state the following well known lemma (cf. [LT], 1c8).

Lemma 4.10. Let $A$ be a reflexive Banach space with an unconditional basis and $P: A \rightarrow Y$ a bounded projection of $A$ onto a block subspace $Y$ of $A$ spanned by a normalized block sequence $\left(y_{n}\right)_{n=1}^{\infty}$. Then there exists a bounded projection $R: A \rightarrow Y$ of the form $R(x)=\sum_{n=1}^{\infty} y_{n}^{*}(x) y_{n}$, where $\left(y_{n}^{*}\right)_{n=1}^{\infty}$ is a block sequence in $A^{*}$ with $\operatorname{supp}\left(y_{n}^{*}\right) \subset \operatorname{supp}\left(y_{n}\right)$ for every $n \in \mathbb{N}$.

Remark 4.4. It is easily seen that if a reflexive Banach space $A$ satisfies the complemented subspace property described in the statement of Lemma 4.10, then the same property holds for the space $X_{A}$.

The following result has been proved by N.Tomczak-Jaegermann who kindly permitted us to include it here.

Theorem 4.11. Let $A$ be a reflexive Banach space with an unconditional basis such that every block subspace of $A$ has a block subspace complemented in $A$. Then the set $W$ is a thin subset of $X_{A}$.

Proof. We recall that the basis of $A$, and therefore the basis of $X_{A}$, is as well bimonotone.

Suppose that the set $W$ is not a thin subset of $X_{A}$. Then there exists a normalized block sequence $\left(x_{n}\right)_{n}$ of $X_{A}$ so that letting $X=\overline{\operatorname{span}}\left[\left(x_{n}\right)_{n}\right]$ we have that for every $\varepsilon>0$ there exists a $\lambda>0$ such that $B_{X} \subset \lambda W+\varepsilon B_{X_{A}}$.

Lemma 4.10 and Remark 4.4 allow us to assume (by passing to a normalized block sequence of $\left(x_{n}\right)_{n}$, if necessary) that there exists a sequence $\left(x_{n}^{*}\right)_{n} \subset X_{A}^{*}$, such that for every $n \in \mathbb{N}, \operatorname{supp}\left(x_{n}^{*}\right) \subset \operatorname{range}\left(x_{n}\right), x_{n}^{*}\left(x_{n}\right)=1$ and the operator $P(x)=\sum_{n=1}^{\infty} x_{n}^{*}(x) x_{n}$ is bounded. Since the basis of $X_{A}$ is bimonotone we have that $\left\|x_{n}^{*}\right\| \leq\|P\|$ for all $n$. Let $\varepsilon=\frac{1}{8\|P\|}$ and $\lambda>0$ such that $B_{X} \subset \lambda W+\varepsilon B_{X_{A}}$. 
For every $n \in \mathbb{N}$ we select $w_{n} \in W_{0}$ such that $\left\|x_{n}-\lambda E_{n} w_{n}\right\|<\varepsilon$, where $E_{n}=$ range $\left(x_{n}\right)$.

Applying Proposition 4.6 we obtain an infinite subset $I$ of $\mathbb{N}$ and $\left(F_{n}\right)_{n \in I}$ pairwise incomparable subsets of $\mathfrak{D}$ such that for every $n \in I, F_{n} \subset E_{n}$ and $\left\|E_{n} w_{n}-F_{n} w_{n}\right\|$

$<\frac{1}{4 \lambda}$. We set $y_{n}^{*}=F_{n} x_{n}^{*}$ and $R(x)=\sum_{n=1}^{\infty} y_{n}^{*}(x) x_{n}$.

We observe that $\|R\| \leq\|P\|$ and $\left|y_{n}^{*}\left(x_{n}\right)\right|>\frac{1}{4}$. Let $Y=\overline{\operatorname{span}}\left[\left(x_{n}\right)_{n \in I}\right]$. Since $\left|y_{n}^{*}\left(x_{n}\right)\right|>\frac{1}{4}$ we obtain that

$$
\frac{1}{4} B_{Y} \subset R\left(B_{Y}\right)
$$

Also since $\varepsilon=\frac{1}{8\|P\|}$ we have that

$$
R\left(B_{Y}\right) \subset \lambda R(W)+\frac{1}{8} B_{Y} .
$$

(1) and (2) and Lemma 4.8 yield that

$$
B_{Y} \subset 8 \lambda \overline{R(W)}
$$

Since the operator $R$ is defined by the sequence $\left(y_{n}^{*}\right)_{n \in I}$ and the sets $\left(\operatorname{supp} y_{n}^{*}\right)_{n \in I}$ are pairwise incomparable we have that $R(W) \subset\|P\| \overline{c o}\left[\left( \pm x_{n}\right)_{n \in I}\right]$, and finally

$$
B_{Y} \subset(8 \lambda\|P\|) \overline{c o}\left[\left( \pm x_{n}\right)_{n \in I}\right] .
$$

To derive a contradiction we select a convex combination $z=\sum_{n \in E} \theta_{n} x_{n}$ such that $\|z\|<\frac{1}{16 \lambda|| P||}$ and observe that $(16 \lambda\|P\|) z \in B_{Y}$, contradicting (3). The proof of the theorem is complete.

Remark 4.5. It is known that $\ell^{p}(\mathbb{N})$ where $1<p<\infty$, Tsirelson space and Schlumprecht space satisfy the assumptions of the above proposition. Therefore if $A$ is any of these spaces the set $W$ in $X_{A}$ is a thin set.

Definition 4.2. Let $X$ be a Banach space with a basis $\left(e_{n}\right)_{n=1}^{\infty}$ and $1<p \leq \infty$. We say that $X$ has an upper $p$-estimate if there exists $C>0$ such that for every normalized block sequence $\left(x_{i}\right)_{i=1}^{\infty}$ of $\left(e_{n}\right)_{n=1}^{\infty}$ we have that $\left\|\sum_{i=1}^{n} x_{i}\right\| \leq C n^{\frac{1}{p}}$.

Remark 4.6. If a space $X$ has an upper $p$-estimate for some $p>1$, then it satisfies property $\left(P_{2}\right)$ with $C_{k} \leq(C k)^{\frac{p}{p-1}}$, where $C$ is the constant in Definition 4.2 and hence it also satisfies property $(P)$ (see Remark 4.1). Spaces with an upper $p$ estimate are $L^{p}(\mu)$ where $1<p<\infty$, and in general are Banach spaces where $\ell^{1}$ is not uniformly block representable ([MS]). It follows that such a reflexive space with an unconditional basis has the property that the set $W$ in the space $X_{A}$ is a-thin for an appropriate sequence a depending on $p$.

We pass now to show that certain classes of operators consist of thin operators. As we have already pointed out, every thin operator is strictly singular but the converse is not always true. However, if we restrict our interest in the classes $\mathcal{L}\left(\ell^{p}, \ell^{r}\right), 1 \leq p, r<\infty$, then the subsets of strictly singular operators and thin operators coincide. This follows from the next lemmas:

Lemma 4.12. Let $T$ be in $\mathcal{L}\left(X, \ell^{p}\right)$ where $1 \leq p<\infty$, which is not a thin operator. Then there exist a block subspace $Z$ of $\ell^{p}$ and a projection $P: \ell^{p} \rightarrow Z$ with $\|P\|=1$ such that $P \circ T$ is a quotient map. 
Proof. Choose any $\varepsilon<1$. Then there exists an infinite-dimensional closed subspace $Z$ of $\ell^{p}$ and $\lambda \in \mathbb{R}^{+}$such that $B_{Z} \subset \lambda T\left[B_{X}\right]+\varepsilon B_{\ell^{p}}$. Further, we may assume that $Z$ is a block subspace of $\ell^{p}$ and hence there exists a projection $P: \ell^{p} \rightarrow Z$ with $\|P\|=1$. Therefore $B_{Z} \subset \lambda P\left(T\left[B_{X}\right]\right)+\varepsilon B_{Z}$ and from Lemma $4.8 B_{Z} \subset$ $\frac{\lambda}{1-\varepsilon} P \circ T\left[B_{X}\right]$ which implies that $P \circ T$ is a quotient map.

The proof of the following lemma is similar to the proof of the lifting property of $\ell^{1}$ (see [LT], p.107).

Lemma 4.13. If $T: \ell^{p} \rightarrow \ell^{p}$, where $1 \leq p<\infty$, is a bounded linear onto operator, then there exists an infinite-dimensional closed subspace $Z$ of $\ell^{p}$ such that $\left.T\right|_{Z}$ is an isomorphism.

Corollary 4.14. (a) If $1 \leq p \neq r<\infty$, then every $T \in \mathcal{L}\left(\ell^{p}, \ell^{r}\right)$ is a thin operator.

(b) If $1 \leq p<\infty$ and $T \in \mathcal{L}\left(\ell^{p}, \ell^{p}\right)$, then $T$ is a strictly singular operator if and only if it is a thin operator.

\section{Thin norming Sets II (General Reflexive Case)}

In this section we prove that every reflexive space $A$ with an unconditional basis has a subspace $B$ such that the set $W$ in $X_{B}$ is an a-thin set for an appropriate null sequence $\mathbf{a}$. The proof heavily depends on the uniform control on a subspace of $A$ of the rate of convergence to zero in norm of certain convex combinations. Therefore the ordinal rank of the complexity of weakly null sequences seems to be necessary and we strongly use the results of [AMT]. The main parts of this section are the following: The first part contains the definitions of Schreier families, initially introduced in [AA], and RA-Hierarchy from [AMT]. We also present some results from [AMT] that we will use here. The most important of them are the dichotomy principle (Proposition [5.3) and the large families theorem (Theorem 5.4). In the second part, for a countable ordinal $\xi$, a natural number $n$ and $B$ a subspace of $A$ we introduce the numerical quantity $\tau_{\xi, n}(B)$ which is basic for our proof. We show that there exists a countable ordinal $\xi_{0}$ such that $\tau_{\xi, n}(B)=0$ for every $\xi \geq \xi_{0}$ and $n \in \mathbb{N}$. Moreover, there exists a subspace $B$ of $A$ so that $\tau_{\xi, n}$ is stabilized over all further subspaces. This subspace $B$ is the desired subspace of $A$. To accomplish this, we need to show that for every $\varepsilon>0$ there exists $(\xi, n)$ such that $0<\tau_{\xi, n}(B)<\varepsilon$. Finally, in the last part (Propositions 5.18, 5.20, Theorem 5.21), we prove that the set $W$ in $X_{B}$ is an a-thin set.

Schreier families. By $[\mathbb{N}]\left(\right.$ resp. $[\mathbb{N}]^{<\omega}$ ) we will denote the set of all infinite (resp. finite) subsets of the set $\mathbb{N}$ of natural numbers. Also for $M \in[\mathbb{N}]$ we denote by $[M]$ the set of all infinite subsets of $M$.

For every countable ordinal $\xi$ we define a family $S_{\xi}$ of finite subsets of $\mathbb{N}$ as follows:

(1) $S_{0}=\{\{n\}: n \in \mathbb{N}\} \cup\{\emptyset\}$.

(2) If $S_{\xi}$ has been defined, then

$$
S_{\xi+1}=\left\{\bigcup_{i=1}^{n} F_{i}: n \in \mathbb{N}, n \leq F_{1}<F_{2}<\ldots<F_{n}, F_{i} \in S_{\xi}\right\} \cup\{\emptyset\} .
$$

(3) If $\xi$ is a limit ordinal and for every $\zeta<\xi$ the family $S_{\zeta}$ has been defined, then we fix a strictly increasing sequence $\left(\xi_{n}\right)_{n \in \mathbb{N}}$ of non-limit ordinals with $\sup _{n \in \mathbb{N}} \xi_{n}=\xi$ and define $S_{\xi}=\left\{F: n \leq \min F\right.$ and $F \in S_{\xi_{n}}$ for some $\left.n \in \mathbb{N}\right\} \cup\{\emptyset\}$. 
Notation. Let $M \in[\mathbb{N}]$, and let $\xi$ be a countable ordinal. We introduce two more families related to $S_{\xi}$ and the set $M$. If $M=\left(m_{k}\right)_{k \in \mathbb{N}}$ we set $S_{\xi}^{M}=\{G \subset M$ : there exists $F \in S_{\xi}$ such that $\left.G=\left\{m_{\ell}: \ell \in F\right\}\right\}$. We also denote $S_{\xi}[M]=\{F \subset$ $\left.M: F \in S_{\xi}\right\},\left[S_{\xi}^{M}\right]^{n}=\left\{\bigcup_{i=1}^{n} F_{i}: F_{i} \in S_{\xi}^{M}, F_{1}<F_{2}<\ldots<F_{n}\right\}$.

Definition 5.1. A family $\mathfrak{F}$ of subsets of $\mathbb{N}$ is said to be:

(a) hereditary if $G \in \mathfrak{F}$ for every $F \in \mathfrak{F}$ and $G \subset F$;

(b) adequate if it is hereditary and compact in the topology of pointwise convergence as a subspace of $\{0,1\}^{\mathbb{N}}$.

Properties of $\left(S_{\xi}\right)_{\xi<\omega_{1}}$.

(1) Each $S_{\xi}$ is an adequate family.

(2) Each $S_{\xi}$ is spreading, i.e. if $G=\left\{m_{i}\right\}_{i=1}^{d} \in S_{\xi}$ and $G^{\prime}=\left\{m_{i}^{\prime}\right\}_{i=1}^{d}$ is such that $m_{i} \leq m_{i}^{\prime}, i \leq d$, then $G^{\prime} \in S_{\xi}$.

(3) If $G \in S_{\xi}, \max G<\min \{m, n\}$, then $G \cup\{n\} \in S_{\xi}$ if and only if $G \cup\{m\} \in S_{\xi}$.

(4) If $L \in[\mathbb{N}], L=\left(l_{j}\right)_{j \in \mathbb{N}},\left\{l_{1}, \ldots, l_{d}\right\} \in S_{\xi}$ and $\left\{l_{1}, \ldots, l_{d}, l_{d+1}\right\} \notin S_{\xi}$, then $\left\{l_{1}, \ldots, l_{d}\right\}$ is a maximal member of $S_{\xi}$.

(5) If $\zeta<\xi$, then there exists $n(\zeta, \xi)=n \in \mathbb{N}$ such that $S_{\zeta}[N \backslash\{1, \ldots, n-1\}] \subset$ $S_{\xi}$.

(6) If $M \in[\mathbb{N}]$, then $S_{\xi}^{M} \subset S_{\xi}[M]$ but the converse is not always true.

(7) If $M \in[L]$, then $S_{\xi}[M] \subset S_{\xi}[L]$ and $S_{\xi}^{M} \subset S_{\xi}^{L}$.

Properties (1), (2) and (3) are proved by induction. Property (4) is a consequence of properties (2) and (3). Indeed, if $\left\{l_{1}, \ldots, l_{d}\right\} \cup\{m\} \in S_{\xi}$ for some $m \in \mathbb{N}$, then from (3) we get that $m<l_{d}$. Further from (2) we conclude that $\left\{l_{1}, \ldots, l_{d}, l_{d+1}\right\} \in S_{\xi}$, contradicting our assumption. Property (5) is also proved by induction. Property (6) follows from property (2), and property (7) is an easy consequence of the definitions.

The following lemma is due to G. Androulakis and T. Odell and shows that after passing to an appropriate subset $N$ of $M$ we have that the members of $S_{\xi}[N]$, $S_{\xi}^{M}[N]$ are almost equal.

Lemma 5.1 ([ब] $)$. For every $M \in[\mathbb{N}]$ there exists $N \in[M]$ such that for every $F \subset N$ and $\xi<\omega_{1}$, if $F \in S_{\xi}[N]$ then $F \backslash\{\min F\} \in S_{\xi}^{M}[N]$.

(For a proof see also [AMT].)

Repeated Averages Hierarchy or RA-Hierarchy. We denote by $S_{\ell_{1}}^{+}$the positive part of the unit sphere of $\ell_{1}(\mathbb{N})$, i.e. the set of all sequences $\left(a_{n}\right)_{n=1}^{\infty}$ of non-negative numbers such that $\sum_{n=1}^{\infty} a_{n}=1$. We let $\left(e_{n}\right)_{n=1}^{\infty}$ be the usual basis of $\ell_{1}(\mathbb{N})$. For every countable ordinal $\xi$ and every infinite subset $M$ of $\mathbb{N}$ there exists a sequence $\left(\xi_{n}^{M}\right)_{n \in \mathbb{N}}$ satisfying the following properties:

(1) For every $n \in \mathbb{N}, \xi_{n}^{M} \in S_{\ell_{1}}^{+}$if $\xi>0$, and $\xi_{n}^{M}(m) \leq \frac{1}{\min M}$ for all $m \in M$.

(2) $\operatorname{supp}_{n}^{M}<\operatorname{supp} \xi_{n+1}^{M}$ and $\operatorname{supp} \xi_{n}^{M} \in S_{\xi}$.

(3) $M=\bigcup_{n=1}^{\infty} \operatorname{supp} \xi_{n}^{M}$.

(4) If $\left(n_{k}\right)_{k=1}^{\infty}$ is a strictly increasing sequence, then setting $M^{\prime}=\bigcup_{k=1}^{\infty} \operatorname{supp} \xi_{n_{k}}^{M}$ we have that $\xi_{k}^{M^{\prime}}=\xi_{n_{k}}^{M}$ for all $k \in \mathbb{N}$. 
We recall the definition of $\left(\xi_{n}^{M}\right)_{n \in \mathbb{N}}$ from [AMT]. It is given by transfinite induction.

(1) For $\xi=0, M=\left\{m_{1}, m_{2}, \ldots\right\}, m_{1}<m_{2}<\ldots$ we set $\xi_{n}^{M}=e_{m_{n}}$.

(2) For $\xi=\zeta+1$ assume that for all $M \in[\mathbb{N}]$ the sequence $\left(\zeta_{n}^{M}\right)_{n \in \mathbb{N}}$ has been defined. We set $M=\left(m_{n}\right)_{n=1}^{\infty}$ and $\xi_{1}^{M}=\frac{\zeta_{1}^{M}+\ldots+\zeta_{m_{1}}^{M}}{m_{1}}$. Then we inductively define $\xi_{n}^{M}=\xi_{1}^{M_{n}}$, where $M_{1}=M, M_{n}=M \backslash \bigcup_{i=1}^{n-1} \operatorname{supp} \xi_{i}^{M}$.

(3) If $\xi$ is a limit ordinal we consider the sequence $\left(\xi_{n}\right)_{n \in \mathbb{N}}$ of successor ordinals used in the definition of $S_{\xi}$. For any $M=\left\{m_{1}, m_{2}, \ldots\right\}, M \in[\mathbb{N}]$, we set $n_{1}=$ $m_{1}, \xi_{1}^{M}=\left(\xi_{n_{1}}\right)_{1}^{M}$ and we inductively define $M_{k}=M_{k-1} \backslash \operatorname{supp}\left[\xi_{n_{k-1}}\right]_{1}^{M_{k-1}}$, $n_{k}=\min M_{k}, \xi_{k}^{M}=\left(\xi_{n_{k}}\right)_{1}^{M_{k}}$. By standard induction one could verify properties (1)-(4) listed above.

The following dichotomy principle is important for our proofs; it permits us to stabilize functions acting on the summability methods $\left(\xi_{n}^{M}\right)_{n \in \mathbb{N}}$ without losing the order $\xi$. It is a consequence of stability property (4) of the repeated averages hierarchy and the following well known infinite Ramsey's Theorem.

Theorem 5.2 (Nash-Williams, Galvin and Prikry, Silver, Ellentuck). Let $A$ be an analytic subset of $[\mathbb{N}]$. Then for every $M \in[\mathbb{N}]$ there exists $L \in[M]$ such that either $[L] \subset A$ or $[L] \subset[\mathbb{N}] \backslash A$.

(For a proof we refer to $\mathrm{Ke}$ or $[\mathrm{T}$. .)

Proposition 5.3 (Dichotomy Principle). Let $\xi<\omega_{1}, M \in[\mathbb{N}]$ and $n \in \mathbb{N}$. We set $I_{M, n}=\left\{\left(\xi_{k_{1}}^{N}, \ldots, \xi_{k_{n}}^{N}\right): k_{1}<\ldots<k_{n}, N \in[M]\right\}$. Clearly $I_{M, n} \subset\left(S_{\ell_{1}}^{+}\right)^{n}$. Then for every real function $\phi: I_{M, n} \rightarrow \mathbb{R}$ and $\tau \in \mathbb{R}$ one of the following holds:

(i) There exists $L \in[M]$ such that $\phi(s)>\tau$ for all $s \in I_{L, n}$.

(ii) There exists $L \in[M]$ such that $\phi(s) \leq \tau$ for all $s \in I_{L, n}$.

Definition 5.2. An adequate family $\mathfrak{F}$ of subsets of $\mathbb{N}$ is said to be

(a) $(\xi, M, \varepsilon)$-large, where $\xi<\omega_{1}, M \in[\mathbb{N}]$ and $\varepsilon>0$, if for every $L \in[M]$ and $n \in \mathbb{N}$ there exists $F \in \mathfrak{F}$ such that $\left\langle\xi_{n}^{L}, F\right\rangle=\sum_{k \in F} \xi_{n}^{L}(k)>\varepsilon$;

(b) $(n, \zeta, M, \varepsilon)$-large if for every $N \in[M], \sup _{F \in \mathcal{F}} \inf _{i}\left\{\left\langle\zeta_{i}^{N}, F\right\rangle\right\}_{i=1}^{n}>\varepsilon$.

The following result has been proved in [AMT] and it will be one of the basic tools for our proofs.

Theorem 5.4. Let $\mathfrak{F}$ be an adequate family of finite subsets of $\mathbb{N}$ which is $(\xi, M, \varepsilon)$ large $((n, \xi, M, \varepsilon)$-large for some $n \in \mathbb{N})$ for some countable ordinal $\xi, M \in[\mathbb{N}]$ and $\varepsilon>0$. Then there exists an infinite subset $L$ of $M$ such that $S_{\xi}^{L} \subset \mathfrak{F}\left(\left[S_{\xi}^{L}\right]^{n} \subset \mathfrak{F}\right)$.

Proof. For $n=1$ the result follows from Proposition 2.32 and Theorem 2.26 of AMT. If $n \geq 2$ the result follows from Lemma 2.3.5 in AMT and the Remark after Corollary 2.2.8 in AMT.

From Lemma 5.1 and Theorem 5.4 we obtain the following.

Corollary 5.5. Let $\mathfrak{F}$ be a $(\xi, M, \varepsilon)$-large family. For any $\delta>0$ there exists $L \in$ $[M]$ such that for every $N \in[L]$ and $n \in \mathbb{N}$ there exists $F \in \mathfrak{F}$ with the property $\left\langle\xi_{n}^{N}, F\right\rangle>1-\delta$. 
For a more detailed study of Schreier families and RA-Hierarchy we refer to AG.

Notation. Let $X$ be a Banach space with a basis. We denote by:

(1) $\Sigma(X)$ the set of all normalized block sequences of the basis, i.e. $\Sigma(X)=$ $\left\{\left(x_{i}\right)_{i=1}^{\infty}: x_{i}<x_{i+1},\left\|x_{i}\right\|=1\right.$ and $\left.x_{i} \in X\right\}$.

(2) $\Sigma(\mathbb{N})=\left\{\left(F_{i}\right)_{i=1}^{\infty}: F_{i}<F_{i+1}, F_{i} \subset \mathbb{N}\right\}$.

(3) For every $\mathcal{F}=\left(F_{i}\right)_{i=1}^{\infty} \in \Sigma(\mathbb{N})$ and $s=\left(y_{i}\right)_{i=1}^{\infty} \in \Sigma(X)$ we set $Y_{F_{i}}=$ $\left\langle\left(y_{j}\right)_{j \in F_{i}}\right\rangle$ and

$$
\Sigma(s, \mathcal{F})=\left\{\left(z_{i}\right)_{i=1}^{\infty}: z_{i} \in Y_{i}(\mathcal{F}, s),\left\|z_{i}\right\|=1\right\}
$$

(4) For $s=\left(x_{i}\right)_{i \in \mathbb{N}} \in \Sigma(X)$ we set $\xi_{n}^{M} \cdot s=\sum_{i=1}^{\infty} \xi_{n}^{M}(i) x_{i}$.

(5) If $\xi$ is a countable ordinal, $M \in[\mathbb{N}]$ and $n \in \mathbb{N}$ we set $\left\|\xi_{n}^{M}(s, \mathcal{F})\right\|=$ $\max \left\{\left\|\xi_{n}^{M} \cdot t\right\|, t \in \Sigma(s, \mathcal{F})\right\}$.

(6) Similarly $\left\|\frac{\xi_{1}^{N}+\ldots+\xi_{n}^{N}}{n}(s, \mathcal{F})\right\|=\max \left\{\left\|\frac{\xi_{1}^{N} \cdot t+\ldots+\xi_{n}^{N} \cdot t}{n}\right\|, t \in \Sigma(s, \mathcal{F})\right\}$.

(7) For $s_{1}, s_{2} \in \Sigma(X)$ we write $s_{2} \prec s_{1}$ if $s_{2}$ is a normalized block sequence of $s_{1}$.

(8) If $s_{1}=\left(x_{i}\right)_{i=1}^{\infty}$ and $s_{2}=\left(x_{i}\right)_{i=n}^{\infty}$, for some $n \in \mathbb{N}$, we say that $s_{2}$ is a tail subsequence of $s_{1}$.

(9) If $Y_{1}=\overline{\operatorname{span}}\left[\left(x_{i}\right)_{i=1}^{\infty}\right]$ and $Y_{2}=\overline{\operatorname{span}}\left[\left(x_{i}\right)_{i=n}^{\infty}\right]$ we say that $Y_{2}$ is a tail subspace of $Y_{1}$.

Definition 5.3. Let $X$ be a Banach space with a basis. For every $s \in \Sigma(X)$, $\xi<\omega_{1}$, we set

$$
\begin{aligned}
\tau_{\xi}(s)= & \sup _{\mathcal{F} \in \Sigma(\mathbf{N})} \sup _{M \in[\mathbf{N}]} \inf _{N \in[M]}\left\|\xi_{1}^{N}(s, \mathcal{F})\right\| \text { and } \\
& \tau_{\xi}(X)=\inf _{s \in \Sigma(X)} \tau_{\xi}(s) .
\end{aligned}
$$

This is a complicated definition and we will attempt to explain its necessity after Lemma 5.6.

Remark 5.1. We observe that

1. $\inf _{N \in[M]}\left\|\xi_{1}^{N}(s, \mathcal{F})\right\|=\inf _{N \in[M]} \inf _{n \in \mathbf{N}}\left\|\xi_{n}^{N}(s, \mathcal{F})\right\|$. This follows from stability property (4) of $\left(\xi_{n}^{N}\right)_{n \in \mathbb{N}}$.

2. For $\xi=0$ we have $\tau_{\xi}(X)=1$. For every $0<\xi<\omega_{1}$ and $s \in \Sigma(X)$ we have that $0 \leq \tau_{\xi}(s) \leq 1$.

Lemma 5.6. Suppose that for some $s \in \Sigma(X), \xi<\omega_{1}$ we have that $\tau_{\xi}(s)=\delta>0$. Then the following properties hold:

(1) For every $\mathcal{F}=\left(F_{i}\right)_{i=1}^{\infty} \in \Sigma(\mathbb{N})$ and $M \in[\mathbb{N}]$ there exists $L \in[M]$ such that $\left\|\xi_{n}^{N}(s, \mathcal{F})\right\|<2 \delta$ for every $N \in[L]$ and $n \in \mathbb{N}$.

(2) There exist $\mathcal{F}=\left(F_{i}\right)_{i=1}^{\infty} \in \Sigma(\mathbb{N})$ and $M \in[\mathbb{N}]$ such that for all $N \in[M]$, we have that $\left\|\xi_{1}^{N}(s, \mathcal{F})\right\|>\frac{1}{2} \delta$.

(3) There exist $\mathcal{F} \in \Sigma(\mathbb{N})$ and $M \in[\mathbb{N}]$ such that for all $N \in[M]$ we have that $\frac{1}{2} \delta<\left\|\xi_{1}^{N}(s, \mathcal{F})\right\|<2 \delta$.

Proof. Property (1) follows from Proposition 5.3 (the Dichotomy Principle) and property (2) follows directly from the definition. Property (3) is a combination of (1) and (2). 
To explain the nature of the quantity $\tau_{\xi}(s)$ let us assume that $\tau_{\xi}(s)=\delta>0$. Then from the previous Lemma [5.6 (2) we have that there exist $\mathcal{F} \in \Sigma(\mathbb{N})$ and $M \in[\mathbb{N}]$ such that $\left\|\xi_{1}^{N}(s, \mathcal{F})\right\|>\frac{\delta}{2}$ for every $N \in[M]$. It follows readily that for every $N \in[M]$ there exists $t^{N} \in \Sigma(s, \mathcal{F})$ such that $\left\|\xi_{1}^{N} \cdot t\right\|>\frac{\delta}{2}$. The difficulty is that by changing from $N$ to $L$ it is possible that the sequence $t^{N}$ will also change to some other sequence $t^{L}$. This forces us to be more careful in our proofs. The advantage of the above definition comes essentially from part (1) of Lemma 5.6. Indeed, if for a given $\varepsilon>0$ we knew that $0<\tau_{\xi}(s)=\delta<\varepsilon$, then for every $\mathcal{F} \in \Sigma(\mathbb{N})$ and every $M \in[\mathbb{N}]$ there exists $L \in[M]$ such that $\left\|\xi_{1}^{N}(s, \mathcal{F})\right\|<2 \delta<2 \varepsilon$ for all $N \in[L]$. This permits us to have a uniform $2 \delta$-control over all convex combinations of a certain form and at the same time we know by part two of Lemma 5.6 that there are convex combinations of the same form which remain greater than $\frac{\delta}{2}$. This key observation is crucial for our approach.

Lemma 5.7. Let $X$ be a Banach space with a basis and $\xi<\omega_{1}$.

(a) If $s_{1}, s_{2} \in \Sigma(X)$ and $s_{2} \prec s_{1}$, then $\tau_{\xi}\left(s_{1}\right) \geq \tau_{\xi}\left(s_{2}\right)$.

(b) If $Y \prec Z \prec X$, then $\tau_{\xi}(Y) \geq \tau_{\xi}(Z)$.

(c) If $s_{1}, s_{2} \in \Sigma(X)$ and $s_{2}$ is a tail subsequence of $s_{1}$, then $\tau_{\xi}\left(s_{1}\right)=\tau_{\xi}\left(s_{2}\right)$.

(d) If $Z$ is a tail subspace of $Y$, then $\tau_{\xi}(Y)=\tau_{\xi}(Z)$.

Proof. (a) It is enough to show that if for $\theta \in(0,1)$ we have $\tau_{\xi}\left(s_{2}\right)>\theta$, then also $\tau_{\xi}\left(s_{1}\right)>\theta$. Suppose that $\tau_{\xi}\left(s_{2}\right)>\theta$ for some $\theta \in(0,1)$. Then there exist $\mathcal{F}=\left(F_{n}\right)_{n=1}^{\infty} \in \Sigma(\mathbb{N})$ and $M \in[\mathbb{N}]$ such that for every $N \in[M]$ we can select $t^{N} \in$ $\Sigma\left(s_{2}, \mathcal{F}\right)$ with $\left\|\xi_{1}^{N} \cdot t^{N}\right\|>\theta$. Let $s_{1}=\left(y_{n}\right)_{n=1}^{\infty}$ and $s_{2}=\left(z_{n}\right)_{n=1}^{\infty}, z_{n}=\sum_{i \in E_{n}} \lambda_{i} y_{i}$, where $\left(E_{n}\right)_{n=1}^{\infty}$ is a sequence of successive subsets of $\mathbb{N}$.

We set $F_{n}^{\prime}=\bigcup_{i \in F_{n}} E_{i}, \mathcal{F}^{\prime}=\left(F_{n}^{\prime}\right)_{n=1}^{\infty}$. Then $\Sigma\left(s_{2}, \mathcal{F}^{\prime}\right) \subset \Sigma\left(s_{1}, \mathcal{F}^{\prime}\right)$ and therefore for every $N \in[M]$ the sequence $t^{N}$ also belongs to $\Sigma\left(s_{1}, \mathcal{F}^{\prime}\right)$. We thus obtain that $\left\|\xi_{1}^{N} \cdot t^{N}\right\|>\theta$.

Properties (b), (c), (d) are obvious.

Lemma 5.8. For every $\xi<\omega_{1}$ there exists a block subspace $Y$ of $X$ such that $\tau_{\xi}(s)=\tau_{\xi}(Y)$ for every $s \in \Sigma(Y)$.

Proof. We inductively define sequences $\left(s_{i}\right)_{i=1}^{\infty},\left(Y_{i}\right)_{i=1}^{\infty}$ such that $s_{1}=\left(e_{n}\right)_{n \in \mathbb{N}}$ is the basis of $X, s_{n+1} \prec s_{n}, Y_{i}=\overline{\operatorname{span}}\left[s_{i}\right], s_{i+1} \in \Sigma\left(Y_{i}\right)$ and $\tau_{\xi}\left(s_{i+1}\right)-\tau_{\xi}\left(Y_{i}\right)<\frac{1}{i+1}$. Let $s$ be a diagonal block sequence of $\left(s_{i}\right)_{i=1}^{\infty}$ and set $Y=\overline{\operatorname{span}}[s]$. If $t \in \Sigma(Y)$, then for every $k$ there exists $t_{k} \prec s_{k}$, a tail subsequence of $t$, which satisfies $\tau_{\xi}\left(Y_{k}\right) \leq$ $\tau_{\xi}\left(t_{k}\right)=\tau_{\xi}(t)=\tau_{\xi}\left(t_{k+1}\right) \leq \tau_{\xi}\left(s_{k+1}\right)$, by Lemma 5.7 (a) and (c). Therefore for $t, t^{\prime} \in \Sigma(Y),\left|\tau_{\xi}(t)-\tau_{\xi}\left(t^{\prime}\right)\right|<\frac{1}{k}$ for all $k \in \mathbb{N}$ and hence $\tau_{\xi}(t)=\tau_{\xi}\left(t^{\prime}\right)$.

The following definition is the natural extension of Definition 5.3 to $n$-averages of $\left(\xi_{k}^{N}\right)_{k \in \mathbb{N}}$ and it will be used at a certain stage of our proof.

Definition 5.4. (a) For every $s \in \Sigma(X), \xi<\omega_{1}, n \in \mathbb{N}$ we set:

$$
\tau_{\xi, n}(s)=\sup _{\mathcal{F} \in \Sigma(\mathbf{N})} \sup _{M \in[\mathbf{N}]} \inf _{N \in[M]}\left\|\frac{\xi_{1}^{N}+\ldots+\xi_{n}^{N}}{n}(s, \mathcal{F})\right\| .
$$

(b) We define $\tau_{\xi, n}(X)=\inf _{s \in \Sigma(X)} \tau_{\xi, n}(s)$. 
Remark 5.2. (a) For $n=1$ the above definition coincides with Definition 5.3.

(b) The corresponding results of Lemmas [5.7] and [5.8 stated for $\tau_{\xi, n}$ instead of $\tau_{\xi}$ also hold and the proofs are completely analogous. In particular for a given $\xi<\omega_{1}$, $n \in \mathbb{N}$, there exists a block subspace $Y$ of $X$ such that $\tau_{\xi, n}(Y)=\tau_{\xi, n}(s)$ for every $s \in \Sigma(Y)$.

Definition 5.5. For $\delta>0, s=\left(y_{i}\right)_{i=1}^{\infty} \in \Sigma(X)$ and $\mathcal{F}=\left(F_{i}\right)_{i=1}^{\infty} \in \Sigma(\mathbb{N})$ we define: $\mathfrak{F}_{\delta}(s, \mathcal{F})=\left\{G \in[\mathbb{N}]^{<\infty}: \exists x_{G}^{*} \in S_{X^{*}}\right.$ such that $\left.\left\|\left.x_{G}^{*}\right|_{Y_{F_{l}}}\right\|>\delta \quad \forall l \in G\right\}$, where $Y_{F_{l}}=\operatorname{span}\left[\left(y_{i}\right)_{i \in F_{l}}\right]$.

Remark 5.3. If $X$ is a reflexive Banach space with a 1-unconditional basis, then it is easy to check that the set $\mathfrak{F}_{\delta}(s, \mathcal{F})$ is an adequate and compact family of finite subsets of $\mathbb{N}$.

In the sequel $X$ will denote a reflexive Banach space with a 1-unconditional basis.

Lemma 5.9. Suppose that for some $\xi<\omega_{1}, s=\left(y_{i}\right)_{i=1}^{\infty} \in \Sigma(X)$ and $\mathcal{F}=$ $\left(F_{i}\right)_{i=1}^{\infty} \in \Sigma(\mathbb{N})$ there exists $M \in[\mathbb{N}]$ and $\delta>0$ such that $\left\|\xi_{1}^{N}(s, \mathcal{F})\right\|>\delta$ for all $N \in[M]$. Then there exists $L \in[M]$ such that $S_{\xi}^{L} \subset \mathfrak{F}_{\delta / 2}(s, \mathcal{F})$, i.e. for every $G \in S_{\xi}^{L}$ there exists $x_{G}^{*} \in S_{X^{*}}$ satisfying $\left\|\left.x_{G}^{*}\right|_{Y_{F_{l}}}\right\|>\frac{\delta}{2}$ for every $l \in G$.

Proof. It follows from stability property (4) of the summability methods that for all $n \in \mathbb{N}, N \in[M]$ we have that $\left\|\xi_{n}^{N}(s, \mathcal{F})\right\|>\delta$. Further, given $t \in \Sigma(s, \mathcal{F})$ such that $\left\|\xi_{n}^{N} \cdot t\right\|>\delta$ we choose $x^{*} \in S_{X^{*}}$ such that $x^{*}\left(\xi_{n}^{N} \cdot t\right)>\delta$. It follows that $\sum\left\{\xi_{n}^{N}(l):\left\|\left.x^{*}\right|_{Y_{F_{l}}}\right\|>\frac{\delta}{2}\right\}>\frac{\delta}{2}$. Therefore the compact and adequate family $\mathfrak{F}_{\delta / 2}(\mathbf{s}, \mathcal{F})$ is $\left(\xi, M, \frac{\delta}{2}\right)$-large.

The result follows from Theorem 5.4 and the proof is complete.

Lemma 5.10. Let $s \in \Sigma(X), \mathcal{F} \in \Sigma(\mathbb{N}), M \in[\mathbb{N}]$ and $\delta>0$. Assume that for $k>n\left(\frac{2}{\delta}+1\right)$ and $\zeta<\omega_{1}$ we have that for all $N \in[M],\left\|\frac{\zeta_{1}^{N}+\ldots+\zeta_{l}^{N}}{k}(s, \mathcal{F})\right\|>\delta$. Then there exists $L \in[M]$ such that $\left[S_{\zeta}^{L}\right]^{n} \subset \mathfrak{F}_{\delta / 4}(s, \mathcal{F})$.

Proof. We observe that if $x^{*} \in B_{X^{*}}, t \in \Sigma(s, \mathcal{F})$ are such that $x^{*}\left(\frac{\zeta_{1}^{N}+\ldots+\zeta_{k}^{N}}{k} \cdot t\right)>\delta$, then there exist $\left\{k_{1}<k_{2}<\ldots<k_{n}\right\} \subset\{1,2, \ldots, k\}$ such that $x^{*}\left(\zeta_{k_{i}}^{N} \cdot t\right)>\frac{\delta}{2}$ for all $i=1, \ldots, n$. Hence we can define a partition of $[M]=\bigcup\left\{A_{\left(k_{1}, \ldots, k_{n}\right)}: 1 \leq k_{1}<\ldots<\right.$ $\left.k_{n} \leq k\right\}$ with the rule $N \in A_{\left(k_{1}, \ldots, k_{n}\right)}$ if and only if there exists $x^{*} \in B_{X^{*}}$ with $x^{*}\left(\zeta_{k_{i}}^{N} \cdot t\right)>\frac{\delta}{2}$ for all $i=1, \ldots, n$.

From the dichotomy principle (Proposition [5.3) there exist $L \in[M]$ and $1 \leq$ $k_{1}<\ldots<k_{n} \leq k$ such that $[L] \subset A_{\left(k_{1}, \ldots, k_{n}\right)}$. It follows from stability property (4) of the repeated averages hierarchy that for every $N \in[L]$ there exists $N^{\prime} \subset N$ such that $N^{\prime} \subset A_{(1, \ldots, n)}$. From this we can conclude that actually $[L] \subset A_{(1, \ldots, n)}$. Let $N \in[L]$ and choose $x^{*} \in B_{X^{*}}$ such that $x^{*}\left(\zeta_{i}^{N} \cdot t\right)>\frac{\delta}{2}$ for $i=1, \ldots, n$. Then for every $i=1, \ldots, n$ we get that $\sum\left\{\zeta_{i}^{N}(d):\left\|x^{*} \mid Y_{F_{d}}\right\|>\frac{\delta}{4}\right\}>\frac{\delta}{4}$. Therefore $\mathfrak{F}_{\delta / 4}(s, \mathcal{F})$ is $\left(n, \zeta, L, \frac{\delta}{4}\right)$-large and the result follows from Theorem 5.4

Lemma 5.11. Let $\zeta<\omega_{1}, s \in \Sigma(X), \mathcal{F}=\left(F_{n}\right)_{n \in \mathbb{N}} \in \Sigma(\mathbb{N}), \delta>0$ and $k \in \mathbb{N}$. Assume that for some $L \in[\mathbb{N}]$ we have that $\left[S_{\zeta}^{L}\right]^{k} \subset \mathfrak{F}_{\delta}(s, \mathcal{F})$. Then there exists $\mathcal{G} \in \Sigma(\mathbb{N}), \mathcal{G} \subset \mathcal{F}$, such that for every $\mathcal{G}^{\prime} \subset \mathcal{G}$ and $M \in[\mathbb{N}]$ we have that $\left[S_{\zeta}\right]^{k} \subset$ $\mathfrak{F}_{\delta}\left(s, \mathcal{G}^{\prime}\right)$ and $\left\|\frac{\zeta_{1}^{M}+\ldots+\zeta_{k}^{M}}{k}\left(s, \mathcal{G}^{\prime}\right)\right\|>\delta$. 
Proof. Let $L=\left(l_{i}\right)_{i \in \mathbb{N}}$. We set $\mathcal{G}=\left\{G_{i}\right\}_{i \in \mathbb{N}}=\left\{F_{l_{i}}\right\}_{i \in \mathbb{N}}$. It follows from the definition of $S_{\xi}^{L}$ and the choice of the family $\mathcal{G}$ that $\left[S_{\xi}\right]^{k} \subset \mathfrak{F}_{\delta}(s, \mathcal{G})$. If $\mathcal{G}^{\prime} \subset \mathcal{G}$ and $\mathcal{G}^{\prime} \subset \Sigma(\mathbb{N})$, then there exists $M \in[L]$ such that $\mathcal{G}^{\prime}=\left(F_{l_{i}}\right)_{i \in M}$. Set $M^{\prime}=\left(l_{i}\right)_{i \in M}$. Then $M^{\prime} \in[L]$ and it is easy to check that $\left[S_{\zeta}^{M^{\prime}}\right]^{k} \subset\left[S_{\zeta}^{L}\right]^{k} \subset \mathfrak{F}_{\delta}(s, \mathcal{G})$. If $M^{\prime}=$ $\left(m_{i}\right)_{i \in \mathbb{N}}$, then $\mathcal{G}^{\prime}=\left(F_{m_{i}}\right)_{i \in \mathbb{N}}$ and as in the case of $\mathcal{G}$ we get that $\left[S_{\zeta}\right]^{k} \subset \mathfrak{F}_{\delta}\left(s, \mathcal{G}^{\prime}\right)$. Therefore for every $M \in[\mathbb{N}],\left\|\frac{\zeta_{1}^{M}+\ldots+\zeta_{k}^{M}}{k}\left(s, \mathcal{G}^{\prime}\right)\right\|>\delta$ and the proof is complete.

Remark 5.4. The content of the above lemmas is that at the moment when we know that $\left\|\frac{\zeta_{1}^{N}+\ldots+\zeta_{k}^{N}}{k}(s, \mathcal{F})\right\|>\delta$ for some $\delta>0$, the pair $(s, \mathcal{F})$ and all $N \in[L]$, then by passing to a certain $\mathcal{G} \subset \mathcal{F}$ a similar inequality holds for the pair $(s, \mathcal{G})$, the number $\frac{\delta}{4}$ instead of $\delta$, and, what is most important, for all $L$ subsets of $\mathbb{N}$.

Lemma 5.12. Let $s \in \Sigma(X)$ and $\xi<\omega_{1}$ such that $\tau_{\xi}(s)>\delta>0$. Then for every $\zeta<\xi, \tau_{\zeta}(s)>\frac{\delta}{2}$.

Proof. Lemmas 5.9 and 5.11 (see also Remark 5.4) yield $\mathcal{G} \subset \mathcal{F}$ such that $S_{\xi} \subset$ $\mathfrak{F}_{\frac{\delta}{2}}(s, \mathcal{G})$. By property $(5)$ of the Schreier families there exists $n \in \mathbb{N}$ such that $S_{\zeta}[\mathbb{N} \backslash\{1, \ldots, n-1\}] \subset S_{\xi} \subset \mathfrak{F}_{\frac{\delta}{2}}(s, \mathcal{G})$. Then for $L \in[\mathbb{N} \backslash\{1, \ldots, n-1\}]$ it follows that $\zeta_{1}^{L}(s, \mathcal{G})>\frac{\delta}{2}$ and hence $\tau_{\zeta}(s)>\frac{\delta}{2}$.

Remark 5.5. It follows from Lemma 5.12 that if $\tau_{\xi}(s)=0$ for some $\xi<\omega_{1}$ and $s \in \Sigma(X)$, then $\tau_{\xi^{\prime}}(s)=0$ for every $\xi^{\prime} \geq \xi$.

Before passing to the next result we make a brief introduction to $c_{0}$-trees. A tree $\mathcal{T}$ is said to be well-founded if every linearly ordered subset of $\mathcal{T}$ is finite. For a well-founded tree we define the derivative tree $\mathcal{T}^{\prime}$ to be the subtree of $\mathcal{T}$ consisting of all non-maximal elements of $\mathcal{T}$; then we inductively define the $\xi$-derivative as $\mathcal{T}^{(\zeta+1)}=\left(\mathcal{T}^{(\zeta)}\right)^{\prime}$ and $\mathcal{T}^{(\xi)}=\bigcap_{\zeta<\xi} \mathcal{T}^{(\zeta)}$ if $\xi$ is a limit ordinal. The order $\mathbf{o}(\mathcal{T})$ of a well-founded tree $\mathcal{T}$ is the smallest ordinal $\xi$ such that the $\xi$-derivative of $\mathcal{T}$ is an empty set.

$c_{0}$-trees: Let $X$ be a separable Banach space. For $\delta>0$, we define $\mathcal{T}\left(c_{0}, \delta\right)=$ $\left\{\left(x_{1}, \ldots, x_{n}\right):\left\|x_{i}\right\|=1\right.$ and $\left(x_{1}, \ldots, x_{n}\right)$ is $\frac{1}{\delta}$-equivalent to the usual basis of $\left.\ell_{n}^{\infty}\right\}$. Then $\mathcal{T}\left(c_{0}, \delta\right)$ with the natural order is a tree and further if $c_{0}$ is not isomorphic to a subspace of $X$, then $\mathcal{T}\left(c_{0}, \delta\right)$ is a well-founded tree. The $c_{0}$-index of a space $X$ not containing $c_{0}$ is defined as $\mathbf{o}(X)=\sup \left\{\mathbf{o}\left(\mathcal{T}\left(c_{0}, \delta\right)\right): \delta>0\right\}$.

The following result is due to J. Bourgain [Bo1]:

Theorem 5.13. Let $X$ be a separable Banach space not containing $c_{0}$. Then the $c_{0}$-index $\mathbf{o}(X)$ is a countable ordinal.

In the next proposition we will show that the assumption $\tau_{\xi}(s)>0$ implies that $\mathbf{o}\left(X^{*}\right) \geq \xi$ and from this we will conclude that there exists a countable ordinal $\zeta_{0}$ such that $\tau_{\xi}(s)=0$ for all $s \in \Sigma(X)$ and $\xi \geq \zeta_{0}$. We begin with some notation and preliminary remarks.

(a) For $s=\left(y_{l}\right)_{l \in \mathbb{N}} \in \Sigma(X), \mathcal{F}=\left(F_{k}\right) \in \Sigma(\mathbb{N})$ and $k \in \mathbb{N}$ we denote $\tilde{Y}_{F_{k}}=$ $\operatorname{span}\left[e_{i}: e_{i} \in \bigcup_{l \in F_{m_{k}}}\right.$ suppy $\left._{l}\right]$ and we denote by $\operatorname{supp} \tilde{Y}_{F_{k}}$ the set $\left\{i: e_{i} \in \tilde{Y}_{F_{k}}\right\}$.

(b) For $s \in \Sigma(X), \mathcal{F} \in \Sigma(\mathbb{N})$ and $\delta>0$ consider $\mathfrak{D} \subset \mathfrak{F}_{\delta}(s, \mathcal{F})$. We denote by $\mathcal{C}(\mathfrak{D})$ any set $\left\{x_{G}^{*}: G \in \mathfrak{D}\right\} \subset B_{X^{*}}$ where each $x_{G}^{*}$ witnesses that $G \in \mathfrak{F}_{\delta}(s, \mathcal{F})$. Observe that from the definition of $\mathfrak{F}_{\delta}(s, \mathcal{F})$ and the fact that $Y_{F_{k}}$ is a subspace 
of $\tilde{Y}_{F_{k}}$ we get that if $G \in \mathfrak{F}_{\delta}(s, \mathcal{F})$ and $G=\left\{m_{1}, \ldots, m_{d}\right\}$, then for all $1 \leq i \leq d$, $\left.x_{G}^{*}\right|_{\tilde{Y}_{F_{m_{i}}}} \in X^{*}$ and $\left\|\left.x_{G}^{*}\right|_{\tilde{Y}_{F_{m_{i}}}}\right\|>\delta$. Therefore if $X$ has a 1-unconditional basis, then $\left\{\left.x_{G}^{*}\right|_{\tilde{Y}_{F_{m_{i}}}}: i=1, \ldots, d\right\}$ is $\frac{1}{\delta}$ equivalent to the uvb of $c_{0}(d)$. Furthermore if $\varepsilon<\frac{\delta}{2}$ and $\left\{x_{i}^{*}\right\}_{i=1}^{d}$ are such that $\sum_{i=1}^{d}\left\|\left.x_{G}^{*}\right|_{\tilde{Y}_{F_{k}}}-x_{i}^{*}\right\|<\varepsilon$, then $\left\{x_{i}^{*}: i=1, \ldots, d\right\}$ is $\frac{1}{\delta^{2}}$ equivalent to the uvb of $c_{0}(d)$.

Lemma 5.14. Assume that for $\mathfrak{D} \subset \mathfrak{F}_{\delta}(s, \mathcal{F})$ and $\mathcal{C}(\mathfrak{D})=\left\{x_{G}^{*}: G \in \mathfrak{D}\right\}$ there exists $M \in[\mathbb{N}]$ such that $S_{\xi}[M] \subset \mathfrak{D}$. Then for every $k_{0} \in \mathbb{N}$ there exists $L \in[M]$ and $x^{*} \in B_{X^{*}}$ such that the following hold:

(i) $\operatorname{suppx}^{*} \subset \operatorname{supp} \tilde{Y}_{F_{m_{k_{0}}}}$.

(ii) $m_{k_{0}}<\min L$ and if $G \subset\left\{m_{k_{0}}\right\} \cup L$ is a maximal member of $S_{\xi}$ with $\min G=m_{k_{0}}$, then $\left\|\left.x_{G}^{*}\right|_{\tilde{Y}_{F_{m_{k}}}}-x^{*}\right\|<\varepsilon$.

Proof. Since $Y_{F_{m_{k}}}^{*}$ is a finite-dimensional subspace of $X^{*}$ there exists an $\varepsilon$-net $\left\{x_{1}^{*}, \ldots, x_{d}^{*}\right\}$ in $B_{\tilde{Y}_{F_{m_{k}}}^{*}}$. We recall that property (4) of Schreier families implies that for $L \in[M], L=\left(l_{j}\right)_{j=1}^{\infty}$ there exists $d \in \mathbb{N}$ so that $\left(l_{j}\right)_{j=1}^{d}$ is a maximal element of $S_{\xi}$. For $1 \leq i \leq d$ define the set

$$
A_{i}=\left\{\begin{array}{c}
N \in\left[M \backslash\left\{m_{i}\right\}_{i=1}^{k_{0}}\right]: \text { if } N^{\prime}=N \cup\left\{m_{k_{0}}\right\} \text { and } G \text { is the unique maximal } \\
\text { element of } S_{\xi} \text { defined by an initial segment of } N^{\prime}, \\
\text { then }\left\|\left.x_{G}^{*}\right|_{\tilde{Y}_{F_{m_{k_{0}}}}-x_{i}^{*} \|}\right\| \varepsilon
\end{array}\right\} .
$$

It is immediate that $\left[M \backslash\left\{m_{i}\right\}_{i=1}^{k_{0}}\right]=\bigcup_{i=1}^{d} A_{i}$ and each $A_{i}$ is open. Hence by the infinite Ramsey Theorem (Theorem [5.2) we obtain $L \in\left[M \backslash\left\{m_{i}\right\}_{i=1}^{k_{0}}\right]$ such that $[L] \subset A_{i_{0}}$ for some $i_{0} \in\{1, \ldots, d\}$. Set $x^{*}=x_{i_{0}}^{*}$ and observe that for every maximal element $G \subset\left\{m_{k_{0}}\right\} \cup L$ of $S_{\xi}$ with $\min G=m_{k_{0}}$ there exists $N \in\left[\left\{m_{k_{0}}\right\} \cup L\right]$ such that $G$ is an initial segment of $N$. Therefore the pair $\left(x^{*}, L\right)$ satisfies the desired conclusion.

Proposition 5.15. Let $X$ be a reflexive Banach space with an unconditional basis. Assume that for some $\xi<\omega_{1}$ and $s \in \Sigma(X), \tau_{\xi}(s)>0$. Then the $c_{0}$-index of $X^{*}$ is greater than or equal to $\xi$.

Proof. We prove by induction the following statement:

The inductive hypothesis: If $\mathfrak{D} \subset \mathfrak{F}_{\delta / 2}(s, \mathcal{F})$ is such that $S_{\xi}[M] \subset \mathfrak{D}$ and $\mathcal{C}(\mathfrak{D})=\left\{x_{G}^{*}: G \in \mathfrak{D}\right\}$, then for every $\varepsilon>0$ there exists a tree $\mathcal{T} \subset\left\{\left(x_{1}^{*}, \ldots, x_{d}^{*}\right)\right.$ : $\left.d \in \mathbb{N}, x_{i}^{*} \in B_{X^{*}}\right\}$ satisfying the following properties:

(i) $\mathbf{o}(\mathcal{T}) \geq \xi$.

(ii) If $\left(x_{1}^{*}, \ldots, x_{d}^{*}\right) \in \mathcal{T}_{\xi}$, then there exists a maximal element $G$ of $S_{\xi}$, contained in $M$ and $G^{\prime} \subset G$ such that $G^{\prime}=\left\{m_{1}, \ldots, m_{d}\right\}, \sum_{i=1}^{d}\left\|\left.x_{G}^{*}\right|_{\tilde{Y}_{F_{m_{i}}}}-x_{i}^{*}\right\|<\varepsilon$ and $\operatorname{supp} x_{i}^{*} \subset \operatorname{supp} \tilde{Y}_{F_{m_{i}}}$.

Proof of the inductive hypothesis: For $\xi=0$ it is obvious. Suppose that $\xi$ is a limit ordinal, $\left(\xi_{n}\right)_{n \in \mathbb{N}}$ is the increasing sequence that defines $S_{\xi}$ and $\mathfrak{D}$, 
$\mathcal{C}(\mathfrak{D}), \varepsilon>0$ are given. Suppose that $M \in[\mathbb{N}]$ such that $S_{\xi}[M] \subset \mathfrak{D}$. We let $M_{n}=\{m \in M: m \geq n\}$. It follows from the definition of $S_{\xi}$ that $S_{\xi_{n}}\left[M_{n}\right] \subset$ $S_{\xi}[M]$. To each $U \in S_{\xi_{n}}\left[M_{n}\right]$ there corresponds a unique maximal element $G_{U}$ of $S_{\xi}[M]$ with $U \subset G_{U}$. Then for the families $\mathfrak{D}_{n}=\left\{U: U \in S_{\xi_{n}}\left[M_{n}\right]\right\}, \mathcal{C}\left(\mathfrak{D}_{n}\right)=$ $\left\{G_{U}: U \in S_{\xi_{n}}\left[M_{n}\right]\right\}$ and the given $\varepsilon>0$ the inductive assumptions are fulfilled and hence there exists $T_{\xi_{n}}$ satisfying conditions $(i)$ and (ii). It is easily checked that the tree $\mathcal{T}_{\xi}=\bigcup_{n} \mathcal{T}_{\xi_{n}}$ satisfies conditions $(i)$ and $(i i)$ for the families $\mathfrak{D}$ and $\mathcal{C}(\mathfrak{D})$ and the given $\varepsilon>0$. This completes the proof for the limit ordinal case.

Suppose now that $\xi=\zeta+1$ and $\mathfrak{D}, \mathcal{C}(\mathfrak{D}), \varepsilon>0$ are given such that $S_{\xi}[M] \subset \mathfrak{D}$. Choose $m_{k_{0}} \in M$ with $m_{k_{0}} \geq 2$. Then by Lemma 5.14 for $\varepsilon^{\prime}=\frac{\varepsilon}{2}$ there exists $x^{*} \in B_{X^{*}}$ with suppx* $\subset \operatorname{supp} \tilde{Y}_{F_{m_{k_{0}}}}$ and $L \in[M]$ such that for every $G \subset L$, $G$ maximal in $S_{\xi}, \min G=m_{k_{0}}$ we have that $\left\|x_{G \mid \tilde{Y}_{F_{m_{k}}}}-x^{*}\right\|<\varepsilon^{\prime}$. Observe that the definition of $S_{\zeta+1}$ implies that for every $G \in S_{\zeta}[L], m_{k_{0}}<G$, the set $\left\{m_{k_{0}}\right\} \cup G \in S_{\xi}$. Therefore we may assign to each $U \in S_{\zeta}[L]$ a $G_{U} \in S_{\xi}\left[L \cup\left\{m_{k_{0}}\right\}\right]$ maximal in $S_{\xi}, U \subset G_{U}, \min G_{U}=m_{k_{0}}$. It is clear that the families $\mathfrak{D}^{\prime}=$ $S_{\zeta}[L], \mathcal{C}\left(\mathfrak{D}^{\prime}\right)=\left\{x_{G_{U}}^{*}: U \in S_{\xi}[L]\right\}$ and the number $\varepsilon^{\prime}=\frac{\varepsilon}{2}$ satisfy the inductive assumptions, hence there exists a tree $\mathcal{T}^{\prime}$ with $\mathbf{o}\left(\mathcal{T}^{\prime}\right) \geq \zeta$ and the elements of $\mathcal{T}^{\prime}$ fulfill property $(i i)$. Define $\mathcal{T}_{\xi}=\left\{\left(x^{*}, x_{1}^{*}, \ldots, x_{d}^{*}\right):\left(x_{1}^{*}, \ldots, x_{d}^{*}\right) \in \mathcal{T}^{\prime}\right\}$. Clearly $\mathcal{T}$ is the desired tree and the inductive hypothesis is completely proved.

To finish the proof we observe first that since $\tau_{\xi}(s)>0$ there exist $\mathcal{F} \in \Sigma(\mathbb{N})$ and $M \in[\mathbb{N}]$ such that, for every $N \in[M],\left\|\xi_{1}^{N}(s, \mathcal{F})\right\|>\frac{\tau_{\xi}(s)}{2}=\delta$. Therefore by Lemma 5.9 there exists $L \in[M]$ such that $S_{\xi}^{L} \subset \mathfrak{F}_{\frac{\delta}{2}}(s, \mathcal{F})$. If $\mathcal{F}=\left(F_{n}\right)_{n \in \mathbb{N}}$ we set $\mathcal{G}=\left(G_{n}\right)_{n \in \mathbb{N}}=\left(F_{k}\right)_{k \in L}$. Then it follows that $S_{\xi} \subset \mathfrak{F}_{\delta / 2}(s, \mathcal{G})$ (Lemma [5.11). To each $G \in \mathfrak{F}_{\delta / 2}(s, \mathcal{G})$ we assign $x_{G}^{*}$ witnessing that $G \in \mathfrak{F}_{\delta / 2}(s, \mathcal{G})$ and we set $\mathcal{C}=\left\{x_{G}^{*}: G \in \mathfrak{F}_{\delta / 2}(s, \mathcal{G})\right\}$. Let $0<\varepsilon<\frac{\delta}{4}$. Then applying the inductive hypothesis for the families $\mathfrak{F}_{\delta / 2}(s, \mathcal{G}), \mathcal{C}$, and the number $\varepsilon$ we get a tree $\mathcal{T}$ which satisfies properties $(i)$ and $(i i)$. Property $(i)$ implies that $\mathbf{o}(\mathcal{T}) \geq \xi$. Also property $(i i)$ and the unconditionality of the basis of $X$ show that every $\left(x_{1}^{*}, \ldots, x_{d}^{*}\right) \in \mathcal{T}$ is $\frac{2}{\delta^{2}}$ equivalent to the uvb of $c_{0}(d)$. Hence indeed $\mathbf{o}\left(X^{*}\right) \geq \xi$.

Proposition 5.16. Every reflexive Banach space $X$ with an unconditional basis contains a subspace $Y$ with the following property: There exists a unique $\xi_{Y}<\omega_{1}$ such that

(1) $\tau_{\xi_{Y}}(Y)=0$ and $\tau_{\xi}(Y) \neq 0$ for every $\xi<\xi_{Y}$, and

(2) for every $\xi<\omega_{1}, s \in \Sigma(Y)$ and $n \in \mathbb{N}, \tau_{\xi, n}(s)=\tau_{\xi, n}(Y)$.

Proof. As was shown in Lemma 5.8, for a countable ordinal $\xi$ and $Z \prec X$ there exists $Y_{\xi} \prec Z$ such that $\tau_{\xi}(s)=\tau_{\xi}\left(Y_{\xi}\right)$. By similar arguments it is also shown that there exists $Y_{\xi, n}$ such that the same holds for the quantity $\tau_{\xi, n}$ (Remark 5.1). For such a subspace we say that $\tau_{\xi, n}$ is stabilized in $Y_{\xi, n}$. Therefore for every $Z \prec X$ and $\xi<\omega_{1}$ we inductively choose $Z \succ Y_{\xi, 1} \succ Y_{\xi, 2} \succ \ldots \succ Y_{\xi, n} \succ \ldots$ such that $\tau_{\xi, n}$ is stabilized in $Y_{\xi, n}$. If $Y_{\xi}$ is any diagonal subspace of $\left\{Y_{\xi, n}\right\}_{n \in \mathbb{N}}$, then $\left(\tau_{\xi, n}\right)_{n \in \mathbb{N}}$ are simultaneously stabilized on $Y$. From Theorem 5.13 and Proposition 5.15 we obtain a countable ordinal $\zeta_{0}$ such that for every $\xi \geq \zeta_{0}$ and $s \in \Sigma(X)$ we have that $\tau_{\xi}(s)=0$. We define $\xi_{X}=\min \left\{\xi\right.$ : for every $\left.s \in \Sigma(X), \tau_{\xi}(s)=0\right\}$. Observe that whenever $\tau_{\xi}(s)=0$, then also $\tau_{\xi, n}(s)=0$ for all $n \in \mathbb{N}$. Clearly $\xi_{X}$ is a countable ordinal and $\xi_{X}>0$. Enumerate the set $\left\{\xi: \xi<\xi_{X}\right\}$ as a sequence 
$\left(\xi_{n}\right)_{n \in \mathbb{N}}$ and inductively choose a sequence $\left(Y_{n}\right)_{n \in \mathbb{N}}$ of block subspaces of $X$ such that $Y_{n+1} \prec Y_{n}$ and $\left(\tau_{\xi_{n}, k}\right)_{k \in \mathbb{N}}$ is stabilized in $Y_{n}$. Let $Y$ be any diagonal subspace of the sequence $\left(Y_{n}\right)_{n \in \mathbb{N}}$. It is readily checked that $Y$ is the desired subspace and $\xi_{Y}$, which is smaller than or equal to $\xi_{X}$, satisfies the desired property (1).

Definition 5.6. A Banach space $Y$ which satisfies the conclusion of the previous proposition is called a stabilized space.

Notation. For $\mathcal{F}_{1}=\left(F_{l}^{1}\right)$ and $\mathcal{F}_{2}=\left(F_{l}^{2}\right)$, we write $\mathcal{F}_{2} \prec \mathcal{F}_{1}$ if there exists $\left(G_{l}\right)_{l \in \mathbb{N}} \in$ $\Sigma(\mathbb{N})$ such that $F_{l}^{2}=\bigcup_{d \in G_{l}} F_{d}^{1}$, for all $l \in \mathbb{N}$.

Proposition 5.17. Let $X$ be a stabilized reflexive space with an unconditional basis. Then the following hold:

(a) If $\xi=\xi_{X}$ is a limit ordinal and $\left(\xi_{n}\right)_{n \in \mathbb{N}}$ is the increasing sequence that defines $S_{\xi}$, then $\lim \tau_{\xi_{n}}=0$.

(b) If $\xi=\xi_{X}$ is of the form $\xi=\zeta+1$, then $\lim _{n} \tau_{\zeta, n}=0$.

Proof. We first show that (a) holds. By Lemma 5.12 it is enough to prove that $\underline{\lim } \tau_{\xi_{n}}=0$. Assume on the contrary that $\underline{\lim } \tau_{\xi_{n}}>0$. Since the space $X$ is stabilized there exists $\delta>0$ such that $\tau_{\xi_{n}}(s)>\delta$ for all $n \in \mathbb{N}$ and every $s \in \Sigma(X)$. Let $s=\left(e_{n}\right)_{n}$ be the basis of $X$. We inductively construct $\left(\mathcal{F}_{n}\right)_{n \in \mathbb{N}} \subset \Sigma(\mathbb{N})$ such that $\mathcal{F}_{n} \succ \mathcal{F}_{n+1}$ for all $n \in \mathbb{N}$, and we have that $\left\|\left(\xi_{n}\right)_{1}^{M}\left(s, \mathcal{G}_{n}\right)\right\|>\frac{\delta}{2}$ for every $\mathcal{G}_{n} \in \Sigma(\mathbb{N})$, every $\mathcal{G}_{n} \subset \mathcal{F}_{n}$, and every $M \in[\mathbb{N}]$.

We show how to construct $\mathcal{F}_{1}, \mathcal{F}_{2}$ and in the same manner we get the general inductive step.

Since $\tau_{\xi_{1}}(s)>\delta$, Lemma [5.6 (2) and Lemma [5.9 yield $\mathcal{G}_{1} \in \Sigma(\mathbb{N})$ and $L_{1} \in[\mathbb{N}]$ such that $S_{\xi_{1}}^{L_{1}} \subset \mathfrak{F}_{\frac{\delta}{2}}\left(s, \mathcal{G}_{1}\right)$. Hence from Lemma 5.11 there exists $\mathcal{F}_{1} \subset \mathcal{G}_{1}$ satisfying the inductive hypothesis for $n=1$. Let $\mathcal{F}_{1}=\left(F_{l}^{1}\right)_{l \in \mathbb{N}}$ and choose $n_{l} \in F_{l}^{1}$. We set $s_{1}=\left(e_{n_{l}}\right)_{l \in \mathbb{N}}$. Since $X$ is stabilized, $\tau_{\xi_{2}}\left(s_{1}\right)>\delta$. Therefore as in the case $n=1$ there exists $\mathcal{G}_{2} \in \Sigma(\mathbb{N})$ and $L_{2} \in[\mathbb{N}]$ such that $S_{\xi_{2}}^{L_{2}} \subset \mathfrak{F}_{\frac{\delta}{2}}\left(s_{1}, \mathcal{G}_{2}\right)$. We show that there exists $\mathcal{G}_{2}^{\prime} \prec \mathcal{F}_{1}$ such that $S_{\xi_{2}}^{L} \subset \mathfrak{F}_{\frac{\delta}{2}}\left(s, \mathcal{G}_{2}^{\prime}\right)$. Indeed, if $\mathcal{G}_{2}=\left(G_{d}\right)_{d \in \mathbb{N}}$ we set $G_{d}^{\prime}=\bigcup_{l \in G_{d}} F_{n_{l}}^{1}$. Then clearly $\mathcal{G}_{2}^{\prime}=\left(G_{d}^{\prime}\right)_{d \in \mathbb{N}} \prec \mathcal{F}_{1}$. Further, if $Y_{G_{d}}=\left\langle e_{n_{l}}: l \in G_{d}\right\rangle$, then $Y_{G_{d}}$ is a subspace of $Y_{G_{d}^{\prime}}=\left\langle e_{n}: n \in G_{d}^{\prime}\right\rangle$. Therefore $\mathfrak{F}_{\frac{\delta}{2}}\left(s_{1}, \mathcal{G}_{2}\right) \subset \mathfrak{F}_{\frac{\delta}{2}}\left(s, \mathcal{G}_{2}^{\prime}\right)$. Hence $S_{\xi_{2}}^{L} \subset \mathfrak{F}_{\frac{\delta}{2}}\left(s, \mathcal{G}_{2}^{\prime}\right)$. Again from Lemma 5.11 there exists $\mathcal{F}_{2} \in \Sigma(\mathbb{N}), \mathcal{F}_{2} \subset \mathcal{G}_{2}^{\prime}$, which satisfies the inductive hypothesis for $n=2$. To go from the $n^{\text {th }}$ to the $(n+1)^{t h}$ step we use exactly the same arguments as in the case from the first step to the second. Therefore the sequence $\left(\mathcal{F}_{n}\right)_{n \in \mathbb{N}}$ has been constructed. Let $\mathcal{F}_{n}=\left(F_{k}^{n}\right)_{k \in \mathbb{N}}$ and set $\mathcal{F}_{\infty}=\left(F_{n}^{n}\right)_{n \in \mathbb{N}}$.

Claim. For every $M \in[\mathbb{N}]$, we have that $\left\|\xi_{1}^{M} \cdot\left(s, \mathcal{F}_{\infty}\right)\right\|>\frac{\delta}{2}$.

It follows immediately that the proof of the claim derives a contradiction since we have assumed that $\tau_{\xi}(s)=\tau_{\xi}(X)=0$. To prove the claim choose any $M=$ $\left(m_{n}\right)_{n \in \mathbb{N}}$. Then from the definition of $\xi_{1}^{M}$ we have that $\xi_{1}^{M}=\left(\xi_{m_{1}}\right)_{1}^{M}$. Since for all $n \geq m_{1}, \mathcal{F}_{n} \prec \mathcal{F}_{m_{1}}$ it follows that there exists $\left(F_{l_{n}}^{m_{1}}\right)_{n \geq m_{1}}$ such that $l_{m_{1}}=m_{1}$ and $F_{l_{n}}^{m_{1}} \subset F_{n}^{n}$. We define $\mathcal{G}=\left\{F_{1}^{m_{1}}, \ldots, F_{m_{1}}^{m_{1}}\right\} \cup\left\{F_{l_{n}}^{m_{1}}\right\}_{n>m_{1}}$. Clearly $\mathcal{G} \subset \mathcal{F}_{m_{1}}$, therefore by the inductive assumption for $L \in[\mathbb{N}]$, we get that $\left\|\left(\xi_{m_{1}}\right)_{1}^{L} \cdot(s, \mathcal{G})\right\|>\frac{\delta}{2}$. In particular for the set $M$, we conclude that $\left\|\left(\xi_{m_{1}}\right)_{1}^{M} \cdot(s, \mathcal{G})\right\|>\frac{\delta}{2}$. If $\mathcal{G}=\left(G_{n}\right)_{n \in \mathbb{N}}$, 
then for $n \geq m_{1}$ we have that $G_{n} \subset F_{n}^{n}$. Hence for the given $M$ it follows readily that $\frac{\delta}{2}<\left\|\left(\xi_{m_{1}}\right)_{1}^{M} \cdot(s, \mathcal{G})\right\| \leq\left\|\left(\xi_{m_{1}}\right)_{1}^{M} \cdot\left(s, \mathcal{F}_{\infty}\right)\right\|$.

This finishes the proof for the limit ordinal case. The case of successor ordinal is proven by similar arguments.

Proposition 5.18. Let $A$ be a stabilized reflexive Banach space with an unconditional basis such that $\xi_{A}$ is a successor ordinal. Then A satisfies property $\left(P_{2}\right)$.

Proof. Let $\xi_{A}=\zeta+1$ and $\tau=\tau_{\zeta}(A)>0$. It is easy to see that also $\tau_{\zeta, n}(A)>0$ for all $n \in \mathbb{N}$. According to Proposition 5.17 we have that $\lim \tau_{\zeta, n}(A)=0$ and so for every $k \in \mathbb{N}$ there exists $n_{k} \in \mathbb{N}$ such that $\tau_{\zeta, n_{k}}(A)<\frac{\tau}{2 k}$.

For every $k \in \mathbb{N}$ we set $C_{k}=n_{k}$. We will prove that for every $\left(x_{i}\right)_{i=1}^{\infty} \in \Sigma(A)$ and $k \in \mathbb{N}$ there exists $t=\left(z_{i}\right)_{i=1}^{\infty} \prec\left(x_{i}\right)_{i=1}^{\infty}$ such that for every $M=\left\{m_{n}\right\}_{n \in \mathbb{N}} \in[\mathbb{N}]$, $\left\|\frac{z_{m_{1}}+\ldots+z_{m_{n_{k}}}}{n_{k}}\right\|<\frac{1}{k}$, which actually implies property $\left(P_{2}\right)$.

Let $s=\left(x_{i}\right)_{i=1}^{\infty} \in \Sigma(A)$ and $B=\left\langle\left(x_{i}\right)_{i=1}^{\infty}\right\rangle$. Since $\tau_{\zeta, n_{k}}(s)=\tau_{\zeta, n_{k}}(A)<\frac{\tau}{2 k}$, for every $\mathcal{F} \in \Sigma(\mathbb{N})$ and $M \in[\mathbb{N}]$ there exists $N \in[M]$ such that if $t \in \Sigma(s, \mathcal{F})$, then $\| \frac{\zeta_{1}^{N}+\ldots+\zeta_{n_{k}}^{N}}{n_{k}} \cdot t||<\frac{\tau}{2 k}$. On the other hand $\tau_{\zeta}(s)=\tau$ and so there exist $\mathcal{F}_{1} \in \Sigma(\mathbb{N})$ and $M_{1} \in[\mathbb{N}]$ such that $\left\|\zeta_{n}^{N}\left(s, \mathcal{F}_{1}\right)\right\|>\frac{\tau}{2}$ for every $N \in\left[M_{1}\right]$ and every $n \in \mathbb{N}$. For every $n \in \mathbb{N}$ we choose $t_{n}=\left(y_{i}^{(n)}\right)_{i} \in \Sigma\left(s, \mathcal{F}_{1}\right)$ such that $\left\|\zeta_{n}^{M_{1}} \cdot t_{n}\right\|>\frac{\tau}{2}$.

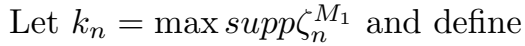

$$
\tilde{s}=\left(\tilde{y}_{i}\right)_{i=1}^{\infty}=\left(y_{1}^{(1)}, \ldots, y_{k_{1}}^{(1)}, y_{k_{1}+1}^{(2)}, \ldots y_{k_{2}}^{(2)}, y_{k_{2}+1}^{(3)}, \ldots\right) .
$$

Then it is readily checked that $\left\|\zeta_{n}^{M_{1}} \cdot \tilde{s}\right\|>\frac{\tau}{2}$ for every $n \in \mathbb{N}$. We set $z_{n}=\frac{\zeta_{n}^{M_{1}} \cdot \tilde{s}}{\left\|\zeta_{n}^{M_{1}} \tilde{s}\right\|}$, $t=\left(z_{n}\right)_{n=1}^{\infty}$.

Let $M \in\left[M_{1}\right], M=\left\{m_{1}, m_{2}, \ldots\right\}$ and set $M_{2}=\bigcup_{k=1}^{\infty} \operatorname{supp} \zeta_{m_{k}}^{M_{1}}$.

Observe that by property (4) of the repeated averages hierarchy we have that $\left\|\zeta_{m_{k}}^{M_{1}} \cdot \tilde{s}\right\|=\left\|\zeta_{k}^{M_{2}} \cdot \tilde{s}\right\|$. Therefore, $\left\|\frac{z_{m_{1}}+\ldots+z_{m_{n_{k}}}}{n_{k}}\right\| \leq \frac{2}{\tau}\left\|\frac{\zeta_{m_{1}}^{M_{1}} \cdot \tilde{s}+\ldots+\zeta_{m_{n_{k}}}^{M_{1}} \cdot \tilde{s}}{n_{k}}\right\|=$ $\frac{2}{\tau}\left\|\frac{\zeta_{1}^{M_{2}} \cdot \tilde{s}+\ldots+\zeta_{n_{k}}^{M_{2}} \cdot \tilde{s}}{n_{k}}\right\|<\frac{1}{k}$, and the proof is complete.

Next we will deal with stabilized Banach spaces $A$ for which $\xi_{A}$ is a limit ordinal. We start with some notation and an auxiliary lemma.

Notation. (a) We recall that for $A$ as above, $X_{A}$ denotes the space defined in the fourth section, and for a finitely supported $x$ in $X_{A}$ we denote by range $(x)$ the set $\{\delta \in \mathfrak{D}: \exists \alpha, \beta \in \operatorname{supp}(x)$ with $|a| \leq|\delta| \leq|\beta|\}$. We write range $(x)<\operatorname{range}(y)$ provided that $\left|\delta_{1}\right|<\left|\delta_{2}\right|$ for every $\delta_{1} \in \operatorname{range}(x)$ and $\delta_{2} \in \operatorname{range}(y)$.

We also let $\Sigma\left(X_{A}\right)=\left\{\left(x_{i}\right)_{i=1}^{\infty}:\left\|x_{i}\right\|=1\right.$ and range $\left.\left(x_{i}\right)<\operatorname{range}\left(x_{i+1}\right)\right\}$.

(b) The definition of $\tau_{\xi}(s)$ for $s \in \Sigma\left(X_{A}\right)$ is the same as in Definition 5.3 We also define $\tau_{\xi}\left(X_{A}\right)=\inf \left\{\tau_{\xi}(s): s \in \Sigma\left(X_{A}\right)\right\}$.

Lemma 5.19. Let $A$ be a stabilized reflexive Banach space with an unconditional basis. Then for every $\xi<\omega_{1}$ and $s \in \Sigma\left(X_{A}\right)$ we have that $\tau_{\xi}(s)=\tau_{\xi}(A)$. 
Proof. Let $s \in \Sigma\left(X_{A}\right), s=\left(x_{i}\right)_{i=1}^{\infty}$. To each $x_{i}=\sum_{|\alpha|=n_{i}}^{m_{i}} \lambda_{\alpha} e_{\alpha}$ we assign a vector $\bar{x}_{i}=\sum_{k=n_{i}}^{m_{i}}\left(\sum_{|\alpha|=k}\left|\lambda_{\alpha}\right|\right) e_{k} \in A$. Clearly $\bar{s}=\left(\bar{x}_{i}\right)_{i=1}^{\infty} \in \Sigma(A)$. Furthermore if $\mathcal{F} \in \sigma(\mathbb{N})$ and $Y_{k}(\mathcal{F}, s)=\operatorname{span}\left[x_{i}: i \in F_{k}\right], \bar{Y}_{k}(\mathcal{F}, s)=\operatorname{span}\left[\bar{x}_{i}: i \in F_{k}\right]$, then $Y_{k}(\mathcal{F}, s)$ is isometric to $\bar{Y}_{k}(\mathcal{F}, s)$. It follows immediately from the definitions that $\tau_{\xi}(s)=\tau_{\xi}(\bar{s})$ and since $A$ is stabilized we get $\tau_{\xi}(s)=\tau_{\xi}(A)$. The proof is complete.

We recall that $W$ denotes the closed symmetric convex subset of $X_{A}$ defined in Section 4.

Proposition 5.20. Let $A$ be a stabilized reflexive Banach space with an unconditional basis such that $\xi_{A}$ is a limit ordinal. Then there exists a null sequence $\mathbf{a}=\left(a_{n}\right)_{n \in \mathbb{N}}$ of positive numbers such that the set $W$ in $X_{A}$ is an $\mathbf{a}$-thin set.

Proof. Since $\tau_{\xi_{A}}\left(X_{A}\right)=0$ it follows from Proposition 5.17 that for the sequence $\left(\xi_{n}\right)_{n \in \mathbb{N}}$ used in the definition of $S_{\xi_{A}}$ we have that $\lim \tau_{\xi_{n}}\left(X_{A}\right)=0$. Therefore for every $k \in \mathbb{N}$ there exists $\xi_{n_{k}}$ such that $\tau_{\xi_{n_{k}}}<\frac{1}{k 2^{k}}$ and we define $a_{k}=\frac{\tau_{\xi n_{k}}}{32 k}$. Our goal is to show that for $\mathbf{a}=\left(a_{k}\right)_{k \in \mathbb{N}}, W$ is an a-thin set.

Assume on the contrary that this is not the case. Then there exists an infinitedimensional closed subspace $Z$ of $X_{A}$ and $\lambda>0$ such that for all $n \in \mathbb{N}, B_{Z} \subset$ $\lambda\left(2^{k} W+a_{k} B_{X_{A}}\right)$.

Choose $k>1000 \lambda$ and for the rest of the proof we will denote by $\zeta$ the ordinal $\xi_{n_{k}}$ and by $\tau$ the positive real number $\tau_{\xi_{n_{k}}}(A)$. We may also assume that $Z$ is a block subspace generated by a sequence $\left(z_{l}\right)_{l \in \mathbb{N}}$ with range $\left(z_{l}\right)<$ range $\left(z_{l+1}\right)$.

Given $\mathcal{F} \in \Sigma(\mathbb{N}), \mathcal{F}=\left(F_{m}\right)_{m \in \mathbb{N}}$, we consider the following finite-dimensional spaces. The first is the space $Z_{F_{m}}=\operatorname{span}\left[z_{l}: l \in F_{m}\right]$ and the second is $X_{F_{m}}=$ $\operatorname{span}\left[\left\{e_{\delta}: \delta \in \bigcup_{l \in F_{m}} \operatorname{range}\left(z_{l}\right)\right\}\right]$. Clearly $Z_{F_{m}} \subset X_{F_{m}}$.

We start by showing the following:

$\left.{ }^{*}\right)$ For every $\mathcal{F} \in \Sigma(\mathbb{N})$ and $M \in[\mathbb{N}]$ there exists $N \in[M]$ such that for every $L \in[N]$ and $t=\left(t_{m}\right)$ with $t_{m} \in X_{F_{m}},\left\|t_{m}\right\|=1$, we have that $\left\|\zeta_{1}^{L} \cdot t\right\| \leq 2 \tau$.

Indeed, as in the proof of the previous lemma, to each such $t$ we assign $\bar{t} \in$ $\Sigma(s, \mathcal{F})$ where $s=\left(e_{l}\right)_{l \in \mathbb{N}}$ is the basis of $A$. Moreover, as observed there, we have $\left\|\zeta_{1}^{L} \cdot t\right\|=\left\|\zeta_{1}^{L} \cdot \bar{t}\right\|_{A}$. Since $\tau_{\xi}(s)=\tau$, we have that for every $\mathcal{F} \in \Sigma(\mathbb{N})$ and $M \in[\mathbb{N}]$ there exists $N \in[M]$ such that $\max \left\{\left\|\zeta_{1}^{L} \cdot s^{\prime}\right\|_{A}: s^{\prime} \in \Sigma\left(s^{\prime}, \mathcal{F}\right)\right\} \leq 2 \tau$ for every $L \in[N]$ (Lemma 5.6 (1)). Then for $L \in[N]$,

$$
\begin{aligned}
\max & \left\{\left\|\zeta_{1}^{L} \cdot t\right\|_{X_{A}}: t=\left(t_{m}\right), t_{m} \in X_{F_{m}},\left\|t_{m}\right\|=1\right\} \\
& \leq \max \left\{\left\|\zeta_{1}^{L} \cdot \bar{t}\right\|_{A}: t=\left(t_{m}\right), t_{m} \in X_{F_{m}},\left\|t_{m}\right\|=1\right\} \\
& \leq \max \left\{\left\|\zeta_{1}^{L} \cdot s^{\prime}\right\|_{A}: s^{\prime} \in \Sigma(s, \mathcal{F})\right\} \leq 2 \tau .
\end{aligned}
$$

This completes the proof of $(*)$.

Since the space $A$ is stabilized from Lemma 5.19 we get that, for every $s \in \Sigma\left(X_{A}\right)$ and $\xi<\omega_{1}, \tau_{\xi}(s)=\tau_{\xi}(A)$. Therefore for the sequence $t=\left(z_{l}\right)_{l \in \mathbb{N}}$ there exists $\mathcal{F}=\left(F_{m}\right)_{m}$, satisfying the following property: 
There exists $M \in[\mathbb{N}]$ such that for all $N \in[M]$,

$$
\left\|\zeta_{1}^{N}(t, \mathcal{F})\right\|>\frac{1}{2} \tau
$$

According to Lemma 5.9 there exists $M_{1} \in[M]$ such that $S_{\zeta}^{M_{1}} \subset \mathfrak{F}_{\frac{\tau}{2}}(t, \mathcal{F})$. Furthermore if $\mathcal{G}=\left(F_{m}\right)_{m \in M_{1}}$, then $S_{\zeta} \subset \mathfrak{F}_{\frac{\tau}{4}}(t, \mathcal{G})$ (Lemma 5.11). Therefore we may assume that the family $\mathcal{F}$ and the set $M$ satisfy the following stronger property:

For every $N \in[M]$ there exists $x^{*} \in B_{X_{A}^{*}}$ with $\left\|x^{*} \mid Z_{F_{m}}\right\|>\tau / 4 \quad \forall m \in \operatorname{supp} \zeta_{1}^{N}$.

We choose $N \in[M]$ such that condition $\left(^{*}\right)$ is also fulfilled.

Consider the following family of finite subsets of $\mathbb{N}$ :

$$
\mathfrak{F}=\left\{\begin{array}{c}
G \subset \mathbb{N}: \exists x_{G}^{*} \in B_{X_{A}^{*}} \text {, and }\left(y_{m}\right)_{m \in G} \text { such that } \\
\text { (a) } y_{m} \in Z_{F_{m}} \text { and }\left\|y_{m}\right\| \leq 1 \\
\text { (b) } \forall m \exists E_{m}^{\prime} \subset \text { rangey } \\
\text { and the sets }\left(E_{m}^{\prime}\right)_{m \in G} \text { are pairwise incomparable } \\
\text { (c) }\left\|\left.x_{G}^{\prime}\right|_{X_{F}}\right\|<8 \tau
\end{array}\right\} .
$$

Claim. There exists $\varepsilon>0$ such that $\mathfrak{F}$ is $(\zeta, N, \varepsilon)$-large.

Suppose that the claim has been proved. Then we derive a contradiction in the following manner.

By Theorem 5.4 and Lemma 5.1 (see also Corollary [5.5) there exists an $L \in[N]$ such that if $Q \in[L]$, then the set $G^{Q}=\operatorname{supp} \zeta_{1}^{N} \backslash\left\{\min \operatorname{supp} \zeta_{1}^{N}\right\}$ belongs to $\mathfrak{F}$. By property (1) of the repeated averages hierarchy we can find $Q \in[L]$ such that

$$
\sum_{m \in G^{Q}} \zeta_{1}^{Q}(m)>\frac{1}{2}
$$

Since $G^{Q} \in \mathfrak{F}$, there exist a functional $x^{*}=x_{G^{Q}}^{*} \in B_{X_{A}^{*}}$ and a sequence $\left(y_{m}\right)_{m \in G^{Q}}$ satisfying conditions (a), (b), (c). We set $\tilde{y}=\sum_{m \in G^{Q}} \zeta_{1}^{Q}(m) y_{m}, y=$ $\sum_{m \in G^{Q}} \zeta_{1}^{Q}(m) E_{m}^{\prime} y_{m}$

Choose $\tilde{w} \in W_{0}$ such that \|\|$\tilde{y}\left\|^{-1} \tilde{y}-\lambda \cdot 2^{k} \tilde{w}\right\|<\lambda a_{k}$ and set $w_{m}=E_{m}^{\prime} \tilde{w}, w=$ $\sum_{m \in G^{Q}} w_{m}, u=\|\tilde{y}\|^{-1} y-\lambda \cdot 2^{k} w$. Then

$$
\|u\|=\|\| \tilde{y}\left\|^{-1} y-\lambda \cdot 2^{k} w\right\|<\lambda a_{k} .
$$

By (1) we get that

$$
\|\tilde{y}\|<2 \tau,
$$

and by (2) and condition (b) of the definition of $\mathfrak{F}$

$$
x^{*}(y)>\frac{\tau}{32} .
$$

Since the sets $\left(E_{m}^{\prime}\right)_{m \in G^{Q}}$ are pairwise incomparable we get that $w$ is a convex combination of vectors $w_{m}^{\prime} \in B_{X_{F_{m}}}, m \in G^{Q}$. Therefore by property (c) of the definition of the family $\mathfrak{F}$ we have that

$$
x^{*}(w) \leq 8 \tau .
$$

Combining relations (4), (5) and (6) above, we obtain that $x^{*}(u)>\frac{1}{2 \tau} \frac{\tau}{32}-$ $2^{k} \lambda 8 \tau>\lambda a_{k}$ which contradicts (3).

Hence it remains to prove the claim. 
For any $L \in[N]$ set $G=\operatorname{supp} \zeta_{n}^{L}$ and choose $x^{*} \in B_{X_{A}^{*}}$ such that $\left\|\left.x^{*}\right|_{Z_{F m}}\right\|>\frac{\tau}{4}$ for all $m \in G$.

We write $x^{*}=\left(x^{*}\right)^{+}-\left(x^{*}\right)^{-}$, where $\left(x^{*}\right)^{+}\left(e_{\delta}\right)=\left\{\begin{array}{ll}x^{*}\left(e_{\delta}\right) & \text { if } x^{*}\left(e_{\delta}\right)>0 \\ 0 & \text { otherwise }\end{array}\right.$ and $\left(x^{*}\right)^{-}\left(e_{\delta}\right)=\left\{\begin{array}{ll}-x^{*}\left(e_{\delta}\right) & \text { if } x^{*}\left(e_{\delta}\right)<0 \\ 0 & \text { otherwise }\end{array}\right.$.

We set $G_{+}=\left\{m \in G:\left\|\left.\left(x^{*}\right)^{+}\right|_{Z_{m}}\right\|>\frac{\tau}{8}\right\}, G_{-}=\left\{m \in G:\left\|\left.\left(x^{*}\right)^{-}\right|_{Z_{F m}}\right\|>\frac{\tau}{8}\right\}$. Then either $\sum_{m \in G_{+}} \zeta_{1}^{L}(m)>\frac{1}{2}$ or $\sum_{m \in G_{-}} \zeta_{1}^{L}(m)>\frac{1}{2}$.

Suppose that

$$
\sum_{m \in G_{+}} \zeta_{1}^{L}(m)>\frac{1}{2}
$$

and set $z^{*}=\left(x^{*}\right)^{+}, G^{1}=\left\{m \in G_{+}:\left\|\left.z^{*}\right|_{X_{F_{m}}}\right\|<8 \tau\right\}$. Since for each $t=\left(t_{m}\right)_{m \in G}$, $t_{m} \in X_{F_{m}},\left\|t_{m}\right\|=1$ we have $\left\|\zeta_{1}^{L} \cdot t\right\|<2 \tau$, (17) yields that

$$
\sum_{m \in G^{1}} \zeta_{1}^{L}(m)>\frac{1}{4}
$$

Further choose

$$
y_{m} \in Z_{F_{m}} \text { such that }\left\|y_{m}\right\|=1 \text { and } x^{*}\left(y_{m}\right)>\frac{\tau}{4} .
$$

For every $m \in G^{1}$ choose $w_{m} \in W$ such that

$$
\left\|y_{m}-\lambda 2^{k} w_{m}\right\|<\lambda a_{k} .
$$

Set $\varepsilon_{1}=\frac{1}{32} \frac{\tau}{2^{k} \lambda}$ and $E_{m}=$ range $\left(y_{m}\right)$. We apply Proposition 4.4 to find a partition $D_{1}, \ldots, D_{p}$ of the set $G$ and $E_{m}^{\prime} \subset E_{m}$ such that $(i) z^{*}\left(\left(E_{m} \backslash E_{m}^{\prime}\right) w_{m}\right)<\varepsilon_{1}$, (ii) the sets $\left(E_{m}^{\prime}\right)_{m \in D_{i}}$ are pairwise incomparable for every $i=1, \ldots, p$ and (iii) $p<\frac{5}{\varepsilon_{1}^{2}}$. By (8) there exists $i_{0} \in\{1, \ldots, p\}$ such that $\sum_{m \in D_{i_{0}}} \zeta_{1}^{N}(m)>\frac{1}{4 p}>\frac{\varepsilon_{1}^{2}}{20}$. By (9), (10) and (i) we have that $z^{*}\left(E_{m}^{\prime} y_{m}\right)>\frac{\tau}{8}-2^{k} \lambda \varepsilon_{1}-\lambda a_{k}>\frac{\tau}{8}-\frac{1}{32} \frac{\tau}{2^{k} \lambda}-\lambda \frac{\tau}{32 k}>\frac{\tau}{16}$. Therefore $D_{i_{0}} \in \mathfrak{F}$. Hence for $\varepsilon=\frac{\varepsilon_{1}^{2}}{20}$ the set $\mathfrak{F}$ is $(\zeta, N, \varepsilon)$-large as claimed.

This completes the entire proof.

Theorem 5.21. Let $A$ be a reflexive Banach space with an unconditional basis. Then there exists a block subspace $B$ and a null sequence a such that the set $W$ in $X_{B}$ is an a-thin set.

Proof. From Proposition 5.16 we choose a stabilized block subspace $B$ of the space $A$. Then if $\xi_{B}$ is a successor ordinal, then the result follows from Proposition 5.18 while if $\xi_{B}$ is a limit ordinal, then it follows from Proposition 5.20.

\section{Thin NORMing SETS III $\left(c_{0}(\mathbb{N})\right.$ CASE $)$}

In this section for $A=c_{0}(\mathbb{N})$ we construct a Banach space $X_{A}$ isometric to $\left(\sum_{n=1}^{\infty} \oplus \ell^{1}\left(2^{n}\right)\right)_{0}$ and a set $W$ which norms a subspace of $X_{A}^{*} w^{*}$-isometric to $\left(c_{0}(\mathbb{N})\right)^{*}$. The set $W$ is in a way similar to the reflexive space case. The proof that $W$ is a thin set uses the structure of the $w^{*}$-closure of $W$ in the second dual of $X_{A}$. 
Theorem 6.1. There exists a Banach space $X_{c_{0}}$ with separable dual $X_{c_{0}}^{*}$ and a closed, bounded convex symmetric subset $W$ of $X_{c_{0}}$ such that $W$ is a thin subset of $X_{c_{0}}, c_{0}(\mathbb{N})^{*}$ is $w^{*}$-isometric to a closed subspace $Y$ of $X_{c_{0}}^{*}$ and $W$ is a $\frac{1}{2}$-norming set for $Y$.

We define the spaces $X_{c_{0}}$ and $Y$ in a manner similar to the reflexive case.

We denote by $\mathfrak{D}$ the dyadic tree, i.e. every $\delta \in \mathfrak{D}$ has exactly two immediate successors. The tree $\mathfrak{D}$ naturally coincides with the set of all finite sequences $\left\{\left(\varepsilon_{1}, \ldots, \varepsilon_{n}\right): n \in \mathbb{N}\right.$ and $\varepsilon_{i} \in\{0,1\}$, for $\left.i=1,2, \ldots, n\right\}$. We will use the notation and the definitions about the tree $\mathfrak{D}$ introduced in Section 4 . In the vector space $c_{00}(\mathfrak{D})$ we define the following norm: $\left\|\sum_{\delta \in \mathfrak{D}} \lambda_{\delta} e_{\delta}\right\|=\max _{n \in \mathbb{N}}\left(\sum_{|\delta|=n}\left|\lambda_{\delta}\right|\right)$.

The space $X_{c_{0}}$ is the completion of $c_{00}(\mathfrak{D})$ with the above defined norm. It is clear that $X_{c_{0}}$ is isometric to the space $\left(\sum_{n \in \mathbf{N}} \oplus \ell^{1}\left(2^{n}\right)\right)_{0}$. The space $X_{c_{0}}^{*}$ is isometric to the space $\left(\sum_{n \in \mathbf{N}} \oplus \ell^{\infty}\left(2^{n}\right)\right)_{1}$ and $X_{c_{0}}^{* *}$ is isometric to $\left(\sum_{n \in \mathbf{N}} \oplus \ell^{1}\left(2^{n}\right)\right)_{\infty}$. The last space is not a $d$-product of $\left(\ell^{1}\left(2^{n}\right)\right)_{n \in \mathbb{N}}$.

The space $Y$ is defined as follows: We set $y_{n}^{*}=\sum_{|\delta|=n, \delta(n)=1} e_{\delta}^{*}$, where $\delta=$ $\left(\varepsilon_{1}, \ldots, \varepsilon_{n}\right), \delta(n)=\varepsilon_{n}$, and $Y=\overline{\operatorname{span}}\left[\left(y_{n}^{*}\right)_{n \in \mathbb{N}}\right]$. It is easy to see that the space $Y$ is $w^{*}$-isometric to $c_{0}^{*}(\mathbb{N})$. Next we define the set $W$. For any initial segment $s$ of $\mathfrak{D}$ we let $x_{s}=\sum_{\delta \in s} e_{\delta}$. Clearly, $\left\|x_{s}\right\|=1$.

We set $W=\overline{c o}\left\{ \pm x_{s}: s\right.$ is an initial segment $\}$. For any infinite branch $\gamma$ of $\mathfrak{D}$ we set $x_{\gamma}=w^{*}-\sum_{\delta \in \gamma} e_{\delta}=w^{*}-\lim _{n \rightarrow \infty} x_{\left.\gamma\right|_{n}}$. Clearly each $x_{\gamma} \in X_{c_{0}}^{* *} \backslash X_{c_{0}}$. It remains to prove the following:

Proposition 6.2. The set $W$ is $\frac{1}{2}$-norming for the space $Y$ and it is a thin subset of $X_{c_{0}}$.

Proof. The conclusion that the set $W$ is a $\frac{1}{2}$-norming set can be proved by similar arguments as in Lemma 4.1. It remains to be shown that $W$ is a thin subset of $X_{c_{0}}$.

Assume on the contrary that $W$ is not a thin set. Then for every $\varepsilon>0$ there exists a normalized block sequence $\left(z_{n}\right)_{n \in \mathbb{N}}$ in $X$ and $\lambda>0$ such that $B_{Z} \subset \lambda W+\varepsilon B_{X_{c_{0}}}$, where $Z=\overline{\left\langle\left(z_{n}\right)_{n \in \mathbb{N}}\right\rangle}$. We denote by $\tilde{W}$ the $w^{*}$-closure of $W$ in $X_{c_{0}}^{* *}$ and by the compactness of $\tilde{W}$ in the $w^{*}$-topology we get that $B_{Z^{* *}} \subset \lambda \tilde{W}+\varepsilon B_{X_{c_{0}}^{* *}}$. We denote by $\operatorname{Br}(\mathfrak{D}), S(\mathfrak{D})$ the sets of infinite branches and finite initial segments of $\mathfrak{D}$ respectively. Define $K_{c}=\left\{x_{\gamma}: \gamma \in B r(\mathfrak{D})\right\}, K_{d}=\left\{x_{s}: s \in S(\mathfrak{D})\right\}$ and $K=$ $K_{c} \cup K_{d}$. It is easy to check that $\tilde{W}=\overline{c o}^{w^{*}}(K \cup-K)$. Finally $\mathcal{M}\left(K_{c}\right)$ denotes the space of the regular finite Borel measures on $K_{c}, \mathcal{M}_{1}\left(K_{c}\right)=\{\mu \in \mathcal{M}(K):\|\mu\| \leq 1\}$ and $Q: X_{c_{0}}^{* *} \rightarrow X_{c_{0}}^{* *} / X_{c_{0}}$ is the natural quotient map. We will use the following three lemmas.

Lemma 6.3. If $R: \mathcal{M}_{1}\left(K_{c}\right) \rightarrow \tilde{W}$ is the natural affine map from the unit ball of the Borel measures on $K_{c}$ onto $\tilde{W}$, then $Q R$ is an isometry between $\mathcal{M}_{1}\left(K_{c}\right)$ and $Q(\tilde{W})$. 
Proof. Notice first that $\left(K_{c}, w^{*}\right)$ is a compact metric space homeomorphic to the Cantor set $\{0,1\}^{\mathbb{N}}$. Hence the family $\left(\mathcal{N}_{\delta}\right)_{\delta \in \mathfrak{D}}$, where $\mathcal{N}_{\delta}=\left\{x_{\gamma}: \delta \in \gamma\right\}$, is a basis for the topology of $K_{c}$ and each $\mathcal{N}_{\delta}$ is a clopen subset of $K_{c}$. Now for $\mu \in \mathcal{M}_{1}\left(K_{c}\right)$, $\|\mu\|=\lim _{n \rightarrow \infty} \sum_{|\delta|=n}\left|\mu\left(\mathcal{N}_{\delta}\right)\right|=\lim _{n \rightarrow \infty} \sum_{|\delta|=n}\left|R \mu\left(e_{\delta}\right)\right|=d\left(R \mu, X_{c_{0}}\right)=\|Q R \mu\|$ and this completes the proof of the lemma.

Let $\left\{\mu_{i}\right\}_{i \in I} \subset \mathcal{M}_{1}\left(K_{c}\right)$ and $\delta>0$. We say that $\left\{\mu_{i}\right\}_{i \in I}$ is $\delta$-singular if there exists $\left\{\nu_{i}\right\}_{i \in I} \subset \mathcal{M}_{1}\left(K_{c}\right)$ such that $\left\|v_{i}\right\|<\delta$ and $\left\{\mu_{i}-\nu_{i}\right\}_{i \in I}$ is a pairwise singular family.

Lemma 6.4. Let $\left(\mu_{\xi}\right)_{\xi \in \Xi}$ be an uncountable family of measures in $\mathcal{M}_{1}\left(K_{c}\right)$. Then for any $\delta>0$ there exists an uncountable family $\left\{\left\{\xi_{i}, \zeta_{i}\right\}, i \in I\right\}$ of pairwise disjoint two-point sets such that $\left\{\mu_{\xi_{i}}-\mu_{\zeta_{i}}, i \in I\right\}$ is $\delta$-singular.

Proof. It is well known $\left([\underline{\mathrm{L}})\right.$ that $\mathcal{M}_{1}\left(K_{c}\right)$ is isometric to $\left(\sum_{\alpha<2^{w}} \oplus L^{1}\left(\mu_{\alpha}\right)\right)_{1}$ where $\left\{\mu_{\alpha}\right\}_{\alpha<2^{w}}$ is a maximal family of pairwise singular probability measures in $\mathcal{M}_{1}\left(K_{c}\right)$. The isometry assigns the vector $\left\{\frac{d \mu}{d \mu_{\alpha}}\right\}_{\alpha<2^{w}}$ to each $\mu \in \mathcal{M}\left(K_{c}\right)$. Since $K_{c}$ is a compact metric space, each $L^{1}\left(\mu_{\alpha}\right)$ is a separable Banach space. Given $\delta>0$, for each $\xi \in \Xi$ we choose a finite subset $F_{\xi}$ of $2^{w}$ such that $\left\|\mu_{\xi}-\sum_{\alpha \in F_{\xi}} \frac{d \mu_{\xi}}{d \mu_{\alpha}}\right\|<\frac{\delta}{4}$. Apply the Erdös-Rado theorem ([KM] $)$ to get an uncountable subset $\Xi^{\prime}$ of $\Xi$ and a subset $F$ of $2^{\omega}$ such that for $\xi_{1}, \xi_{2} \in \Xi^{\prime}, \xi_{1} \neq \xi_{2}$, we have $F_{\xi_{1}} \cap F_{\xi_{2}}=F$. If $F=\emptyset$, then the measures $\left(\mu_{\xi}\right)_{\xi \in \Xi^{\prime}}$ are pairwise $\frac{\delta}{4}$-singular, hence for any family $\left\{\left\{\xi_{i}, \zeta_{i}\right\}, i \in I\right\}$ of pairwise disjoint two-point subset of $\Xi^{\prime}$, the measures $\left\{\mu_{\xi_{i}}-\mu_{\zeta_{i}}\right\}_{i \in I}$ are pairwise $\frac{\delta}{2}$-singular. Therefore we assume that $F \neq \emptyset$. We set $\tau_{\xi}=\sum_{\alpha \in F} \frac{d \mu_{\xi}}{d \mu_{\alpha}}$ for any $\xi \in \Xi^{\prime}$. Since $\left(\sum_{\alpha \in F} \oplus L^{1}\left(\mu_{\alpha}\right)\right)_{1}$ is a separable Banach space there exists an uncountable family $\left\{\left\{\xi_{i}, \zeta_{i}\right\}\right\}_{i \in I}$ of pairwise disjoint two-point subsets of $\Xi^{\prime}$ such that for each $i \in I,\left\|\tau_{\xi_{i}}-\tau_{\zeta_{i}}\right\|<\frac{\delta}{4}$. It is easy to check that the members of the family $\left\{\mu_{\xi_{i}}-\mu_{\zeta_{i}}\right.$ : $i \in I\}$ are pairwise $\delta$-singular.

In the sequel by a normalized block sequence in the space $X_{c_{0}}$ we understand a sequence $\left(z_{n}\right)_{n \in \mathbb{N}}$ of vectors of $X_{c_{0}}$ with finite supports $\left(E_{n}\right)_{n \in \mathbb{N}}$ and such that

$$
\max \left\{|\delta|: \delta \in E_{n}\right\}<\min \left\{|\delta|: \delta \in E_{n+1}\right\} .
$$

Lemma 6.5. Let $\left(z_{n}\right)_{n \in \mathbb{N}}$ be a normalized block sequence in the space $X_{c_{0}}$ and $\left\{M_{\xi}\right\}_{\xi \in \Xi}$ an uncountable almost disjoint family of subsets of $\mathbb{N}$. For any $\xi \in \Xi$ set $z_{\xi}^{* *}=\sum_{n \in M_{\xi}} z_{n}$, in the $w^{*}$-sense. Then the family $\left\{Q z_{\xi}^{* *}\right\}_{\xi \in \Xi}$ is isometrically equivalent to the usual basis of $c_{0}(\Xi)$.

Proof. Since $\left(M_{\xi}\right)_{\xi \in \Xi}$ are almost disjoint and $\left(z_{n}\right)_{n \in \mathbb{N}}$ is a normalized block sequence, then $\left\|\sum_{i=1}^{n} \lambda_{i} Q z_{\xi_{i}}^{* *}\right\|=\lim _{n \rightarrow \infty}\left\|\sum_{i=1}^{n} \lambda_{i} z_{\xi_{i}}^{* *} \mid\{\delta:|\delta| \geq n\}\right\|=\max \left\{\left|\lambda_{i}\right|: 1 \leq i \leq\right.$ $n\}$.

Completion of the proof of Proposition 6.2. For $\varepsilon=\frac{1}{16}$ choose a normalized block sequence $\left(z_{n}\right)_{n \in \mathbb{N}}$ and $\lambda>0$ such that $B_{Z} \subset \lambda W+\varepsilon B_{X_{c_{0}}}$, where $Z=\overline{\operatorname{span}}\left[\left(z_{n}\right)_{n \in \mathbb{N}}\right]$. 
Let $\left(N_{\xi}\right)_{\xi \in \Xi}$ be an uncountable almost disjoint family of subsets of $\mathbb{N}$ and for any $\xi \in \Xi$ define $z_{\xi}^{* *}=\sum_{n \in N_{\xi}} z_{n}$. Since $\left(z_{\xi}^{* *}\right)_{\xi \in \Xi}$ is a subset of $B_{Z^{* *}}$ there exists a family $\left\{\mu_{\xi}\right\}_{\xi \in \Xi}$ in $\mathcal{M}_{1}\left(K_{c}\right)$ such that $\left\|\lambda Q R \mu_{\xi}-Q z_{\xi}^{* *}\right\|<\varepsilon$. Since $\left\|Q z_{\xi}^{* *}\right\|=1$, it follows that $1-\varepsilon<\left\|\lambda \mu_{\xi}\right\|=\left\|\lambda Q R \mu_{\xi}\right\|<1+\varepsilon$.

By Lemma 6.4 we get an uncountable family $\left\{\lambda \mu_{\xi_{i}}-\lambda \mu_{\zeta_{i}}\right\}_{i \in I}$ which is $\frac{1}{16}$ singular. Since $\left\|Q\left(z_{\xi_{i}}^{* *}-z_{\zeta_{i}}^{* *}\right)\right\|=1$, we get that $1-2 \varepsilon<\left\|\lambda Q L\left(\mu_{\xi_{i}}-\mu_{\zeta_{i}}\right)\right\|<$ $1+2 \varepsilon$ and also we have that $\left\|\lambda Q L\left(\mu_{\xi_{i}}-\mu_{\zeta_{i}}\right)-\left(z_{\xi_{i}}^{* *}-z_{\zeta_{i}}^{* *}\right)\right\|<2 \varepsilon$. Hence, $1=$ $\left\|\sum_{i=1}^{n} Q\left(z_{\xi_{i}}^{* *}-z_{\zeta_{i}}^{* *}\right)\right\| \geq\left\|\sum_{i=1}^{n} \lambda Q R\left(\mu_{\xi_{i}}-\mu_{\zeta_{i}}\right)\right\|-2 \varepsilon \geq(1-2 \varepsilon-2 \delta) n-2 \varepsilon \geq\left(1-\frac{1}{4}\right) n-\frac{2}{4}$ which leads to a contradiction for $n \geq 4$. The proof of the proposition is complete. Theorem 6.1 follows immediately from Proposition 6.2.

\section{Constructions of Block-H.I. $d$-Products}

In this section we proceed to the construction of block-H.I. $d$-product norms. The section is divided into two parts. The first is devoted to a brief presentation of mixed Tsirelson $d$-product spaces and the second to the construction of a GowersMaurey type block-H.I. norm. The norm defined here is an adaptation of the Gowers-Maurey definition. We follow closely their proof and there are only a few points where we proceed in a different manner.

7.1. Mixed Tsirelson $d$-products. We start by recalling that for $\tilde{x}=\left(x_{n}\right)_{n=1}^{\infty} \in$ $\Omega_{00}=\left(\prod_{n=1}^{\infty} X_{n}\right)_{00}$ we have denoted by $\operatorname{supp}(\tilde{x})$ the set $\left\{n \in \mathbb{N}: x_{n} \neq 0\right\}$, by range $(\tilde{x})$ the interval of $\mathbb{N}[\min \operatorname{supp}(\tilde{x}), \max \operatorname{supp}(\tilde{x})]$, and by $E \tilde{x}$ the vector $P_{E}(\tilde{x})$ for $E \subset \mathbb{N}$. We also use the notation $\tilde{x}_{1}<\tilde{x}_{2}$ to denote blocks $\tilde{x}_{1}, \tilde{x}_{2}$ with $\operatorname{supp}\left(\tilde{x}_{1}\right)<\operatorname{supp}\left(\tilde{x}_{2}\right)$.

Let $\mathcal{M}$ be an adequate family of finite subsets of $\mathbb{N}$. A sequence $\left(E_{1}, \ldots, E_{n}\right)$ of finite subsets of $\mathbb{N}$ is called $\mathcal{M}$-admissible if there exists a set $\left\{m_{1}, \ldots, m_{n}\right\} \in \mathcal{M}$ such that $m_{1} \leq E_{1}<m_{2} \leq E_{2}<\ldots<m_{n} \leq E_{n}$. A sequence $\left(\tilde{x}_{1}, \ldots, \tilde{x}_{n}\right)$ of successive vectors of $\Omega_{00}=\left(\prod_{n=1}^{\infty} X_{n}\right)_{00}$ is called $\mathcal{M}$-admissible if the sequence ( supp $\tilde{x}_{1}, \ldots$, supp $\left.\tilde{x}_{n}\right)$ is $\mathcal{M}$-admissible.

Let $\theta \in \mathbb{R}$ and $\mathcal{M} \subset[\mathbb{N}]^{<\omega}$. A subset $\mathcal{K}$ of $\Omega_{00}$ is called $(\theta, \mathcal{M})$-closed if for any $\mathcal{M}$-admissible sequence $\left(\tilde{x}_{1}, \ldots, \tilde{x}_{n}\right)$ of elements of $\mathcal{K}$ the vector $\theta \sum_{i=1}^{n} \tilde{x}_{i} \in \mathcal{K}$.

Proposition 7.1. Let $\left(X_{n},\|\cdot\|\right)_{n \in \mathbb{N}}$ be a sequence of Banach spaces. For every $n \in \mathbb{N}$ we choose $\mathcal{F}_{n} \subseteq X_{n}^{*} \subset \Omega_{00}^{*}=\left(\prod_{n=1}^{\infty} X_{n}^{*}\right)_{00}$. Then for every sequence $\left(\theta_{k}, \mathcal{M}_{k}\right)_{k=1}^{\infty}$ there exists a smallest set $\mathcal{K} \subset \Omega_{00}^{*}$ containing $\bigcup_{n=1}^{\infty} \mathcal{F}_{n}$ and closed under the $\left(\theta_{k}, \mathcal{M}_{k}\right)$-operation for all $k \in \mathbb{N}$. Furthermore if each $\mathcal{F}_{n}$ is countable so is $\mathcal{K}$.

Proof. To define the set $\mathcal{K}$ we proceed by induction. For $l=0$ we set $\mathcal{K}_{0}=\bigcup_{n=1}^{\infty} \mathcal{F}_{n}$. If $\mathcal{K}_{l}$ has been defined we set

$$
\mathcal{K}_{l+1}=\left\{\theta_{k} \sum_{i=1}^{d} \phi_{i}:\left(\phi_{i}\right)_{i=1}^{d} \subset \mathcal{K}_{l} \text { is } \mathcal{M}_{k} \text {-admissible, } k \in \mathbb{N}\right\} \cup \mathcal{K}_{l} .
$$


Finally, we set $\mathcal{K}=\bigcup_{l=0}^{\infty} \mathcal{K}_{l}$. It is easily verified that $\mathcal{K}$ is closed under the $\left(\theta_{k}, \mathcal{M}_{k}\right)$ operation for all $k \in \mathbb{N}$ and moreover any other $\mathcal{K}^{\prime}$ having this property and containing $\bigcup_{n=1}^{\infty} \mathcal{F}_{n}$ contains $\mathcal{K}$ as well. Hence $\mathcal{K}$ is the smallest set with these properties. Also if $\bigcup_{n=1}^{\infty} \mathcal{F}_{n}$ is countable, then inductively we show that every $\mathcal{K}_{l}$ is countable, hence $\mathcal{K}$ is countable.

Let $\left(\theta_{k}, \mathcal{M}_{k}\right)_{k=1}^{\infty}$ be such that each $\mathcal{M}_{k}$ is a compact and adequate family of finite subsets of $\mathbb{N}$ and $0<\theta_{k}<1, \lim _{k} \theta_{k}=0$.

Definition 7.1. Let $\left(X_{n}\right)_{n=1}^{\infty}$ be a sequence of Banach spaces and choose $\mathcal{F}_{n} \subset$ $B_{X_{n}^{*}}$ a symmetric and norming set. We denote by $\mathcal{K}$ the set resulting from Proposition 7.1 applied on the families $\left\{\mathcal{F}_{n}\right\}_{n=1}^{\infty},\left\{\left(\theta_{k}, \mathcal{M}_{k}\right)\right\}_{k=1}^{\infty}$. Then the $\left(\theta_{k}, \mathcal{M}_{k}\right)_{k=1}^{\infty}$ mixed Tsirelson $d$-product norm on $\Omega_{00}$ is defined by:

$$
\|\tilde{x}\|=\sup \{\phi(\tilde{x}): \phi \in \mathcal{K}\} .
$$

Remark 7.1. (i) If $X_{n}=\mathbb{R}$ and $\mathcal{F}_{n}=\left\{e_{n},-e_{n}\right\}$, then the above definition coincides with the mixed Tsirelson spaces introduced in [AD1.

(ii) An alternative description of the $\left(\theta_{k}, \mathcal{M}_{k}\right)_{k=1}^{\infty}$ norm \|\| is given by the implicit formula

$$
\|\tilde{x}\|=\max \left\{\|\tilde{x}\|_{\infty}, \sup _{k \in \mathbb{N}}\left\{\sup \theta_{k} \sum_{i=1}^{d}\left\|E_{i} \tilde{x}\right\|\right\}\right\}
$$

where $\|\tilde{x}\|_{\infty}=\max \left\{\left\|x_{n}\right\|_{n}: n \in \mathbb{N}\right\}$ and the inside sup is taken over all $\mathcal{M}_{k}$-admissible sequences $\left(E_{1}, \ldots, E_{n}\right)$.

(iii) Most of the already existing examples use the following two collections of compact adequate families. The first is $\left(\mathcal{A}_{k}\right)_{k \in \mathbb{N}}$ where $\mathcal{A}_{k}=\{F \subset \mathbb{N}:|F| \leq k\}$, and the second is $\left(\mathcal{S}_{k}\right)_{k \in \mathbb{N}}$ where $\mathcal{S}_{k}$ is the Schreier family defined in Section 5 . Thus the Schlumprecht space $S$ is defined by $\left(\frac{1}{\log _{2}(k+1)}, \mathcal{A}_{k}\right)_{k=1}^{\infty}$ and the ArgyrosDeliyanni spaces are built with the use of families of the form $\left(\frac{1}{m_{k}}, \mathcal{S}_{n_{k}}\right)_{k=1}^{\infty}$.

(iv) The spaces obtained by families $\left(\frac{1}{m_{k}}, \mathcal{S}_{n_{k}}\right)_{k=1}^{\infty}$ have the property that every block subspace is an asymptotic $\ell^{1}$-space.

(v) All the mixed Tsirelson $d$-product norms are shrinking. Assuming further that there exists some $k$ such that the Cantor-Bendixson index $i\left(\mathcal{M}_{k}\right)>\theta_{k}^{-1}$, then the norm is boundedly complete. For example the later condition is always satisfied in the AD-constructions and also in Schlumprecht space. In such a case if $\left(X_{n}\right)_{n \in \mathbb{N}}$ are reflexive, then the $d$-product $\tilde{X}$ is also reflexive.

(vi) Let us point out that the finite collections of the form $\left(\frac{1}{m_{i}}, \mathcal{A}_{n_{i}}\right)_{i=1}^{k}$ define norms equivalent to $\left(\sum \oplus X_{n}\right)_{p}$ for some $1<p<\infty$ or $\left(\sum \oplus X_{n}\right)_{0}$ ([B], [AD1] $)$.

We pass now to define block-H.I. $d$-product spaces.

7.2. Gowers-Maurey type block-H.I. spaces. Let $\left(X_{n},\|\|_{n}\right)_{n \in \mathbb{N}}$ be a sequence of separable Banach spaces. Choose $\mathcal{F}_{n}$ to be a countable symmetric subset of $B_{X_{n}^{*}}$ which defines the norm of $X_{n}$ (i.e. $\|x\|=\sup \left\{x^{*}(x): x \in \mathcal{F}_{n}\right\}$ for $\left.x \in X_{n}\right)^{n}$. 
Consider the vector space $\Omega_{00}^{*}=\left(\prod_{n=1}^{\infty} X_{n}^{*}\right)_{00}$ and denote by $\mathcal{K}$ the smallest subset of $\Omega_{00}^{*}$ containing $\bigcup_{n=1}^{\infty} \mathcal{F}_{n}$ and closed under the following operations.

(1) For every $\phi_{1}<\ldots<\phi_{N}$ elements of $\mathcal{K}$ the vectors $\frac{1}{\log _{2}(N+1)} \sum_{i=1}^{N} \phi_{i}$ and $\frac{1}{\sqrt{\log _{2}(N+1)}} \sum_{i=1}^{N} \phi_{i}$ also belong to $\mathcal{K}$.

(2) For every $\phi \in \mathcal{K}$ and $E$ segment of $\mathbb{N}, E \phi \in \mathcal{K}$.

(3) For every $E \in \mathcal{K}$ and $r \in \mathbb{Q} \cap[-1,1], r \phi \in \mathcal{K}$. ( $\mathbb{Q}$ denotes the set of rational numbers.)

The set $\mathcal{K}$ is defined in a similar manner as in Proposition 7.1. Indeed, by induction, we produce an increasing sequence $\left(\mathcal{K}_{l}\right)_{l=0}^{\infty}$ in the following manner.

$$
\begin{aligned}
& \mathcal{K}_{0}=\bigcup_{n=1}^{\infty} \mathcal{F}_{n} \text { and if } \mathcal{K}_{l} \text { has been defined we set } \\
& \mathcal{K}_{l+1}=\mathcal{K}_{l} \cup\left\{\begin{array}{c}
\frac{r}{g(N)} \sum_{i=1}^{N} E \phi_{i}: g(N)=\log _{2}(N) \\
\text { or } g(N)=\sqrt{\log _{2}(N)}, r \in \mathbb{Q} \cap[-1,1], \\
E \subset \mathbb{N} \text { is an interval and } \phi_{1}<\ldots<\phi_{N} \text { belong to } \mathcal{K}_{l}
\end{array}\right\} .
\end{aligned}
$$

It is easily checked that the set $\mathcal{K}=\bigcup_{l=0}^{\infty} \mathcal{K}_{l}$ satisfies the desired properties. Further the following additional properties can be established for the set $\mathcal{K}$.

(4) $\mathcal{K}$ is a countable set.

(5) For every $\phi \in \mathcal{K}$ and $n \in \mathbb{N},\|\phi(n)\|_{n} \leq 1$. Here by $\phi(n)$ we denote the $n^{t h}$ coordinate of $\phi$ which of course belongs to $X_{n}^{*}$.

Next, as in the Gowers-Maurey proof, we will define an injection $\sigma$ from the set $\mathcal{K}^{(\mathbb{N})}=\left\{\left(\phi_{1}, \ldots, \phi_{n}\right): \phi_{1}<\ldots<\phi_{n}, \phi_{i} \in \mathcal{K}\right\}$ to a certain set $L$, a subset of $\mathbb{N}$. We begin by considering a very fast increasing sequence $J=\left(j_{n}\right)_{n=1}^{\infty}$ in $\mathbb{N}$. More precisely we define $J$ such that $\log _{2}\left(j_{1}+1\right)>256$ and for all $n \in \mathbb{N}$, $\log \log \log \left(j_{n+1}\right)>4 j_{n}^{2}$. We denote by $K$ the set $\left\{j_{2 i+1}\right\}_{i=0}^{\infty}$ and by $L$ the set $\left\{j_{2 i}\right\}_{i=1}^{\infty}$. We define an injection $\sigma: \mathcal{K}^{(\mathbb{N})} \rightarrow L$ such that for $\left(\phi_{1}, \ldots, \phi_{N}\right) \in \mathcal{K}^{(\mathbb{N})}$ if $S=\sigma\left(\phi_{1}, \ldots, \phi_{N}\right), \phi=\sum_{i=1}^{N} \phi_{i}$, then $\frac{1}{20} \log _{2}\left(S^{\frac{1}{40}}+1\right) \geq \mid$ range $(\phi) \mid$.

The H.I. norm we wish to define will be obtained by a symmetric subset $\mathcal{D}$ of the set $\mathcal{K}$ defined above. We start with the following two definitions.

Definition 7.2. Let $\mathcal{D}$ be a subset of $\Omega_{00}^{*}$ and let $g:[1, \infty] \rightarrow[1, \infty]$ be a function. A vector $\phi$ in $\mathcal{D}$ is said to be an $(M, g)$-form with respect to $\mathcal{D}$ if there exists $d \leq M$ and a sequence $\left(\phi_{i}\right)_{i=1}^{d}$ of successive blocks such that $\phi=\frac{1}{g(M)} \sum_{i=1}^{d} \phi_{i}$ and $\phi_{i} \in \mathcal{D}$ for $i=1, \ldots, d$.

An easy consequence of the inductive definition of the set $\mathcal{K}$ is that every $\phi \in \mathcal{K}$ is either $\left(M, \log _{2}(x+1)\right)$ or an $\left(M, \sqrt{\log _{2}(x+1)}\right)$-form with respect to $\mathcal{K}$.

Definition 7.3. Let $\mathcal{K}$ and $\sigma: \mathcal{K}^{(\mathbb{N})} \rightarrow L$ be the set and the function defined above. A finite sequence $\left(\phi_{1}, \ldots, \phi_{N}\right) \in \mathcal{K}^{(\mathbb{N})}$ is said to be a special sequence provided that there exists $\left\{M_{1}, \ldots, M_{N}\right\} \subset L$ such that each $\phi_{i}$ is an $\left(M_{i}, \log _{2}(x+1)\right)$-form with respect to $\mathcal{K}$ and for $i=2, \ldots, N, M_{i}=\sigma\left(\phi_{1}, \ldots, \phi_{i-1}\right)$. 
The norming set $\mathcal{D}$. The set $\mathcal{D}$ is inductively defined as follows. We set $\mathcal{D}_{0}=$ $\left\{\phi: \phi=r \phi^{\prime}, r \in[-1,1] \cap \mathbb{Q}, \phi^{\prime} \in \bigcup_{n=1}^{\infty} \mathcal{F}_{n}\right\}$. Assume that $\mathcal{D}_{n}$ has been constructed and we define $\mathcal{D}_{n+1}$ as follows:

We set

$$
\begin{gathered}
\mathcal{E}_{n+1}=\left\{\frac{r}{\log _{2}(N+1)} \sum_{i=1}^{N} \phi_{i}: \phi_{1}<\ldots<\phi_{N}, \phi_{i} \in \mathcal{D}_{n}, r \in \mathbb{Q} \cap[-1,1]\right\}, \\
\mathcal{F}_{n+1}=\left\{\frac{r}{\sqrt{\log _{2}(N+1)}} \sum_{i=1}^{N} \phi_{i}:\left(\phi_{1}, \ldots, \phi_{N}\right)\right. \text { is a special sequence, } \\
N \in K, r \in \mathbb{Q} \cap[-1,1]\}
\end{gathered}
$$

and finally

$$
\mathcal{Q}_{n+1}=\left\{E \phi: E \text { is an interval of } \mathbb{N}, \phi \in \mathcal{E}_{n+1} \cup \mathcal{F}_{n+1}\right\} .
$$

We define $\mathcal{D}_{n+1}=\mathcal{D}_{n} \cup \mathcal{Q}_{n+1}$. The desired set $\mathcal{D}$ is the union of all $\left\{\mathcal{D}_{n}\right\}_{n=0}^{\infty}$.

Remark 7.2. The definitions of the function $\sigma$ and the norming set $\mathcal{D}$ presented here are slightly different from the corresponding ones in the Gowers-Maurey space. The reason we follow this approach is to ensure that every $\phi$ belonging to $\mathcal{D}$ is an $(M, g)$-form with respect the set $\mathcal{D}$, where $g$ is either equal to $\log _{2}(x+1)$ or $\sqrt{\log _{2}(x+1)}$. To see this, first we observe that each $\phi \in \mathcal{D}_{0}$ is a $(1, g)$-form; next suppose that $\phi \in \mathcal{D}_{n+1}$ so $\phi=\frac{r}{g(M)} \sum_{i=1}^{M} E \phi_{i}$ where $\phi_{1}, \ldots, \phi_{M}$ are in $\mathcal{D}_{n}$. But from the inductive definition for all $i=1, \ldots, M$ the functional $r E \phi_{i}$ belongs to $\mathcal{D}_{n}$. Hence $\phi=\frac{1}{g(M)} \sum_{i=1}^{M} \phi_{i}^{\prime}$ with $\phi_{i}^{\prime} \in \mathcal{D}_{n}$. Later we will make use of this observation to define the analysis of the elements of $\mathcal{D}$. It is also clear that $\mathcal{D}$ is a symmetric subset of the set $\mathcal{K}$ defined before. Therefore every $(M, g)$-form with respect to $\mathcal{D}$ is also an $(M, g)$-form with respect to $\mathcal{K}$. In the sequel by the term $(M, g)$-form we will mean an $(M, g)$-form with respect to the set $\mathcal{D}$.

Next for $\tilde{x} \in \Omega_{00}=\left(\prod_{n=1}^{\infty} X_{n}\right)_{00}$ we set $\|\tilde{x}\|_{G M}=\sup \{\phi(\tilde{x}): \phi \in \mathcal{D}\}$ and denote by $\tilde{X}_{G M}$ the completion of $\Omega_{00}$ under \|\|$_{G M}$. It follows from the properties of the norming set $\mathcal{D}$ that $\tilde{X}_{G M}$ is a $d$-product of $\left(X_{n},\|\|_{n}\right)_{n \in \mathbb{N}}$ which is additionally 1-bimonotone. We shall prove that:

Theorem 7.2. The space $\tilde{X}_{G M}$ is a block-H.I. Banach space.

The proof of this theorem is done in several steps. As we have mentioned we will follow the Gowers-Maurey approach.

We begin by recalling Schlumbrecht's class of functions. That is, the class $\mathcal{H}$ of all functions $f:[1, \infty] \rightarrow[1, \infty]$ satisfying the following properties:

(i) $f(1)=1$ and $f(x)<x$ for every $x>1$.

(ii) $f$ is strictly increasing and tends to infinity.

(iii) $\lim _{x \rightarrow \infty} x^{-q} f(x)=0$ for every $q>0$.

(iv) The function $\frac{x}{f(x)}$ is concave and non-decreasing.

(v) $f(x y) \leq f(x) f(y)$ for every $x, y \geq 1$. 
It is easily verified that the function $\log _{2}(x+1)$ belongs to $\mathcal{H}$ and if $f \in \mathcal{H}$ then $\sqrt{f}$ also belongs to $\mathcal{H}$.

Definition 7.4. Let $(\tilde{X},\|\|)$ be a $d$-product of the sequence $\left(X_{n},\|\|_{n}\right)_{n \in \mathbb{N}}$, and let $f \in \mathcal{H}$. We say that $(\tilde{X},\|\|)$ satisfies a lower $f$-estimate if for every $\tilde{x} \in X$ $\|\tilde{x}\| \geq \sup \left\{f(N)^{-1} \sum_{i=1}^{N}\left\|E_{i} \tilde{x}\right\|: N \in \mathbb{N}, E_{1}<\ldots<E_{n}, E_{i}\right.$ is an interval of $\left.\mathbb{N}\right\}$.

Remark 7.3. It follows from the properties of the norming set $\mathcal{D}$ that $\tilde{X}_{G M}$ satisfies a lower $\log _{2}(x+1)$-estimate.

We recall that for $(\tilde{X},\|\|)$ a $d$-product of $\left(X_{n},\|\|_{n}\right)_{n \in \mathbb{N}}$, a block subspace is a subspace generated by a sequence $\left(\tilde{y}_{k}\right)_{k \in \mathbb{N}}$ such that supp $\tilde{y}_{k}<$ supp $\tilde{y}_{k+1}$ (see the notation at the beginning of Section 2). Let $\left(\tilde{x}_{i}\right)_{i=1}^{n}$ be a finite block sequence in $\tilde{X}$. Then the vector $\tilde{x}=\sum_{i=1}^{n} \tilde{x}_{i}$ is an $\ell_{1+}^{n}$ average with constant $C \geq 1$ provided $\|\tilde{x}\|=1$ and $\left\|\tilde{x}_{i}\right\| \leq C n^{-1}$. An $\ell_{1+}^{n}$ vector with constant $C$ is any positive multiple of an $\ell_{1+}^{n}$ average with constant $C$.

Lemma 7.3. Let $f \in \mathcal{H}$ and let $(\tilde{X},\|\|)$ be a d-product of $\left(X_{n},\|\|_{n}\right)_{n \in \mathbb{N}}$ satisfying a lower $f$-estimate. Then for every block subspace $Y$ of $\tilde{X}$ and every $C>1$, $n \in \mathbb{N}$, there exist $\tilde{y}_{1}, \ldots, \tilde{y}_{n}$ successive blocks in $Y$ so that $\tilde{y}=\sum_{i=1}^{n} \tilde{y}_{i}$ is an $\ell_{1+}^{n}$ average with constant $C$.

Proof. Suppose that the result is false and let $Y$ be a block subspace of $X, n \geq 2$ and $C>1$ such that $Y$ does not contain any $\ell_{1^{+}}^{n}$-average with constant $C$. Then $Y$ satisfies the following condition:

If $\tilde{x}_{1}<\ldots<\tilde{x}_{n}, n \in \mathbb{N}$, is any block sequence in $Y$ with $\left\|\tilde{x}_{i}\right\| \leq M$ for each $i$, then $\left\|\tilde{x}_{1}+\ldots+\tilde{x}_{n}\right\| \leq n M C^{-1}$.

Let $\left(\tilde{x}_{i}\right)_{i=1}^{\infty}$ be any normalized block sequence of vectors of $Y$. Using the previous observation we prove by induction that for every $s \in \mathbb{N}$ we have

$$
\left\|\sum_{i=1}^{n^{s}} \tilde{x}_{i}\right\| \leq n^{s} C^{-s}
$$

Since $X$ satisfies a lower $f$-estimate,

$$
\left\|\sum_{i=1}^{n^{s}} \tilde{x}_{i}\right\| \geq f\left(n^{s}\right)^{-1} n^{s}
$$

By (11) and (21) $f\left(n^{s}\right) C^{-s} \geq 1$ for every $s \in \mathbb{N}$. Set $q=\log _{n} C$; then $f\left(n^{s}\right)\left(n^{s}\right)^{-q}$ $\geq 1$ for every $s \in \mathbb{N}$ which contradicts the fact that $\lim _{x \rightarrow \infty} f(x) x^{-q}=0$ for every $q>0$.

The next more technical result also concerning $\ell_{1^{+}}^{n}$ averages will be used in the final constructions. 
Lemma 7.4. Let $(\tilde{X},\|\|)$ satisfy a lower $f$-estimate, $\mathcal{D} \subset B_{X^{*}}$ a norming set and $0<\varepsilon<\frac{1}{4}$. Then for every $n \in \mathbb{N}$ and every block subspace $Y$ of $(\tilde{X},\|\|)$ there exist:

(i) $\tilde{x}=\sum_{i=1}^{n} \tilde{x}_{i} \in Y$, an $\ell_{1+}^{n}$ average with constant $1+\varepsilon$.

(ii) $\tilde{y}^{*} \in \mathcal{D}$ such that $\tilde{y}^{*}(\tilde{x})>1-\varepsilon$ and range $(\tilde{x}) \subset \operatorname{range}\left(\tilde{y}^{*}\right)$.

Proof. For a given $n \in \mathbb{N}$ we select $\varepsilon^{\prime}>0$ such that $\varepsilon^{\prime}<\frac{\varepsilon}{n^{2}}$. Then

$$
\frac{\left(1+\varepsilon^{\prime}\right)(n-1)}{n+2}<\frac{\left(1+\frac{1}{n}\right)(n-1)}{n}=\left(1-\frac{1}{n^{2}}\right)<1-\varepsilon^{\prime} .
$$

From the previous lemma for every block subspace $Y$ of $\tilde{X}$ there exists $\tilde{x}=\sum_{i=1}^{n+2} \tilde{x}_{i}$, an $\ell_{1+}^{n+2}$ average with constant $1+\varepsilon^{\prime}$. We choose $\tilde{x}^{*} \in \mathcal{D}$ such that $\tilde{x}^{*}(\tilde{x})>1-\varepsilon^{\prime}$. We set $\tilde{z}=\sum_{i=2}^{n+1} \tilde{x}_{i}$ and $\tilde{y}=\sum_{i=2}^{n+1} \frac{\tilde{x}_{i}}{\|\tilde{z}\|}=\sum_{i=1}^{n} \tilde{y}_{i}$. Evidently, $1-2 \frac{\varepsilon^{\prime}}{n+2} \leq\|\tilde{z}\| \leq 1$. Using (1) we get that $\tilde{x}^{*}\left(\tilde{x}_{i}\right) \neq 0$ for all $i=1, \ldots, n+2$ and so range $(\tilde{y}) \subset \operatorname{range}\left(\tilde{x}^{*}\right)$. Also, $x^{*}(\tilde{y}) \geq \frac{1-\varepsilon^{\prime}-2 \frac{\varepsilon^{\prime}}{n+2}}{1-2 \frac{\varepsilon^{\prime}}{n+2}}>1-\varepsilon$. Finally, $\left\|\tilde{y}_{i}\right\| \leq \frac{\frac{1+\varepsilon^{\prime}}{n+2}}{1-2 \frac{\varepsilon^{\prime}}{n+2}}=\frac{1+\varepsilon^{\prime}}{n+2-2 \varepsilon^{\prime}} \leq \frac{1+\varepsilon^{\prime}}{n}<\frac{1+\varepsilon}{n}$ and therefore $\tilde{y}$ is an $\ell_{1+}^{n}$ average with constant $1+\varepsilon$.

Lemma 7.5. Let $M, N \in \mathbb{N}$, let $C \geq 1$, let $(\tilde{X},\|\|)$ be a bimonotone d-product and let $\tilde{x} \in \tilde{X}$ be an $\ell_{1+}^{n}$ vector with constant $C$. Also let $E_{1}<\ldots<E_{M}$ be a sequence of intervals. Then

$$
\sum_{j=1}^{M}\left\|E_{j} \tilde{x}\right\| \leq C\left(1+\frac{2 M}{N}\right)\|\tilde{x}\| .
$$

In particular, if $M \leq N$, then $\sum_{j=1}^{M}\left\|E_{j} \tilde{x}\right\| \leq 3 C\|\tilde{x}\|$.

The proof of this lemma is quite easy and we refer the reader to Lemma 4 of GM].

Let $f(x)=\log _{2}(x+1)$ and let $M_{f}: \mathbb{R} \rightarrow \mathbb{R}$ be the function defined by $M_{f}(x)=$ $f^{-1}\left(36 x^{2}\right)$. For $\varepsilon>0$ we denote by $\varepsilon^{\prime}$ the number $\min \{\varepsilon, 1\}$. We shall say that a sequence $\tilde{x}_{1}<\ldots<\tilde{x}_{N}$ is a rapidly increasing sequence (or R.I.S.) of $\ell_{1}+$ averages, for $f$ of length $N$ with constant $1+\varepsilon$, if $x_{k}$ is an $\ell_{1+}^{n_{k}}$ average with constant $1+\varepsilon$ for each $k, n_{1} \geq \frac{2(1+\varepsilon) M_{f}\left(N / \varepsilon^{\prime}\right)}{\varepsilon^{\prime} f^{\prime}(1)}$, and

$$
\frac{\varepsilon^{\prime}}{2} \sqrt{f\left(n_{k}\right)} \geq\left|\operatorname{range}\left(\tilde{x}_{k-1}\right)\right|
$$

for $k=2, \ldots, N$. Here $f^{\prime}(1)$ is the derivative of $f$ at 1 .

Next we give the statements of two lemmas which correspond to Lemmas 10 and 12 in [GM]. Their proofs are exactly the same as those of Lemmas 10 and 12 in GM]. Thus we refer the reader to Gowers-Maurey's paper for the proof of these results. We should point out that Lemmas 10 and 12 in [GM] constitute the hard part of the proof that the space is H.I. In their proofs Gowers and Maurey make elegant use of the properties of Schlumprecht class $\mathcal{H}$. Lemma 10 is of an 
unconditional nature and the important role of special sequences appears in Lemma 12.

Lemma 7.6. Let $N \in L, n \in[\log N, \exp N]$ and $\varepsilon>0$. Also let $\tilde{x}_{1}, \ldots, \tilde{x}_{n}$ be a R.I.S. with constant $1+\varepsilon$. Then $\left\|\sum_{i=1}^{n} \tilde{x}_{i}\right\|_{G M} \leq\left(1+\varepsilon+\varepsilon^{\prime}\right) \frac{n}{\log _{2}(n+1)}$.

Let us observe that since $\tilde{X}_{G M}$ satisfies a lower $\log _{2}(n+1)$-estimate we get that $\frac{n}{\log _{2}(n+1)} \leq\left\|\sum_{i=1}^{n} \tilde{x}_{i}\right\|_{G M}$.

Lemma 7.7. Let $k \in K$ and let $\tilde{x}_{1}^{*}, \ldots, \tilde{x}_{k}^{*}$ be a special sequence of length $k$, where each $\tilde{x}_{i}^{*}$ is an $\left(M_{i}, \log _{2}(x+1)\right)$-form. Let $\tilde{x}_{1}, \ldots, \tilde{x}_{k}$ be a sequence of successive vectors such that every $\tilde{x}_{i}$ is a normalized R.I.S. vector of length $M_{i}$ with constant $1+\frac{\varepsilon}{4}, \varepsilon=\frac{1}{10}$. Assume that range $\left(\tilde{x}_{i}\right) \subset \operatorname{range}\left(\tilde{x}_{i}^{*}\right)$ and $\left|\left(\sum_{i=1}^{k} \tilde{x}_{i}^{*}\right)\left(\sum_{i=1}^{k} E \tilde{x}_{i}\right)\right| \leq 2$ for every interval $E$. Then

$$
\left\|\sum_{i=1}^{k} \tilde{x}_{i}\right\|_{G M} \leq(1+2 \varepsilon) \frac{k}{\log _{2}(k+1)} .
$$

Let us notice that the statement of Lemma 7.7 is slightly different from the corresponding statement in Lemma 12 in [GM]. In spite of this change the proof of this lemma goes exactly along the lines of the proof of Lemma 12 in [GM].

Completion of the proof of Theorem 7.2 Let $Y$ and $Z$ be block subspaces of $\left(\tilde{X}_{G M},\|\|_{G M}\right)$. For $\varepsilon=\frac{1}{10}, k \in K$ we choose $M_{1}=j_{2 k} \in L$ such that $M_{1}^{\frac{\varepsilon}{4}} \geq$ $N_{1} \geq \frac{4 M_{f}\left(\frac{k}{\varepsilon}\right)}{\varepsilon f^{\prime}(1)}$ where $f(x)=\log _{2}(x+1)$. We inductively define $\tilde{x}_{1}, \ldots, \tilde{x}_{k}, \tilde{x}_{1}^{*}, \ldots, \tilde{x}_{k}^{*}$ so that the following conditions are fulfilled:

- $\tilde{x}_{2 j+1} \in Y, \tilde{x}_{2 j} \in Z$ and $\tilde{x}_{1}<\ldots<\tilde{x}_{k}$.

- $\tilde{x}_{i}^{*}$ is an $\left(M_{i}, f\right)$-form with respect to the set $\mathcal{D}$, range $\left(\tilde{x}_{i}\right) \subset \operatorname{range}\left(\tilde{x}_{i}^{*}\right)$ and $\left(\tilde{x}_{1}^{*}, \ldots, \tilde{x}_{k}^{*}\right)$ is a special sequence.

- Each $\tilde{x}_{i}$ is a normalized R.I.S. of length $M_{i}$ and $\left|\tilde{x}_{i}^{*}\left(\tilde{x}_{i}\right)-\frac{1}{2}\right|<\frac{1}{k}$.

To prove that such a choice is possible we follow closely the corresponding construction in [GM], p.868. We begin with the definitions of $\tilde{x}_{1}$ and $\tilde{x}_{1}^{*}$. We first choose $\tilde{x}_{11}, \ldots, \tilde{x}_{1 M_{1}}$ a R.I.S. of $\ell_{1}+$ averages with constant $1+\frac{\varepsilon}{4}$. For each $\tilde{x}_{1 j}$ we select $\tilde{x}_{1 j}^{*}$ in $\mathcal{D}$ such that $\tilde{x}_{1 j}^{*}\left(\tilde{x}_{1 j}\right)>1-\frac{\varepsilon}{4}$ and further assume that range $\left(\tilde{x}_{1 j}\right) \subset \operatorname{range}\left(\tilde{x}_{1 j}^{*}\right)$ (Lemma 7.3).

According to Lemma 7.6 we have that

$$
\left\|\sum_{j=1}^{M_{1}} \tilde{x}_{1 j}\right\|_{G M} \leq\left(1+\frac{\varepsilon}{2}\right) M_{1} \log _{2}^{-1}\left(M_{1}+1\right)
$$

and also $\frac{1}{\log _{2}\left(M_{1}+1\right)} \sum_{j=1}^{M_{1}} \tilde{x}_{1 j}^{*}\left(\sum_{j=1}^{M_{1}} \tilde{x}_{1 j}\right) \geq\left(1-\frac{\varepsilon}{4}\right) \frac{M_{1}}{\log _{2}\left(M_{1}+1\right)}$. 
Therefore setting $\tilde{x}_{1}$ to be the normalized multiple of $\sum_{j=1}^{M_{1}} \tilde{x}_{1 j}$ we get that $\frac{1}{\log _{2}\left(M_{1}+1\right)} \sum_{j=1}^{M_{1}} \tilde{x}_{1 j}^{*}\left(\tilde{x}_{1}\right)>\frac{1}{2}$ and so there exists $r \in[0,1] \cap \mathbb{Q}$ such that

$$
\left|\frac{r}{\log _{2}\left(M_{1}+1\right)} \sum_{j=1}^{M_{1}} \tilde{x}_{1 j}^{*}\left(\tilde{x}_{1}\right)-\frac{1}{2}\right|<\frac{1}{k} .
$$

We set $\tilde{x}_{1}^{*}=\frac{r}{\log _{2}\left(M_{1}+1\right)} \sum_{j=1}^{M_{1}} \tilde{x}_{1 j}^{*}$ which clearly belongs to $\mathcal{D}$ and further $\operatorname{range}\left(\tilde{x}_{1}\right) \subset$ range $\left(\tilde{x}_{1}^{*}\right)$. Thus the choice of $\tilde{x}_{1}, \tilde{x}_{1}^{*}$ is complete. To choose $\tilde{x}_{2}, \tilde{x}_{2}^{*}$ we set $M_{2}=$ $\sigma\left(\tilde{x}_{1}^{*}\right)$ and we repeat the above procedure for the number $M_{2}$ instead of $M_{1}$ and $\tilde{x}_{2 j} \in Z, j=1, \ldots, M_{2}$, such that $\tilde{x}_{1}<\tilde{x}_{21}<\ldots<\tilde{x}_{2 M_{2}}$. In the general inductive step we proceed in a similar manner. It remains to prove that $Y+Z$ does not form a topological direct sum. We define $\tilde{y}=\tilde{x}_{1}+\tilde{x}_{3}+\ldots$ and $\tilde{z}=\tilde{x}_{2}+\tilde{x}_{4}+\ldots$. Then $\tilde{y} \in Y$ and $\tilde{z} \in Z$. Further $\|\tilde{y}+\tilde{z}\|_{G M}=\left\|\sum_{i=1}^{k} \tilde{x}_{i}\right\|_{G M} \geq \frac{1}{\sqrt{\log _{2}(k+1)}} \sum_{i=1}^{k} \tilde{x}_{i}^{*}\left(\tilde{x}_{i}\right) \geq$ $\frac{1}{\sqrt{\log _{2}(k+1)}}\left(\frac{k}{2}-1\right)$. Also since $\left((-1)^{i-1} \tilde{x}_{i}\right)_{i=1}^{k},\left(\tilde{x}_{i}^{*}\right)_{i=1}^{k}$ satisfy the assumptions of Lemma 7.7 we have that $\|\tilde{y}-\tilde{z}\|_{G M}=\left\|\sum_{i=1}^{k}(-1)^{i-1} \tilde{x}_{i}\right\|_{G M} \leq(1+2 \varepsilon) k \frac{1}{\log _{2}(k+1)}$.

Hence for every $\delta>0$ there exist $\tilde{y} \in Y, \tilde{z} \in Z$ such that $\|\tilde{y}+\tilde{z}\|_{G M} \geq$ $\delta^{-1}\|\tilde{y}-\tilde{z}\|_{G M}$ which immediately shows that the sum $Y+Z$ is not closed. The proof of the theorem is complete.

Proposition 7.8. For every sequence $\left(X_{n}\right)_{n \in \mathbb{N}}$ of separable Banach spaces the $d$ product space $\tilde{X}_{G M}$ defined above is boundedly complete and shrinking. Therefore if each $X_{n}$ is a reflexive space, then the space $\tilde{X}_{G M}$ is also reflexive.

(For a proof we refer to p. 869 of [GM].)

Remark 7.4. It is also possible to define block-H.I. spaces with an adaptation of the construction of asymptotic $\ell^{1}$ H.I. spaces contained in AD2. Such a construction will produce block-H.I. spaces with the additional property that every block subspace is an asymptotic $\ell^{1}$ space.

The rest of this section is devoted to some results which will be used to show that there exist H.I. spaces $X$ and $T: X \rightarrow X$ which is a strictly singular and noncompact operator. For the proof of these results we use the analysis of a functional $\phi \in \mathcal{D}$ introduced in $\mathrm{AD2}$ and defined below.

Definition 7.5. Let $\phi \in \mathcal{D}$. An analysis of $\phi$ is a sequence $\left(\mathcal{D}_{s}(\phi)\right)_{s=0}^{m}$ such that

(1) $\mathcal{D}_{s}(\phi)=\left\{f_{1}, \ldots, f_{d_{s}}\right\}$ where $f_{1}<\ldots<f_{d_{s}}$ and $f_{i} \in \mathcal{D}_{s}$ for $i=1, \ldots, d_{s}$,

(2) $\bigcup_{\left.f \in \mathcal{D}_{s}(\phi)\right)} \operatorname{suppf}=\operatorname{supp} \phi$,

(3) if $s>0$ and $\psi \in K^{s}(\phi) \backslash K^{s-1}(\phi)$, then there exist $M \in \mathbb{N}$ and $\psi_{1}, \ldots, \psi_{d}$, $d \leq M$, elements of $\mathcal{D}_{s-1}(\phi)$ such that $\psi=\frac{1}{g(M)} \sum_{i=1}^{d} \psi_{i}$ where $g(M)=\log _{2}(M+1)$ or $g(M)=\sqrt{\log _{2}(M+1)}$ and

(4) $\mathcal{D}_{m}(\phi)=\{\phi\}$. 
Let us observe that every $\phi \in \mathcal{D}$ has an analysis $\left(\mathcal{D}_{s}\right)_{s=0}^{m}$ (see Remark 7.2$)$.

Definition 7.6. A sequence $\left(\tilde{x}_{i}\right)_{i=1}^{\infty}$ of successive blocks of $\tilde{X}_{G M}$ is called an infinite rapidly increasing sequence or a R.I.S. of constant $C$ if $\tilde{x}_{i}$ is an $\ell_{1^{+}}^{n_{i}}$ average of constant $C$ and for each $i \in \mathbb{N}$ it holds that $\sqrt{\log _{2}\left(n_{i+1}\right)}>2^{i}\left|\operatorname{range}\left(\tilde{x}_{i}\right)\right|$.

In the sequel by $S_{3}$ we denote the mixed Tsirelson space $T\left(\frac{1}{\sqrt{\log _{2}(n+1)}}, \mathcal{A}_{3 n}\right)_{n \in \mathbb{N}}$ and by $\mathcal{K}_{S_{3}}$ the norming set of $S_{3}$ as it is defined in Definition 7.1 and Remark 7.1 $(i)$.

Proposition 7.9. Let $\left(\tilde{x}_{i}\right)_{i=1}^{\infty}$ be a R.I.S. in $\tilde{X}_{G M}$ of $\ell_{1+}^{n_{i}}$ average with constant $C<\frac{4}{3}$. Then for every $\phi \in K$ there exists $\phi^{\prime} \in K_{S_{3}}$ such that for every $\left(\lambda_{i}\right)_{i=1}^{\infty} \subset \mathbb{R}$, $\left|\phi\left(\sum_{i=1}^{\infty} \lambda_{i} \tilde{x}_{i}\right)\right| \leq 4 \phi^{\prime}\left(\sum_{i=1}^{\infty}\left|\lambda_{i}\right| e_{i}\right)+2 \max \left\{\left|\lambda_{i}\right|: i \in \mathbb{N}\right\}$. Therefore $\left\|\sum_{i=1}^{\infty} \lambda_{i} \tilde{x}_{i}\right\|_{G M} \leq$ $6\left\|\sum_{i=1}^{\infty} \lambda_{i} e_{i}\right\|_{S_{3}}$.

Proof. For $\phi \in \mathcal{D}$, we choose an analysis $\left(\mathcal{D}_{s}(\phi)\right)_{s=0}^{m}$ of $\phi$. Let $I=\left\{i \in \mathbb{N}: \operatorname{supp}\left(\tilde{x}_{i}\right)\right.$ $\cap \operatorname{supp}(\phi) \neq \emptyset\}$. For each $i \in I$ there exist a unique element $\phi_{i} \in \bigcup_{s=0}^{m} \mathcal{D}_{s}(\phi)$ and an element $s_{i} \in\{0, \ldots, m\}$ satisfying the following two conditions:

(1) $\operatorname{supp}_{i} \cap$ supp $\tilde{x}_{i}=\operatorname{supp} \phi \cap \operatorname{supp} \tilde{x}_{i}$ and

(2) $\phi_{i} \in \mathcal{K}^{s_{i}}(\phi)$ and for $s<s_{i}$ there is no $\psi \in \mathcal{K}^{s}(\phi)$ satisfying (1) for the vector $\tilde{x}_{i}$.

For every $i \in I$ such that $s_{i}>0$ let $M_{i}$ be the natural number such that $\phi_{i}=$ $\frac{1}{g\left(M_{i}\right)} \sum_{j=1}^{d_{i}} \psi_{j}$, where $d_{i} \leq M_{i}$ and $\psi_{1}, \ldots, \psi_{d_{i}}$ are successive elements of $\mathcal{D}_{s_{i}-1}(\phi)$.

We set $I^{\prime}=\left\{i \in I: M_{i} \geq n_{i+1}\right\}$ and $I^{\prime \prime}=I \backslash I^{\prime}$.

Observe that for every $i \in I^{\prime},\left|\phi\left(\tilde{x}_{i}\right)\right| \leq \frac{\left|\operatorname{range}\left(\tilde{x}_{i}\right)\right|}{\sqrt{\log _{2}\left(n_{i}+1\right)}}<\frac{1}{2^{i}}$ hence $\left|\phi\left(\sum_{i \in I^{\prime}} \lambda_{i} \tilde{x}_{i}\right)\right|<$ $\max \left\{\left|\lambda_{i}\right|: i \in \mathbb{N}\right\}$.

It remains to estimate $\left|\phi\left(\sum_{i \in I^{\prime \prime}} \lambda_{i} \tilde{x}_{i}\right)\right|$. For every $\psi \in \bigcup_{s=0}^{m} \mathcal{D}_{s}(\phi)$ we set $D_{\psi}=$ $\left\{i \in I^{\prime \prime}:\right.$ supp $\psi \cap$ supp $\left.\tilde{x}_{i}=\operatorname{supp} \phi \cap \operatorname{supp} \tilde{x}_{i}\right\}$.

For every $\psi \in \bigcup_{s=0}^{m} \mathcal{D}_{s}(\phi)$ we inductively define a functional $\psi^{\prime} \in 2 \mathcal{K}_{S_{3}}$ such that the following are fulfilled:

(a) $\operatorname{supp} \psi^{\prime} \subset D_{\psi}$,

(b) $\left|\psi\left(\tilde{x}_{i}\right)\right| \leq 4 \psi^{\prime}\left(e_{i}\right)$ and

(c) one of the following two alternatives holds:

$\left(\mathrm{c}_{1}\right) \psi^{\prime} \in \mathcal{K}_{S_{3}}$ or

$\left(\mathrm{c}_{2}\right)$ there exists $\psi^{(1)} \in \mathcal{K}_{S_{3}}$ and $i \in I^{\prime \prime}, i<\operatorname{supp} \psi^{(1)}$ such that $\psi^{\prime}=e_{i}^{*}+\psi^{(1)}$.

For $s=0$ the construction is obvious. Suppose that for all $\psi \in \bigcup_{t=0}^{s-1} \mathcal{D}_{t}(\phi)$, $\psi^{\prime}$ has been defined and let $\psi \in \mathcal{D}_{s}(\phi) \backslash \mathcal{D}_{s-1}, \psi=\frac{1}{g(M)}\left(\psi_{1}+\ldots+\psi_{d}\right)$ where $\psi_{1}<\ldots<\psi_{d}$ are successive elements of $\mathcal{K}^{s-1}(\phi)$ and $g(M)=\log _{2}(M+1)$ or $g(M)=\sqrt{\log _{2}(M+1)}$. We set $J=D_{\psi} \backslash \bigcup_{j=1}^{M} D_{\psi_{j}}$. 
Case 1. For every $i \in J, M \notin\left(n_{i}, n_{i+1}\right]$.

In this case we define

$$
\psi^{\prime}=\frac{1}{\sqrt{\log _{2}(M+1)}}\left(\sum_{j=1}^{d} \psi_{j}^{\prime}+\sum_{i \in J} e_{i}^{*}\right) .
$$

Evidently condition (a) is fulfilled. To show that condition $\left(\mathrm{c}_{1}\right)$ also holds we set $F_{1}=\left\{j \in\{1, \ldots, d\}: \psi^{\prime} \in \mathcal{K}_{S_{3}}\right\}$ and $F_{2}=\{1, \ldots, d\} \backslash F_{1}$.

For every $j \in F_{2}, \psi_{j}^{\prime}=\psi_{j}^{(1)}+e_{i_{j}}^{*}$ where $i_{j}<\min \operatorname{supp}\left(\psi_{j}^{(1)}\right)$. So $\psi^{\prime}=$ $\frac{1}{\sqrt{\log _{2}(M+1)}}\left[\sum_{j \in F_{1}} \psi_{j}^{\prime}+\sum_{j \in F_{2}} \psi_{j}^{(1)}+\sum_{j \in F_{2}} e_{i_{j}}^{*}+\sum_{i \in J} e_{i}^{*}\right]$.

Since $\left|F_{1}\right|+\left|F_{2}\right|+|J| \leq 2 M$ and $\left|F_{2}\right| \leq M$ it follows that $\psi^{\prime} \in \mathcal{K}_{S_{3}}$. It remains to show that condition (b) holds. Indeed if $i \notin J$, then there exists a $j \in\{1, \ldots, d\}$ such that supp $_{j} \cap \operatorname{supp} \tilde{x}_{i}=\operatorname{supp} \phi \cap \operatorname{supp} \tilde{x}_{i}$. By the inductive hypothesis $\left|\psi\left(\tilde{x}_{i}\right)\right|=$ $\frac{1}{g(M)}\left|\psi_{j}\left(\tilde{x}_{i}\right)\right| \leq \frac{1}{\sqrt{\log _{2}(M+1)}}\left|\psi_{j}\left(\tilde{x}_{i}\right)\right| \leq \frac{4}{\sqrt{\log _{2}(M+1)}} \psi_{j}^{\prime}\left(e_{i}\right)=4 \psi^{\prime}\left(e_{i}\right)$. If $i \in J$, then $\psi=\phi_{i}$ and so $M \leq n_{i+1}$. But $M \notin\left(n_{i}, n_{i+1}\right]$ and therefore $M \leq n_{i}$. From Lemma 7.5 we get that $\left|\psi\left(\tilde{x}_{i}\right)\right| \leq \frac{1}{g(M)} C\left(1+\frac{2 M}{n_{i}}\right)<\frac{4}{\sqrt{\log _{2}(M+1)}}=4 \psi^{\prime}\left(e_{i}\right)$.

Case 2. There exists (a unique) $i_{0} \in J$ such that $n_{i_{0}}<M \leq n_{i_{0}+1}$.

In this case we define

$$
\psi^{(1)}=\frac{1}{\sqrt{\log _{2}(M+1)}}\left(\sum_{j=1}^{d} \psi_{j}^{\prime}+\sum_{i \in J \backslash\left\{i_{0}\right\}} e_{i}^{*}\right), \quad \psi^{\prime}=\psi^{(1)}+e_{i_{0}}^{*} .
$$

Arguing as in Case 1 one can show that $\psi^{\prime}$ satisfies conditions (a), (b) and $\left(\mathrm{c}_{2}\right)$ of the inductive hypothesis. Thus the inductive definition is complete.

It follows now that there exists $\phi^{\prime}$ such that either $\phi^{\prime} \in \mathcal{K}_{S_{3}}$ and $\left|\phi\left(\tilde{x}_{i}\right)\right| \leq 4 \phi^{\prime}\left(e_{i}\right)$ for each $i \in I^{\prime \prime}$ or $\phi^{\prime}=e_{i_{0}}^{*}+\phi^{(1)}$ and $\phi\left(\tilde{x}_{i}\right) \leq 4 \phi^{(1)}\left(e_{i}\right)$ for any $i \in I^{\prime \prime} \backslash\left\{i_{0}\right\}$. The desired result follows easily from this and the proof is complete.

As a consequence of the above proposition we have the following:

Corollary 7.10. Let $\left(\tilde{x}_{i}\right)_{i=1}^{\infty}$ be an infinite R.I.S. in $\tilde{X}_{G M}, Z=\left\langle\left(\tilde{x}_{i}\right)_{i=1}^{\infty}\right\rangle$ and $T: S_{3} \rightarrow Z$ the linear operator defined by $T\left(e_{i}\right)=\tilde{x}_{i}$ for every $i \in \mathbb{N}$. Then $T$ is a bounded non-compact operator.

In the last part of this section we will show that the space $S_{3}$ satisfies property $\left(P_{2}\right)$ (see Remark 4.1). Let us observe that $S_{3}$ satisfies a lower $g$ estimate where $g(x)=\log _{2}\left(\frac{x}{3}+1\right)$ for $x \geq 3$. Therefore from Lemma 7.3 every block subspace $Y$ of $S_{3}$ contains $\ell_{1+}^{n}$ averages with constant $C$ for all $n \geq 3$ and $C>1$. Also it is obvious that $S_{3}$ is a mixed Tsirelson space with an unconditional basis and hence every $\phi \in \mathcal{K}_{S_{3}}$ admits an analysis. The definitions of $\ell_{1+}^{n}$ averages with constant $C$ and the infinite R.I.S. in block subspaces of $S_{3}$ are exactly the same as in the space $\tilde{X}_{G M}$. Finally we denote by $S_{9}$ the space $T\left[\left(\frac{1}{\sqrt{\log _{2}(N+1)}}, \mathcal{A}_{9 N}\right)_{N=1}^{\infty}\right]$.

Lemma 7.11. Let $Y$ be a block subspace of $S_{3}$ and let $\left(x_{i}\right)_{i=1}^{\infty}$ be an infinite R.I.S. in $Y$ with constant $1+\varepsilon, 0<\varepsilon<1$. Then for every $\left(\lambda_{i}\right)_{i=1}^{\infty} \subset \mathbb{R}$ with $\left|\lambda_{i}\right| \leq 1$, $\left\|\sum_{i=1}^{\infty} \lambda_{i} x_{i}\right\|_{S_{3}} \leq 6\left\|\sum_{i=1}^{\infty} \lambda_{i} x_{i}\right\|_{S_{9}}$ 
The proof of this lemma is similar to that of Proposition 7.9. In fact we may prove that for every $\phi \in \mathcal{K}_{S_{3}}$ there exists a $\phi^{\prime} \in \mathcal{K}_{S_{9}}$ such that $\left|\phi\left(\sum_{i=1}^{\infty} x_{i}\right)\right| \leq$ $4 \phi^{\prime}\left(\sum_{i=1}^{\infty}\left|\lambda_{i}\right| e_{i}\right)+2 \max \left\{\left|\lambda_{i}\right|: i \in \mathbb{N}\right\}$. This can be accomplished by using the analysis of $\phi$. Next we notice that for every $n \in \mathbb{N}$

$$
\left\|\sum_{i=1}^{9 n} e_{i}\right\|_{S_{9}}=\frac{9 n}{\sqrt{\log _{2}(n+1)}} .
$$

Therefore for every infinite R.I.S. $\left(x_{i}\right)_{i=1}^{\infty}$ in the space $S_{3}$ with constant $C<\frac{4}{3}$ we obtain via Lemma 7.11 and (1), that for every $k \in \mathbb{N}$ there exists $n_{k}$ such that for $i_{1}<\ldots<i_{n_{k}}$,

$$
\frac{\left\|x_{i_{1}}+\ldots+x_{i_{n_{k}}}\right\|_{S_{3}}}{n_{k}}<\frac{6}{\sqrt{\log _{2}\left(n_{k}+1\right)}}<\frac{1}{k}
$$

Thus we have the following

Corollary 7.12. The space $S_{3}$ satisfies property $\left(P_{2}\right)$. Hence the set $W$ in the space $X_{S_{3}}$ is an a-thin set.

\section{Final Results}

This final section contains the main results of this paper. These are theorems concerning quotients of H.I. spaces and factorization of a-thin operators through H.I. spaces. In the final part we prove similar results for quotients of $\ell^{p}$-saturated Banach spaces.

Theorem 8.1. Let $A$ be a reflexive Banach space with an unconditional basis satisfying property $(P)$. Then there exists a reflexive H.I. Banach space $X$ such that $A$ is a quotient of $X$.

Proof. Recall that property $(P)$ is defined in Definition 4.1. By Theorem 4.9 it follows that the set $W$ in $X_{A}$ is an a-thin set for an appropriate positive null sequence $\mathbf{a}=\left(a_{n}\right)_{n \in \mathbb{N}}$ and it also norms a subspace $Y$ of $X_{A}^{*}$ isometric to $A^{*}$. If \|\|$_{n}$ is the equivalent norm defined by Minkowski's gauge $2^{n} W+a_{n} B_{X_{A}}$, then by Proposition 7.8 the $d$-product $\tilde{X}_{G M}$ of the sequence $\left(\left(X_{A},\|\|_{n}\right)\right)_{n \in \mathbb{N}}$ is reflexive. Since $W$ is an a-thin subset of $X_{A}$ it follows by Theorem 7.2 and Proposition [3.1] that the diagonal space $\Delta \tilde{X}_{G M}$ is a H.I. space. The space $\Delta \tilde{X}_{G M}$ as a subspace of a reflexive space is in itself reflexive and further, $A^{*}$ is isomorphic to a subspace of $\Delta \tilde{X}_{G M}^{*}$ (Proposition 3.4). It follows that $\Delta \tilde{X}_{G M}$ is the desired space and the proof is complete

Theorem 8.2. Suppose that $A$ is either $c_{0}$ or a reflexive space with an unconditional basis such that every block subspace $B$ of $A$ contains a further block subspace $Z$ complemented in $A$. Then $A$ is a quotient of a H.I. space $X$. In the later case $X$ can be chosen to be reflexive.

Proof. The proof is completely analogous to that of Theorem 8.1 We consider the space $X_{A}$ and the set $W$ defined in Section 6 (Theorem 6.1) if $A=c_{0}(\mathbb{N})$ and Section 4 (Theorem 4.11) if the space $A$ is a reflexive space. In either case $W$ is a 
thin norming subset of $X_{A}$ and the argument given in the last part of Theorem 8.1 is still carried over yielding the assertion of Theorem 8.2 .

Next, we list some classical spaces that are quotients of a H.I. space.

Corollary 8.3. Every $L^{p}(\lambda), 1<p<\infty$, $\ell^{p}(\mathbb{N}), 1<p<\infty$, is a quotient of a reflexive H.I. space. Further on, Tsirelson's space $T$ together with its dual and Schlumprecht's space $S$ are quotients of reflexive H.I. spaces.

Proof. It was shown in Section 4 (Remark 4.1 Remark 4.6) that $L^{p}(\lambda), 1<p<$ $\infty, \ell^{p}(\mathbb{N}), 1<p<\infty$, satisfy property $(P)$ and as is well known, they have an unconditional basis. Therefore the result follows from Theorem 8.1

For Tsirelson space $T$, its dual and Schlumprecht space $S$ it is known that they satisfy the complemented subspace condition of Theorem 8.2 Hence, there exist the reflexive H.I. spaces which have them as quotient.

Remark 8.1. (i) It follows by standard duality arguments that if $Y$ denotes one of the spaces $L^{p}(\lambda), 1<p<\infty, \ell^{p}(\mathbb{N}), 1 \leq p<\infty, T, T^{*}, S, S^{*}$, then $Y$ is isomorphic to a subspace of $X^{*}$ for an appropriate H.I. space $X$.

In particular, the embedding of $\ell^{1}(\mathbb{N})$ into $X^{*}$ shows that the dual of a H.I. space is not necessarily arbitrarily distortable. Recall that in [To it has been shown that every H.I. space is arbitrarily distortable.

(ii) As we have already mentioned in the introduction, it is not possible for $\ell^{1}(\mathbb{N})$ to be a quotient of a H.I. space. What's more, it is not possible for $c_{0}(\mathbb{N}), L^{1}(\lambda)$ to be isomorphic to a subspace of $X^{*}$ for $X$ a H.I. space. Indeed, it is well known that if $X^{*}$ contains some of $c_{0}(\mathbb{N}), L^{1}(\lambda)$, then $\ell^{1}(\mathbb{N})$ is isomorphic to a subspace of $X$ which is impossible if $X$ is a H.I. space.

We pass now to prove the dichotomy that we mentioned in the introduction concerning the quotients of H.I. spaces.

Theorem 8.4. Let $A$ be a Banach space. Then either $\ell^{1}(\mathbb{N})$ is isomorphic to a subspace of $A$ or there exists an infinite-dimensional closed subspace $B$ of $A$ which is a quotient of a H.I. space.

Proof. Suppose that $\ell^{1}(\mathbb{N})$ is not isomorphic to a subspace of $A$. Then by Gowers' Dichotomy Theorem ([G]) there exists a subspace $Z$ of $A$ which is either a H.I. space or it has an unconditional basis. If the first case occurs, then we are done. In the second case, by James Theorem ( $\underline{J})$, either $Z$ is a reflexive space or it contains a subspace isomorphic to $c_{0}(\mathbb{N})$. If $c_{0}(\mathbb{N})$ is a subspace of $A$, then as we have shown in Theorem $8.2 c_{0}(\mathbb{N})$ is a quotient of a H.I. space and therefore we get the desired result. The remaining case is that of the space $Z$ being reflexive. It follows from Theorem 5.21 that $Z$ has a subspace $B$ such that the set $W$ in the space $X_{B}$ is an a-thin subset of $X_{B}$ and norming for a subspace of $X_{B}^{*}$ isometric to $B^{*}$. As we have seen before (Theorem 8.1) this implies that $B$ is a quotient of a H.I. space so the proof is complete.

The next result concerns the factorization of linear operators between Banach spaces through H.I. spaces. We recall that a bounded linear operator $T: X \rightarrow Y$ is a-thin, for a positive null sequence $\mathbf{a}=\left(a_{n}\right)_{n \in \mathbb{N}}$, if $T\left[B_{X}\right]$ is an a-thin subset of $Y$.

Theorem 8.5. If $X, Y$ are Banach spaces and $T: X \rightarrow Y$ is an a-thin operator, then there exists a H.I. space $D$ such that $T$ is factorized through $D$. 
Proof. Set $W=T\left[B_{X}\right]$, which by our assumption is an a-thin subset of $Y$. Denote by \|\|$_{n}$ the equivalent norm on $Y$ defined by the Minkowski gauge of the set $2^{n} W+a_{n} B_{Y}$. Consider next the $d$-product $\tilde{X}_{G M}$ of the sequence $\left(\left(Y,\|\|_{n}\right)\right)_{n \in \mathbb{N}}$ which is by Theorem 7.2 a block-H.I. space. The diagonal space $\Delta \tilde{X}_{G M}$ of $\tilde{X}_{G M}$ is a H.I. space (Proposition 3.1) and further on, the unit ball of $\Delta \tilde{X}_{G M}$ contains the set $W$. Therefore the operator $Q: X \rightarrow \Delta \tilde{X}_{G M}$, defined by $Q(x)=T(x)$, is a bounded linear operator and $T=j \circ Q$, where $j: \Delta \tilde{X}_{G M} \rightarrow Y$, is the natural inclusion map. Setting $D=\Delta \tilde{X}_{G M}$ the result follows.

Theorem 8.6. (a) For every $r \neq p, 1 \leq r, p<\infty$, and $T \in \mathcal{L}\left(\ell^{r}, \ell^{p}\right)$, $T$ is factorized through a H.I. Banach space. The same remains valid if one of $\ell^{r}(\mathbb{N})$, $\ell^{p}(\mathbb{N})$ is substituted by $c_{0}(\mathbb{N})$.

(b) Every strictly singular operator $T \in \mathcal{L}\left(\ell^{r}(\mathbb{N}), \ell^{p}(\mathbb{N})\right)$ is factorized through a H.I. space.

(c) The identity map $I: L^{\infty}(\lambda) \rightarrow L^{1}(\lambda)$ is factorized through a H.I. space.

Proof. (a) and (b) follow from Theorem 8.5 and Corollary 4.14. (c) follows from the above Theorem and Proposition 3.3 which assert that $B_{L^{\infty}}$ is an a-thin subset of $L^{1}(\lambda)$.

Next we present a result related to the structure of $\mathcal{L}(X, X)$ for $X$ a H.I. space. In particular we show that there exist H.I. spaces $X$ with "many" $T: X \rightarrow X$ which are strictly singular and not compact.

Theorem 8.7. There exists a H.I. space $X$ such that for every infinite-dimensional closed subspace $Z$ of $X$ there exists a strictly singular operator $T: X \rightarrow Z$ which is not compact.

Proof. We recall that $S_{3}$ denotes the space $T\left[\left(\frac{1}{\sqrt{\log _{2}(n+1)}}, \mathcal{A}_{3 n}\right)_{n=1}^{\infty}\right]$. Then by Corollary 7.12 the set $W$ in $X_{S_{3}}$ is an a-thin set and hence the diagonal space $\Delta \tilde{X}_{G M}$ defined by the set $W$ is a H.I. space that has the space $S_{3}$ as a quotient. Let $Z$ be any closed subspace of $\Delta \tilde{X}_{G M}$; then there exists a normalized basic sequence in $Z$ equivalent to a block sequence $\left(\tilde{y}_{i}\right)_{i=1}^{\infty}$ in $\tilde{X}_{G M}$. It follows (Corollary 7.10) that there exists a non-compact bounded operator $U: S_{3} \rightarrow Z$. If $Q$ denotes the surjection of $\Delta \tilde{X}_{G M}$ onto $S_{3}$, then $U \circ Q$ is a non-compact strictly singular operator from $\Delta \tilde{X}_{G M}$ to $Z$ and the proof is complete.

A by-product of our method is the following result concerning $\ell_{p}(\mathbb{N})(1<p<\infty)$ and $c_{0}(\mathbb{N})$-saturated Banach spaces. First we recall their definition.

Definition 8.1. A Banach space $X$ is said to be $\ell_{p}(\mathbb{N})$-saturated $\left(c_{0}(\mathbb{N})\right.$ saturated) if every subspace $Y$ of $X$ contains a further subspace isomorphic to $\ell_{p}(\mathbb{N})\left(c_{0}(\mathbb{N})\right)$.

Theorem 8.8. Let $A$ be a reflexive Banach space with an unconditional basis. Then there exists a subspace $B$ of $A$ such that for every $p \in(1, \infty)$ there exists an $\ell_{p}(\mathbb{N})$-saturated reflexive space $X_{p}$ which has the space $B$ as a quotient. Further there exists a $c_{0}(\mathbb{N})$-saturated space with the same property.

Proof. According to Theorem 5.21 there exists $B$, a subspace of $A$, with an unconditional basis such that the closed bounded symmetric set $W$ in $X_{B}$ is an a-thin set, norming a subspace $Y$ of $X_{A}^{*}$ isometric to $B^{*}$. We denote by \|\|$_{n}$ the 
equivalent norm defined on $X_{A}$ by the gauge $2^{n} W+a_{n} B_{X_{A}}$ and by $X_{n}$ the space $\left(X,\|\|_{n}\right)$. Then each $X_{n}$ is a reflexive space and the same holds for the space $\tilde{X}_{p}=\left(\sum_{n=1}^{\infty} \oplus X_{n}\right)_{p}, 1<p<\infty$. Since $W$ is an a-thin set the diagonal space $\Delta \tilde{X}_{p}$ is $\ell_{p}(\mathbb{N})$-saturated $([\mathbb{N}])$. Also $B^{*}$ is isomorphic to a subspace of $\Delta \tilde{X}_{p}^{*}$. Therefore $B$ is a quotient of the space $\Delta \tilde{X}_{p}$ and the proof is complete. Similarly the diagonal space $\Delta \tilde{X}_{0}$ of $\tilde{X}_{0}=\left(\sum_{n=1}^{\infty} \oplus X_{n}\right)_{0}$ is $c_{0}$-saturated and $B^{*}$ is isomorphic to a subspace of $\Delta \tilde{X}_{0}$.

Remark 8.2. (a) It follows from the above theorem that for every $p, r \in(1, \infty)$, $\ell^{p}(\mathbb{N})$ is a quotient of a reflexive $\ell^{r}(\mathbb{N})$-saturated space or a $c_{0}$-saturated space. The result that $\ell^{2}(\mathbb{N})$ is a quotient of a $c_{0}(\mathbb{N})$-saturated space has been proved with a different method by D. Leung ([Le $)$.

(b) It can also be shown that $L^{p}(\lambda), 1<p<\infty$, Tsirelson space $T$, and Schlumprecht $S$ are also quotients of reflexive $\ell^{p}(\mathbb{N})$-saturated or $c_{0}(\mathbb{N})$-saturated spaces.

\section{ACKNOWLEDGMENTS}

We would like to thank Bernard Maurey for his valuable help which allowed us to make an essential step forward and arrive at the present form of the paper. We also thank Nicole Tomczak-Jaegermann for the discussions we had during her visit at Herakleion and Athens. Her suggestion to use quantities similar to Definition 5.3 was really important to us. Finally, we thank Antonis Manoussakis, Irene Deliyanni and Apostolos Giannopoulos for their help during the preparation of the paper.

\section{REFERENCES}

[AA] D. Alspach and S. A. Argyros. Complexity of weakly null sequences, Dissertationes Mathematicae CCCXXI (1992). MR 93j:46014

[AD1] S. A. Argyros and I. Deliyanni. Banach spaces of the type of Tsirelson, preprint 1992.

[AD2] S. A. Argyros and I. Deliyanni. Examples of asymptotic $\ell_{1}$ Banach spaces, Trans. AMS 349 (1997), 973-995. MR 97f:46021

[ADKM] S. A. Argyros, I. Deliyanni, D. Kutzarova and A. Manoussakis, Modified mixed Tsirelson spaces, J. of Func. Anal. 159 (1998), 43-109. CMP 99:04

[AG] S. A. Argyros and I. Gasparis. Unconditional structures of weakly null sequences (Preprint).

[AMT] S. A. Argyros, S. Merkourakis and A. Tsarpalias. Convex unconditionality and summability of weakly null sequences, Isr. J. Math. 107 (1998), 157-193. MR 99m:46021

[AO] G. Androulakis and E. Odell. Distorting mixed Tsirelson spaces (Preprint).

[B] S. F. Bellenot. Tsirelson superspaces and $\ell_{p}$, Journ. of Funct. Analysis. 69 (1986), No2, 207-228. MR 88f:46033

[Bo1] J. Bourgain. Convergent sequences of continuous functions, Bull. Soc. Math. Belg. Ser. B 32 (1980), 235-249. MR 84e:46018

[Bo2] J. Bourgain. La propriété de Radon-Nicodym, Math. Univ. Pierre et Marie Curie 36 (1979).

[D] J. Diestel. Sequences and series in Banach spaces, Graduate texts in Math. 92, SpringerVerlag, 1984. MR 85i:46020

[DFJP] W. J. Davis, T. Figiel, W. B. Johnson and A. Pelczynski. Factoring Weakly Compact Operators, Journ. of Funct. Analysis 17 (1974), 311-327. MR 50:8010

[F1] V. Ferenczi. Quotient Hereditarily Idecomposable spaces (Preprint).

[F2] V. Ferenczi. A uniformly convex hereditarily indecomposable Banach space, Israel J. Math. 102 (1997), 199-225. MR 98m:46013 
[J] R. C. James. Bases and reflexivity of Banach spaces, Ann. of Math. 52 (1950), 518-527. MR 12:616b

[G] W. T. Gowers. A new dichotomy for Banach spaces, GAFA 6 (1996), 1083-1093. MR 97m:46017

[G1] W. T. Gowers. A Banach space not containing $c_{0}, \ell_{1}$ or a reflexive subspace, Trans. Amer. Math. Soc. 344 (1994), 407-420. MR 94j:46024

[G2] W. T. Gowers. A remark about the Scalar-Plus-Compact Problem, Convex Geometric Analysis 34 (1998), 111-115. MR 99m:46015

[GM] W. T. Gowers and B. Maurey. The unconditional basic sequence problem, Journal of AMS 6 (1993), 851-874. MR 94k:46021

[Gr] A. Grothendieck. Critères de compacité dans les espaces fonctionelles généraux, Amer. J. Math. 74 (1952), 168-186. MR 13:857e

[H] P. Habala. Banach spaces all of whose subspaces fail the Gordon-Lewis property, Math. Ann. 310 (1998), No2, 197-212. CMP 98:07

[Ke] A. Kechris. Classical Descriptive Set Theory, Springer-Verlag, Berlin (1994). MR 96e:03057

[KM] K. Kuratowski and A. Mostowski. Set Theory, Amsterdam (1968). MR 37:5100

$[\mathrm{KW}] \quad$ N. Kalton and A. Wilanski. Tauberian operators on Banach spaces, Proc. A.M.S. 57 (1976), 251-255. MR 57:13555

[L] H. E. Lacey. The Isometric Theory of Classical Banach Spaces, Springer-Verlag, Berlin, 1974. MR 58:12308

[Le] D. H. Leung. On $c_{0}$-saturated spaces, Illinois J. Math. 39 (1995), 15-29. MR 96h:46024

[LT] J. Lindenstrauss and L. Tzafriri. Classical Banach spaces I, Springer Verlag 92, 1977. MR 58:17766

[MR] B. Maurey and H. Rosenthal. Normalized weakly null sequences with no unconditional subsequence, Studia Math. 61 (1977), 77-98. MR 55:11010

[MS] V. Milman and G. Schechtman. Asymptotic Theory of Finite Dimensional Normed Spaces, Lecture Notes in Math. 1200, Springer-Verlag. MR 87m:46038

[N] R. Neidinger. Factoring Operators through hereditarily- $\ell_{p}$ spaces, Lecture Notes in Math. 1166 (1985).

[N1] R. Neidinger. Properties of Tauberian Operators, Dissertation. University of Texas at Austin, 1984.

[NR] R. Neidinger and H. P. Rosenthal. Norm-attainment of linear functional on subspaces and characterizations of Tauberian operators, Pac. J. Math. 118 (1985), 215-228. MR 86f: 46013

[OS] E. Odell and T. Schlumprecht. The distortion problem, Acta Math. 173 (1994), 259-281. MR 96a:46031

[OS1] E. Odell and T. Schlumprecht. A Banach space block finitely universal for monotone bases, Trans. Amer. Math. Soc. (to appear). CMP 98:16

[S] T. Schlumprecht. An arbitrarily distortable Banach space, Israel J. Math 76 (1991), 81-95. MR 93h:46023

[To] N. Tomczak-Jaegermann. Banach spaces of type p have arbitrarily distortable subspaces, GAFA 6 (1996), 1075-1082. MR 98g:46020

[T] A. Tsarpalias. A note on Ramsey property, Proc. A.M.S. 127 (1999), 583-587. MR 99c:04005

[Ts] B. S. Tsirelson. Not every Banach space contains $\ell_{p}$ or $c_{0}$, Funct. Anal. Appl. 8 (1974), 138-141.

Department of Mathematics, University of Athens, Athens, Greece

E-mail address: sargyros@atlas.uoa.gr

Department of Mathematics, University of Athens, Athens, Greece 